ABSTRACT

Title of Thesis:

MODEL-BASED SYSTEMS ENGINEERING APPLIED TO THE TRAJECTORY PLANNING FOR AUTONOMOUS VEHICLES

Siddharth Bansal, Master of Science in Systems Engineering, 2018

Thesis Directed By:

Professor John S. Baras, Institute for Systems Research

Passing maneuver is a complex driving maneuver and it becomes more challenging in oncoming traffic. In this study, a passing scenario with three vehicles is considered where car 1, an Autonomous Vehicle (AV), is moving behind car 2 in the same lane. The third vehicle is part of the oncoming traffic in the adjacent lane. The primary goal is to model and evaluate a measurement-based decision-making strategy for the AV satisfying driving safety constraints. This strategy is based on the optimal control with the objective to performing the passing maneuver safely. To evaluate the efficiency of the decision-making strategy - probability of safely completing passing maneuver, a model of the system was developed considering all three cars as point-masses. Two binary variables, each representing the collaborative nature of the cars 2 and 3, were defined. These variables show if the two vehicles will collaborate with the AV when they find out about its intention to overtake. Lastly, a sensitivity study and trade-off study are done to determine optimal design parameters for AV's measurement system and decision-making strategy. 


\title{
MODEL-BASED SYSTEMS ENGINEERING APPLIED TO THE TRAJECTORY PLANNING FOR AUTONOMOUS VEHICLES
}

\author{
By
}

Siddharth Bansal

Thesis submitted to the Faculty of the Graduate School of the University of Maryland, College Park, in partial fulfillment of the requirements for the degree of

Master of Science

2018

\section{Advisory Committee:}

Professor John S. Baras, Chair/Advisor

Associate Professor Mark Austin

Assistant Professor Mumu Xu 
(C) Copyright by

Siddharth Bansal

2018 


\section{Dedication}

This thesis is dedicated to my grandfather, Dr. Om Parkash Bansal, my parents, Dr. Sanjay Bansal and Renu Bansal, and my sister, Dr. Pallavi Bansal. 


\section{Acknowledgements}

I sincerely thank my advisor Dr. John S. Baras for giving me the opportunity to work on this very interesting research problem. With his technical expertise, insightful and motivating comments, he has continuously pushed me to increase my knowledge throughout my time here and successfully complete this thesis. His limitless energy and dedication to work always inspire me.

I would like to thank Dr. Mark Austin for his technical knowledge and being extremely accommodating. I would like to thank Dr. Mum Xu for agreeing to serve on my committee and for her constructive feedback during the thesis examination. I acknowledge with deep sense of gratitude and most sincere appreciation; invaluable guidance rendered to me by Dr. John MacCarthy.

I extend my warm regards to Kim Edwards for her proficiency in handling all the administrative issues, and the ISR staff for always trying to do their best to help the students with official matters.

I take great pleasure in acknowledging the support given to me by my friends and fellow students - Navya, Fatima, Kanishka, Garvit and Christos. These two years at the University of Maryland, I met and became friend with exceptional people. I would like to thank them for their cooperation, the interesting discussions we had and the free time we spent together.

Last but not the least; I would like to thank my parents and my sister for their unquestioning and tireless support. They have always supported me and taught me the things that matter most in life. 


\section{Table of Contents}

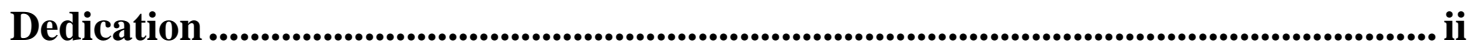

Acknowledgements ............................................................................................. iii

Table of Contents .............................................................................................................. iv

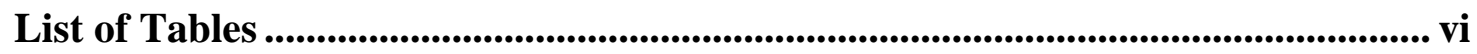

List of Figures............................................................................................. vi

List of Symbols and Abbreviations ....................................................................... viii

Chapter 1: Overview................................................................................................... 1

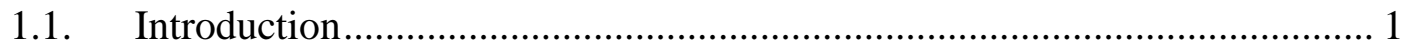

1.2. Relevant Work ...................................................................................... 2

1.3. Problem Statement ................................................................................ 3

1.4. Contribution of Thesis ......................................................................... 5

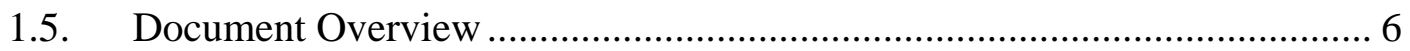

Chapter 2: Car Passing Problem................................................................................ 7

2.1. Problem Background ......................................................................... 7

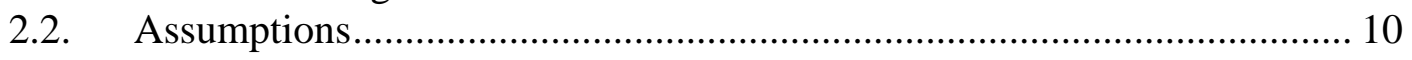

Chapter 3: Model Development and Analysis Approach........................................ 13

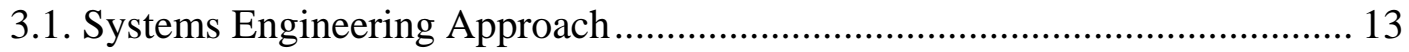

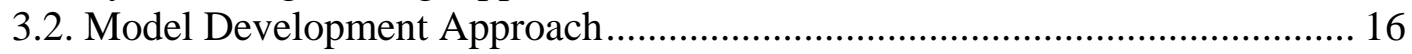

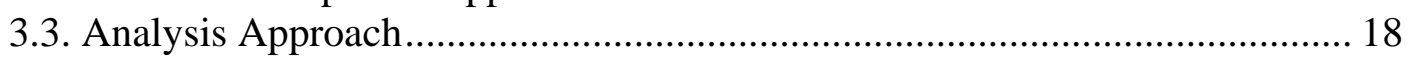

Chapter 4: System Description ........................................................................ 20

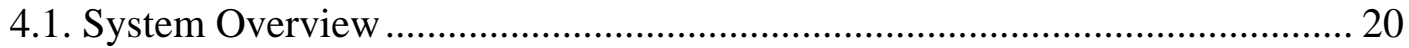

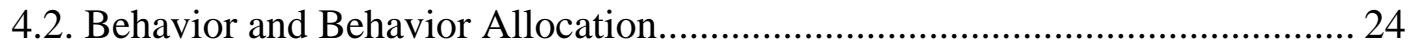

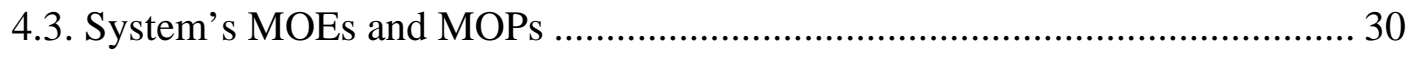

Chapter 5: Car 1 ....................................................................................................... 31

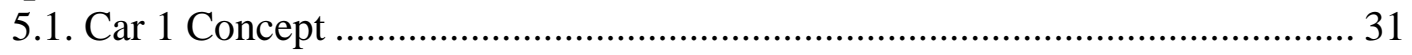

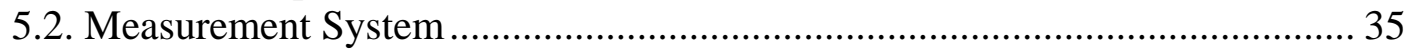

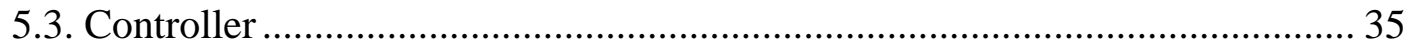

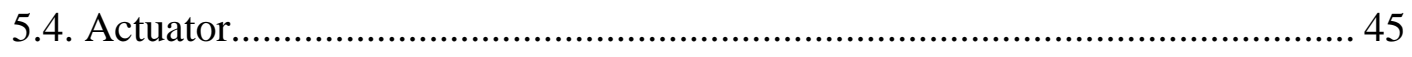

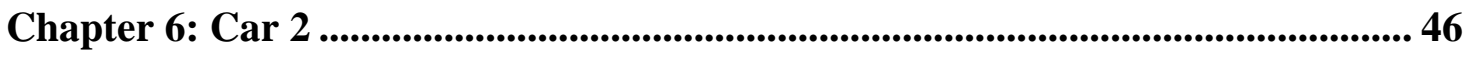

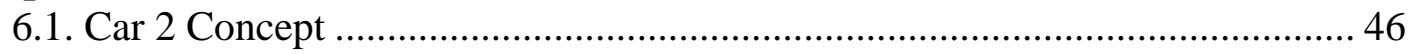

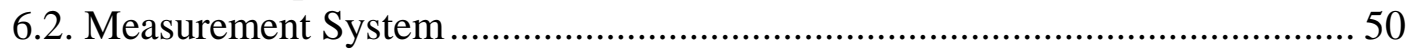

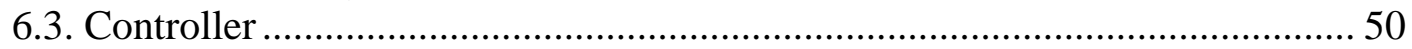

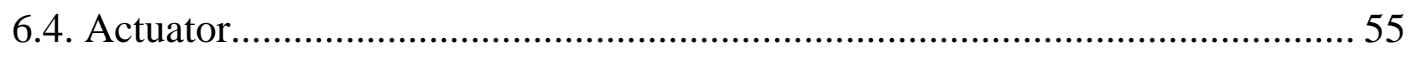

Chapter 7: Car 3 ........................................................................................................ 57

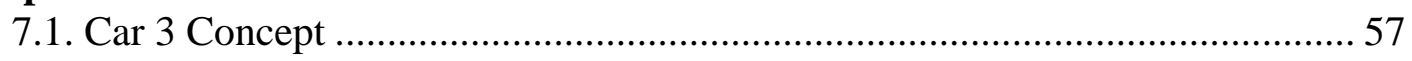

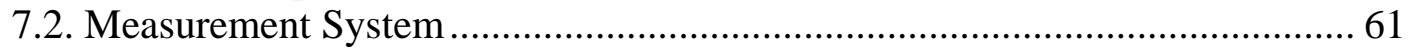

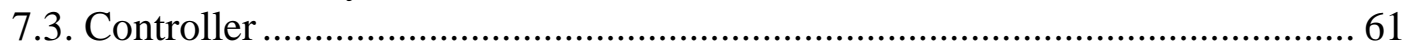

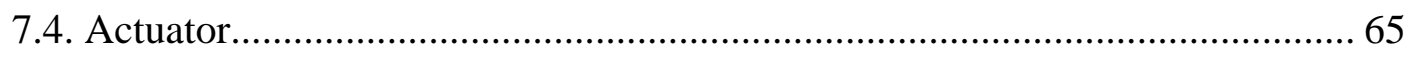

Chapter 8: Simulation ..............................................................................66

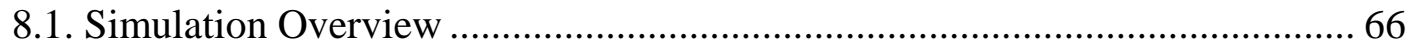

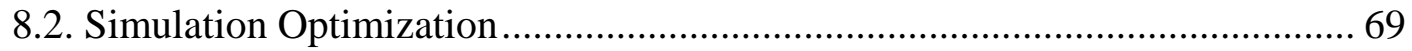

Chapter 9: Analysis, Results and Recommendations ......................................... 73

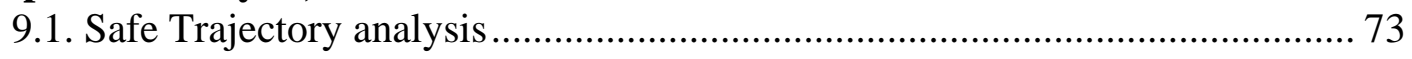

9.2. Simulation Convergence Analysis ........................................................ 74 


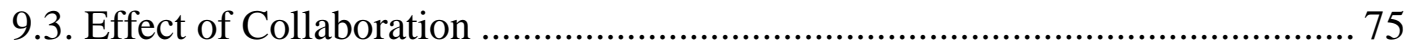

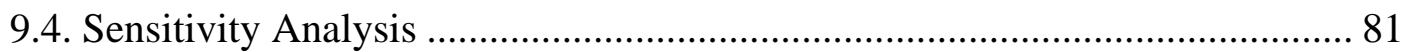

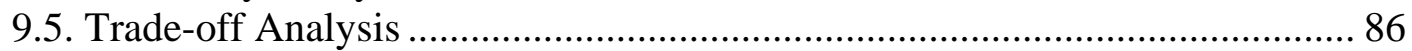

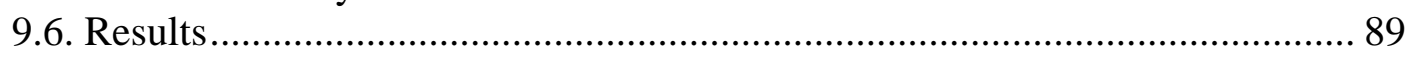

Chapter 10: Conclusions and Future Work ................................................ 91

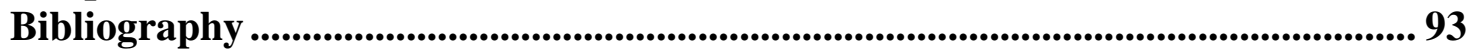




\section{List of Tables}

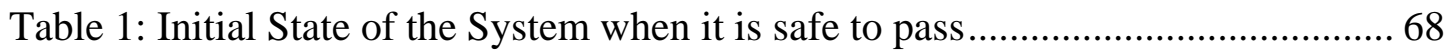

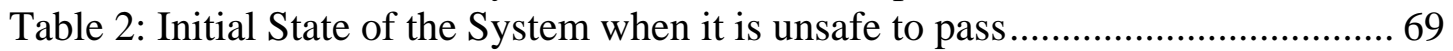

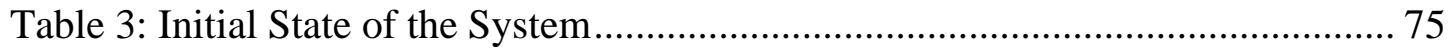

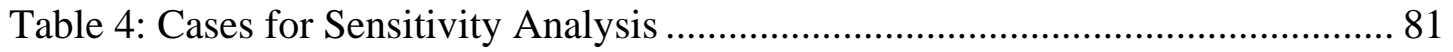

Table 5: Different Design options for Car Passing Maneuver.................................... 87

Table 6: Pareto Dominant Design options for Car Overtaking Maneuver .................. 89

\section{List of Figures}

Figure 1: Problem Scenario ......................................................................... 2

Figure 2: System at $\mathrm{t}=0$ seconds with reference trajectory................................. 8

Figure 3: Response Model Diagram for output metrics showing factors of interest. . 10

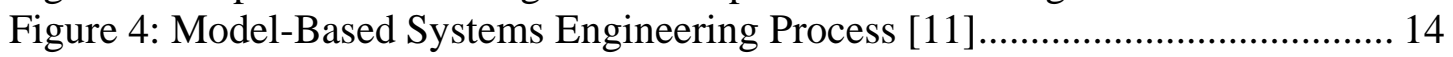

Figure 5: V Development Life Cycle Model (LCM)................................................. 16

Figure 6: Context-level Use Case Diagram for the Car Passing Problem .................. 20

Figure 7: Car Passing Problem Domain Block Definition Diagram........................... 22

Figure 8: Context-level Internal Block Diagram for Car Passing Problem................ 23

Figure 9: Definition of different interfaces shown in context-level IBD.................... 24

Figure 10: Context-level Activity Diagram for Car Passing Problem........................ 28

Figure 11: State Machine diagram for the Car Passing Problem............................... 29

Figure 12: Car 1 Block Definition Diagram .......................................................... 31

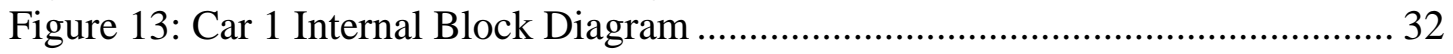

Figure 14: Car 1 Interface Block Definition Diagram ........................................... 33

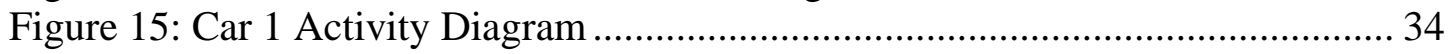

Figure 16: State Machine Diagram for Controller of Car 1.................................... 37

Figure 17: Car 2 Block Definition Diagram ........................................................ 47

Figure 18: Car 2 Interface Block Definition Diagram ........................................... 48

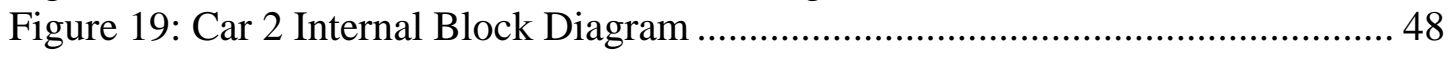

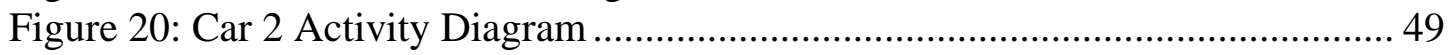

Figure 21: State Machine Diagram for Controller of Car 2.................................... 51

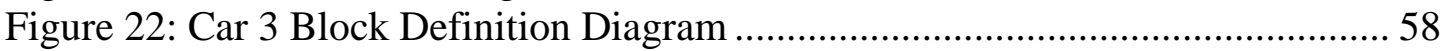

Figure 23: Car 3 Interface Block Definition Diagram ............................................. 59

Figure 24: Car 3 Internal Block Diagram .............................................................. 59

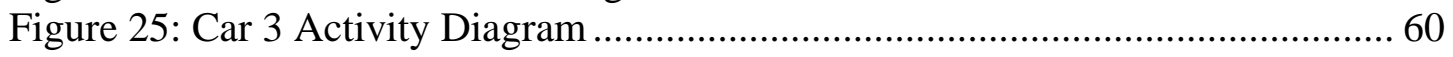

Figure 26: State Machine Diagram for Controller of Car 3........................................ 62

Figure 27: Earlier State Machine Diagram of Car 1 ................................................ 72

Figure 28: Car 1 successfully passing Car 2 without crashing with Car 3 ................ 73

Figure 29: Convergence of $\mathrm{P}(\mathrm{A})$ with increase in iterations.................................... 74

Figure 30: Convergence of $\mathrm{P}(\mathrm{B})$ with increase in iterations .................................. 74

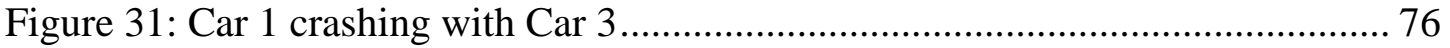

Figure 32: Car 1 passing Car 2 without crashing due to collaboration with Car 2 .... 76

Figure 33: Car 1 successfully passing Car 2 due to collaboration with Car 3 ............ 77

Figure 34: Car 1 passing due to collaboration from Car 2 and Car 3 ....................... 77 
Figure 35: Effect of collaboration and Standard Deviation of Error in Relative

Position measurement (\%) on Safety

Figure 36: Effect of collaboration and Standard Deviation of Error in Relative

Velocity measurement (\%) on Safety 79

Figure 37: Effect of collaboration and Standard Deviation of Error in Relative

Acceleration measurement (\%) on Safety.

Figure 38: Effect of collaboration and Clearance distance of car 1 w.r.t. car 2 at T5 on

Safety

Figure 39: Effect of collaboration and Clearance distance of car 1 w.r.t. car 3 at T5 on

Safety 80

Figure 40: Probability of deciding to pass when it is safe to pass vs Standard deviation of error in Relative Position measurement.....

Figure 41: Probability of deciding to pass when it is safe to pass vs Standard deviation of error in Relative Velocity measurement.

Figure 42: Probability of deciding to pass when it is safe to pass vs Standard

deviation of error in Relative Acceleration measurement

Figure 43: Probability of deciding to pass when it is safe to pass vs clearance distance of car 1 w.r.t. car 2 at T5.

Figure 44: Probability of deciding to pass when it is safe to pass vs clearance distance

of car 1 w.r.t. car 3 at T5. 84

Figure 45: Probability of deciding not to pass when it is unsafe to pass vs Standard Deviation of error in Relative Position Measurement

Figure 46: Probability of safely passing after deciding to pass vs Standard deviation

of error in Relative Position measurement. 85

Figure 47: Probability of deciding not to pass when it is unsafe to pass vs clearance distance of car 1 w.r.t. car 2 at T5. 85

Figure 48: Sensitivity Analysis of $\mathrm{P}(\mathrm{A})$.... 86 


\section{List of Symbols and Abbreviations}

\begin{tabular}{|c|c|}
\hline T0 & $\begin{array}{l}\text { Car } 1 \text { starts to check for passing } \\
\text { feasibility }\end{array}$ \\
\hline $\mathbf{X}_{\mathbf{i}}$ & X position of car ' $\mathrm{i}$ ' \\
\hline $\mathbf{y}_{\mathbf{i}}$ & Y position of car 'i' \\
\hline $\mathbf{a}_{\mathbf{i}}$ & Acceleration position of car ' $\mathrm{i}$ ' \\
\hline $\mathbf{V i}$ & Velocity position of car 'i’ \\
\hline $\mathbf{a}_{1 i}$ & $\begin{array}{l}\text { Acceleration position of car } 1 \text { in } \\
\text { phase 'i' }\end{array}$ \\
\hline dtM1 & $\begin{array}{l}\text { Sampling rate of car } 1 \text { measurement } \\
\text { system }\end{array}$ \\
\hline dtM2 & Reaction time of driver of car 2 \\
\hline dtM3 & Reaction time of driver of car 3 \\
\hline MOP & Measures of Performance \\
\hline$\theta_{1}$ & Angular orientation of Car 1 at T1 \\
\hline $\boldsymbol{\theta}_{2}$ & Angular orientation of Car 1 at T5 \\
\hline sd & $\begin{array}{l}\text { Safe distance for system for checking } \\
\text { collision }\end{array}$ \\
\hline $\mathbf{s d}_{\mathbf{i}}$ & $\begin{array}{l}\text { Safe distance used by car 'i' for } \\
\text { trajectory planning }\end{array}$ \\
\hline $\mathbf{s d}_{125}$ & $\begin{array}{l}\text { Clearance distance of car } 1 \text { w.r.t. car } \\
2 \text { at T5 }\end{array}$ \\
\hline $\mathbf{s d}_{135}$ & $\begin{array}{l}\text { Clearance distance of car } 1 \text { w.r.t. car } \\
3 \text { at T5 }\end{array}$ \\
\hline a1max & Maximum deacceleration of car 1 \\
\hline a2max & Maximum deacceleration of car 2 \\
\hline a3max & Maximum deacceleration of car 3 \\
\hline$d_{1 \max }$ & Maximum deacceleration of car 1 \\
\hline $\mathbf{d}_{2 \max }$ & Maximum deacceleration of car 2 \\
\hline $\mathbf{d}_{3 \max }$ & Maximum deacceleration of car 3 \\
\hline $\mathbf{V}_{\min }$ & Minimum allowed speed on road \\
\hline $\mathbf{V}_{\max }$ & Maximum allowed speed on road \\
\hline MBSE & Model-Based Systems Engineering \\
\hline
\end{tabular}

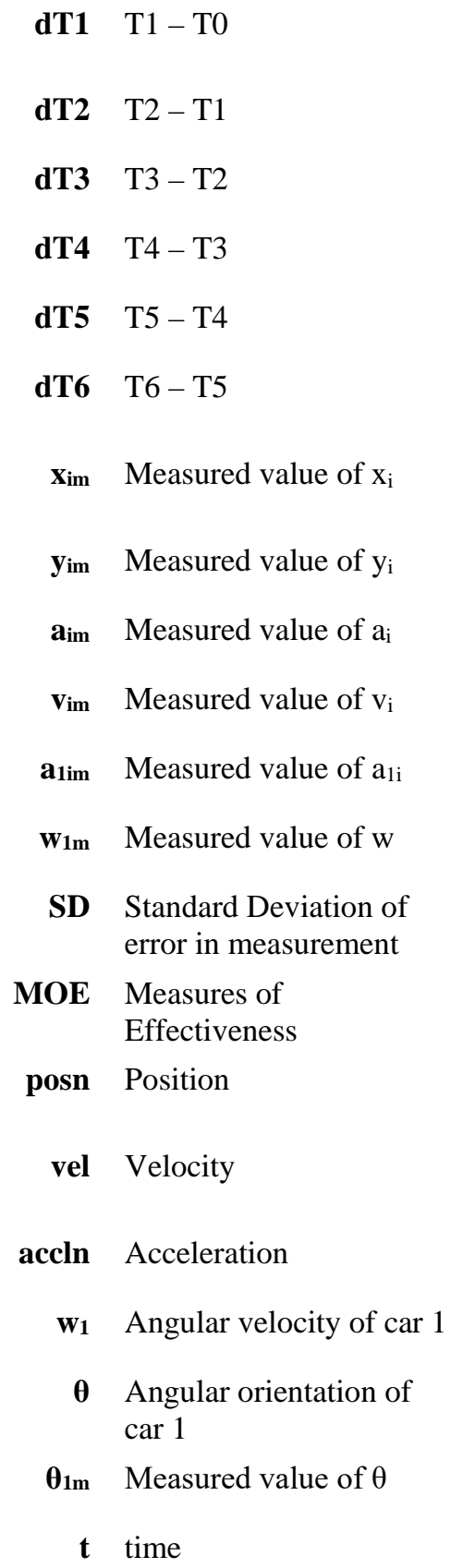
car 1 road 


\section{Chapter 1: Overview}

\subsection{Introduction}

Today, autonomous vehicles are the next big thing in the automotive industry. Principal reason to push forward the autonomous vehicles technology is to address the human element of dynamic driving conditions, like drunken driving and distracted driving as well as solve the problem of traffic congestion. One of the key maneuvers required to solve the problem of traffic congestion is allowing autonomous vehicles to pass other vehicles in traffic. The passing maneuver is especially challenging if other vehicles are driven by humans making the decision to pass prone to elements of human behavior (whether other vehicles collaborate or not), clearance distance between vehicles while turning and accuracy of autonomous vehicle's measurement of the relative dynamics of the other vehicles. All these factors affect AVs decision to pass and thus, traffic flow on the road. For this thesis, passing maneuver refers to a car 1 , the autonomous vehicle, trying to pass a vehicle, car 2, in front of it in presence of oncoming traffic, car 3. Clearance distance is the distance of the car 1 with other vehicles when it is changing lane after passing the other vehicle.

The thesis discusses the effects of behavior of drivers of other cars, error in measurement of relative dynamics of other vehicles, by autonomous car, and clearance distance used to make the decision to pass on the trajectory of the autonomous car. The scenario being addressed in the thesis is shown in Figure 1. 


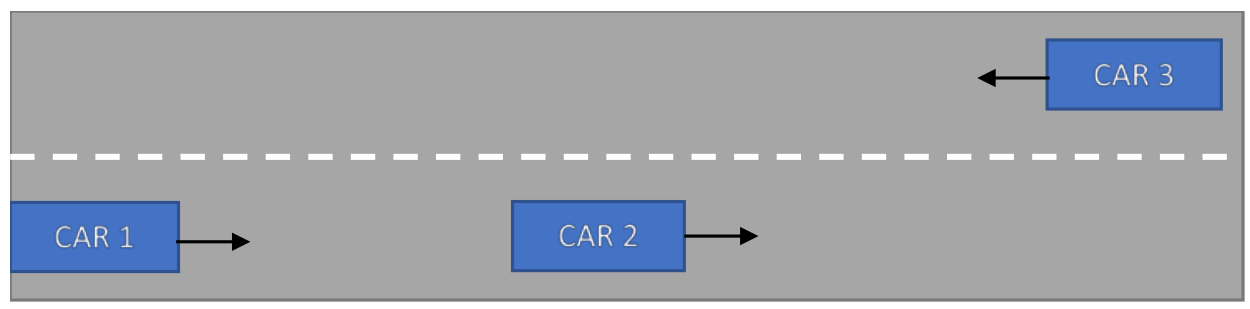

Figure 1: Problem Scenario

\subsection{Relevant Work}

Studies on the passing behavior are focused on a broad variety of objectives like understanding human decision making in passing maneuver, classification of driver behavior, effects of human perception on various aspects of passing, effects of highway design and traffic flow rate on passing maneuver and designing driver assistance systems to aid passing maneuver. From human factors point of view, models have been built to study microscopic gap acceptance models as a function of each driver's perception of the expected time-to-collision (TTC) [1] and Passing maneuver for twoway two-lane rural highways that incorporated the effect of factors such as available sight distance, delay and remaining travel time until the end of the highway segment [2]. Online-capable model-based interaction-aware intention estimation with maneuver-based motion prediction based on supervised learning for dynamic environments has been done [3]. From point of view of highway design and effects of traffic flow on passing maneuver, extensive models have been developed to design and mark criteria for minimum Overtaking zone lengths, with traffic operational efficiency and safety taken into consideration. Work has been done on predicting the over-taking rate/zone in the opposite direction at overtaking zones using traffic and geometric factors [4]. Further, passing maneuver has been extensively studied, which involves regression models to predict the probability to end the passing maneuver with time-to- 
collision (TTC) less than 2 or $3 \mathrm{~s}$ gap from initiation of the maneuver to arrival of the opposite vehicle, and the passing duration as explanatory variables. Highway overtaking scenario with two units have been modelled and simulated using the vehicle simulator 'vehicleSim' which calculated the necessary brake and throttle inputs to match the reference signals [5]. These studies have been used to design a driver assistance system for an overtake maneuver on a highway [6]. Algorithms have been developed that would aid in improving the overall effectiveness of forward collision warning (FCW) systems by providing active safety system designers with further understanding of driver action in overtaking maneuvers [7]. These works have also been incorporated in the upcoming concepts of connected smart cars like evaluation of the effectiveness of a dedicated short-range communication (DSRC)-based wireless vehicle-to- vehicle (V2V) communication system, called the overtaking assistant, devised for improving safety during overtaking (also referred to as passing) maneuvers on two lane rural highways [8].

The work presented here draws inspiration from these works to build a functional architecture that can be used to evaluate the various solutions used to aid the car passing maneuver.

\subsection{Problem Statement}

The primary objective for car 1 is to safely pass car 2 in oncoming traffic while avoiding creating traffic congestion. Safety of cars means that distance between a car and its adjacent cars in the same lane at any time should be greater than the safe distance 'sd'. To achieve this objective, car 1 must first decide to pass and then, execute the 
decision. The decision to pass means finding a feasible trajectory that car 1 can follow to safely execute the passing maneuver. This decision primarily depends on standard deviation of error in relative distance, velocity and acceleration measurements for car 1 and predicted clearance distance of car 1 w.r.t. cars 2 and 3 while executing the passing maneuver.

Based on these parameters, car 1 may or may not decide to execute the passing maneuver. If the decision is to execute the passing maneuver, the correctness of the decision is evaluated by checking the safety of the car 1 while executing the passing maneuver. If the car 1 safely executes the passing maneuver, the decision to pass was correct. From this discussion, the three metrics of interest that can be used to evaluate the performance of the system regarding the primary objective stated in the beginning of this section are - 'probability of deciding to pass given that it is feasible to safely pass' or $\mathrm{P}(\mathrm{A})$, 'probability of deciding not to pass given that it is infeasible to safely pass' or $\mathrm{P}(\mathrm{B})$ and 'probability of safely executing the passing maneuver given that car 1 decides to pass' or $\mathrm{P}(\mathrm{C})$. Based on the objective stated at the beginning of this section, all three probabilities must be minimized.

$P(A)$ is the experimental probability calculated as the ratio of the count of instances when car 1 decided to execute the passing maneuver when it is actually safe to pass versus the count of instances when it is actually safe to pass. $P(B)$ is the experimental probability calculated as the ratio of the count of instances when car 1 decided not to execute the passing maneuver when it is actually unsafe to pass versus the count of instances when it is actually unsafe to pass. $P(C)$ is the experimental 
probability calculated as the ratio of the count of instances when car 1 safely executes the passing maneuver after deciding to pass versus the count of instances when car 1 decides to pass.

\subsection{Contribution of Thesis}

The main contribution of the thesis is the development of a functional architecture of the car passing problem in SysML using Cameo Systems Modeler and using that architecture to structure the problem and build an integrated model to analyze the problem mathematically. Mathematical models of all the components of the problem were coded in MATLAB by the author. The architecture is modular in the sense that the different components can be replaced by higher fidelity models of different design options for that component to analyze system performance because of clearly defined interfaces and abstraction of allocated behaviors.

One of the major contribution of the thesis is that car passing problem has been successfully addressed by showing its dependence on accuracy of measurement system of the vehicle and clearance distance used by the autonomous vehicle to make the decision to pass. To demonstrate this, a mathematical model of different components, including controller for autonomous vehicle, was developed in MATLAB, and performance of the system in terms of making the correct decision whether to pass and safety of the passing maneuver if it is executed, was evaluated.

The use of model of car passing problem to identify best design options for the sensor systems has been demonstrated. The work also shows how MBSE approach can be used in context of car passing problem to facilitate managing complexity, making system modular while allowing design space exploration and evaluation. 


\subsection{Document Overview}

This section provides an outline of the thesis. Chapter 1 starts why the car passing problem should be solved, relevant work and problem statement. This is followed by contribution of the thesis, identification of factors and metrics. Chapter 2 goes into more detail about the problem scenario that is being addressed and relevant assumptions. Chapter 3 the provides details on the model development lifecycle approach and analysis approach. Chapter 4 gives the context-level architecture of the model used to address the car passing problem using structure and behavior diagrams. This is followed by description of structure, behavior, mathematical model and control strategy for different elements of the model - car 1, car 2 and car 3, in chapters 5, 6 and 7. Chapter 8 describes how the model was executed, model parameters, simulation parameters, what data was collected and how. Chapter 9 presents the data collected, results, analysis and recommendation based on the results. Chapter 10 completes the discussion by providing conclusions and possible future work. 


\section{Chapter 2: Car Passing Problem}

This chapter gives details of the problem and explains associated terminologies.

\subsection{Problem Background}

Safety of the trajectory of autonomous vehicle depends on - the initial position and orientation of all vehicles, dynamics of the autonomous vehicle, dynamics of other vehicles, environmental constraints, collaboration amongst the vehicles to support the autonomous vehicle's passing maneuver, clearance distance used by the autonomous vehicle for trajectory planning and errors in information acquired by the vehicles about their dynamics and dynamics of other vehicles. Aim of the experiment is to model the car passing scenario including a controller for the autonomous vehicle that addresses all these factors and demonstrate the model by performing a sensitivity study and tradeoff analyses to maximize safety for the autonomous vehicle in the passing maneuver. As such, Model-based Systems Engineering (MBSE) approach has been used to develop the functional architecture for the problem and analyze its various aspects.

Based on the available information about the dynamics and length of all cars including itself and predicted clearance distance with other cars while turning, controller of car 1 must check the feasibility of the passing maneuver and if the maneuver is feasible, it generates a reference trajectory for the vehicle to follow and safely pass the vehicle in front of it. The reference trajectory is characterized by the acceleration and angular velocity of car 1 during different phases of the passing maneuver, time when car 1 should start to turn to move to the adjacent lane (lane in which car 3 is moving as shown in Figure 2) - referred to as ' $\mathrm{T} 1$ ' in the thesis, and 
orientation of car 1 while turning. To execute passing behavior after determining it is safe to pass other cars, car 1 follows the generic trajectory shown in the Figure 2. For analysis, trajectory of car 1 passing other cars has been divided into six-time intervals or phases, also indicated in Figure 2.

1. T0: Car 1 controller starts checking whether it is safe for car 1 to execute passing maneuver.

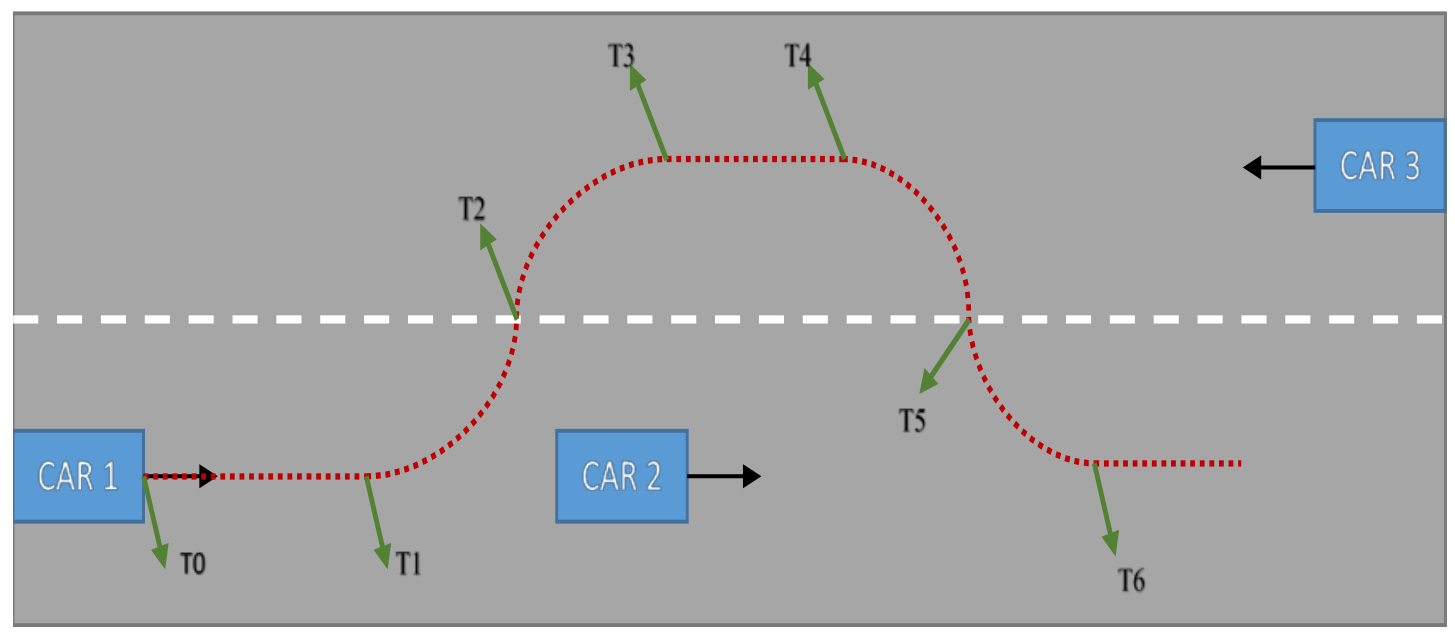

Figure 2: System at $\mathrm{t}=0$ seconds with reference trajectory.

2. T1: Car 1 controller determines it is safe to execute passing maneuver (based on values of measured variables at T1), followed by execution of passing behavior, i.e., car 1 starts turning.

3. T2: Car 1 reaches the end of the lane (white line) and starts entering the other lane.

4. T3: Car 1 finishes turning and starts moving straight in the lane to pass car 2 .

5. T4: Car 1 is at same position along $\mathrm{x}$-axis as car 2 . If car 1 has already crossed car 2 along $\mathrm{x}$-axis by the time $\mathrm{T} 3, \mathrm{~T} 4$ is 0 . 
6. T5: Car 1 reaches the end of the lane (white line) and starts entering the right lane.

7. T6: Car 1 finishes turning and has safely passed car 2.

T0 to T1 is phase 1 with time spent in phase being dT1, T1 to T2 is phase 2 with time spent in phase being dT2, T2 to T3 is phase 3 with time spent in phase being dT3, T3 to T4 is phase 4 with time spent in phase being dT4, T4 to T5 is phase 5 with time spent in phase being dT5 and T5 to T6 is phase 6 with time spent in phase being dT6. The model incorporated collaboration of other cars to support the passing maneuver on T1. The collaboration of the cars is defined in terms of cooperative driver and a neutral driver. A cooperative driver will slow down to support the passing maneuver while a neutral driver will continue at its current velocity. Further, collaboration also depends on at what time do the other cars determine that car 1 is trying to perform passing maneuver and decide to take some action in response.

The objective for car 1 is to safely pass car 2 in oncoming traffic while avoiding creating traffic congestion. To achieve this objective, car 1 must first decide to pass and then, execute the decision. The decision to pass means finding a feasible trajectory that car 1 can follow to safely execute the passing maneuver. This decision primarily depends on the actual initial dynamics and positions of all cars, standard deviation of error in relative distance, velocity and acceleration measurements for car 1, predicted clearance distance of car 1 w.r.t. cars 2 and 3 at T5, length of cars which is reflected in safety distance, maximum allowed speed on road, lane width, assumed angular orientation of car 1's trajectory at T2 ( $\left.\theta_{1}\right)$ and T5 $\left(\theta_{2}\right)$, and maximum acceleration of car 1 . 
Based on these parameters, car 1 may or may not decide to execute the passing maneuver. If car 1 decides against executing passing maneuver, it can slow down traffic flow. The execution of the passing maneuver is characterized by acceleration and angular velocity of car 1 during different phases of the trajectory, angular orientation of car 1 at T2 ( $\left.\theta_{1}\right)$ and T5 ( $\left.\theta_{2}\right)$, and time spent in each phase of the trajectory. These are the control actions of the car 1 . If the car 1 safely executes the passing maneuver, the decision to pass was correct. The response model diagram for the problem is shown in Figure 3.

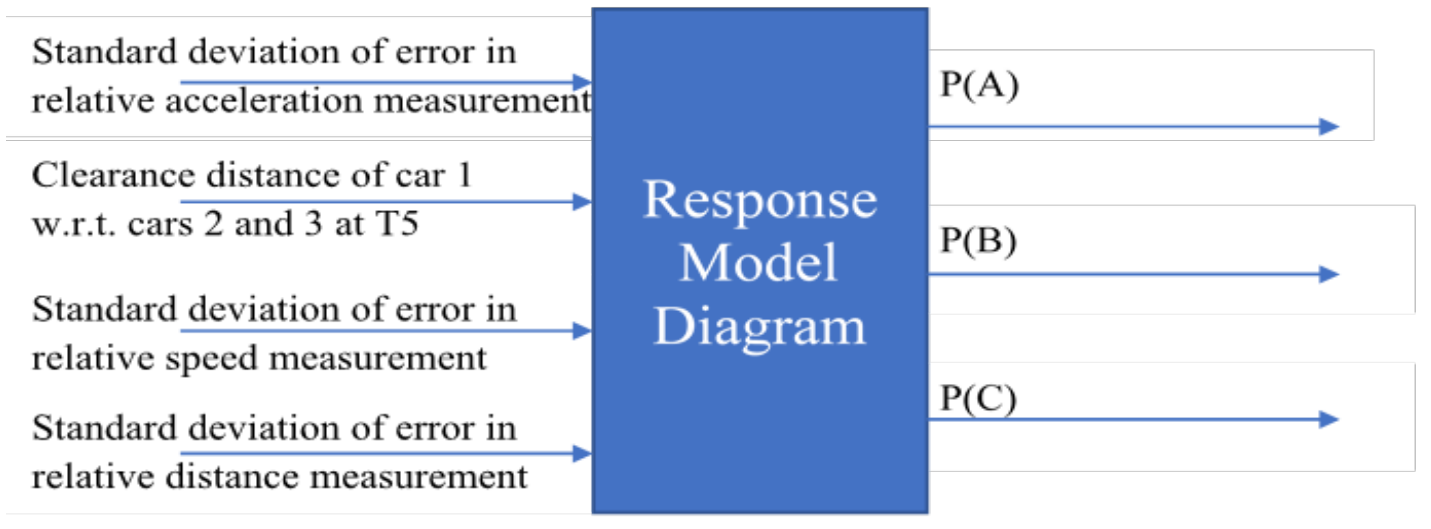

Figure 3: Response Model Diagram for output metrics showing factors of interest.

\subsection{Assumptions}

For the problem defined in previous section there are many assumptions. They are stated below.

- All the cars have been modelled as point masses. The length of the cars is accounted for while considering the safety distance values.

- There is no delay associated with calculation of control action and actuation of control action for car 1. 
- Once car 1 starts executing the passing maneuver, i.e. after time instance T1, it must complete the maneuver. It cannot go back.

- There is no direct communication amongst cars. The information exchange between cars is asynchronous and through sensing.

- All cars must strictly follow the maximum allowed speed limit on road.

- Cars 2 and 3 will start collaborating with car 1 at T3. They may act as cooperative or neutral, as defined towards the end of section 2.1 .

- At T0, it is assumed that car 1 is at the beginning of the road section, car 2 is moving in front of car 1 (both moving towards right and in the same lane) and car 3 is at an x-position near the end of the road section (moving towards left in the adjacent lane).

- At T0, it is assumed that the y-position of car 1 and car 2 is in the middle of the lane they are in and the y-position of car 3 is the middle of the adjacent lane. The y-positions of cars 2 and 3 do not change throughout the simulation.

- From T0 to T3, acceleration of cars 2 and 3 is $0 \mathrm{~m} / \mathrm{s}^{2}$ as cars generally move at relatively constant velocities on a road in absence of any disturbance from the environment. Also, the behavior of interest for cars 2 and 3 is when they start collaborating with car 1 . So, it is a reasonable assumption.

- For car 2 and 3, angular position and angular velocities are assumed to be zero for all the phases of the trajectory.

- From T1 to T3, angular orientation of car 1 is positive and from T4 to T5, angular orientation of car 1 is negative. 
- For phases 2 and 6 of the trajectory, angular velocity of car 1 is positive. For phases 3 and 5 of the trajectory, angular velocity of car 1 is negative.

- At context-level, information exchange between 3 cars is asynchronous. So, simulation is a discrete-event simulation consisting of three parallel simulations of car 1, car 2 and 3 with each simulation being a discrete-time simulation with different sampling frequency.

- Control flow among different components of a car is sequential.

- At the beginning of each simulation run, speed of car 2 must be less than the speed of car 1 so that passing behavior sounds reasonable. 


\section{Chapter 3: Model Development and Analysis Approach}

This chapter gives outlines the systems engineering approach including the development life cycle used to solve the car passing problem. Model development and analysis approach are also discussed in this chapter.

\subsection{Systems Engineering Approach}

Systems Engineering is an interdisciplinary holistic approach to deal with complex systems throughout their lifecycle [9], by establishing the right processes to handle risk and allow the development of a system on time, and on budget while meeting the stakeholders' needs. These processes start from the conceptual phase of the system and continue until its disposal. The car passing problem is critical to road safety, so, a formal system engineering approach - Model-Based Systems Engineering (MBSE) and technical activities have been used.

"Model-Based Systems Engineering (MBSE) is the formalized application of modeling to support system requirements, design, analysis, verification and validation activities beginning with the conceptual design phase and continuing throughout development and later life cycle phases” [10]. MBSE ensures consistency across the multiple views of the model. Building complex models requires considerable resources. As such, models are developed carefully with right amount of fidelity, depending upon project scope, to meet their purpose. In Figure 4, the core steps of the MBSE process, used to develop a model for the car passing problem, are illustrated. The starting point is the available information. This involved literature survey, assessing the existing models of vehicles for reuse and available data about the car passing problem. 
Afterwards, the initial system requirements were identified and defined together with the desired measures of effectiveness ('MOE'). After the requirements phase, the models of behavior and structure were developed using SysML in Cameo Systems Modeler, followed by mapping the specified behavior to the structure. This was followed by developing mathematical models of the system in MATLAB. This is an iterative process, resulting in generation of derivative requirements which prompt changes to the system specification. After a model with sufficient fidelity was ready, tradeoff analysis phase was used to explore feasible design space and choose the best feasible solution based on the specified measures of effectiveness. The chosen design option is then verified and validated ensuring that all the requirements are satisfied, and that the system meets its goals.

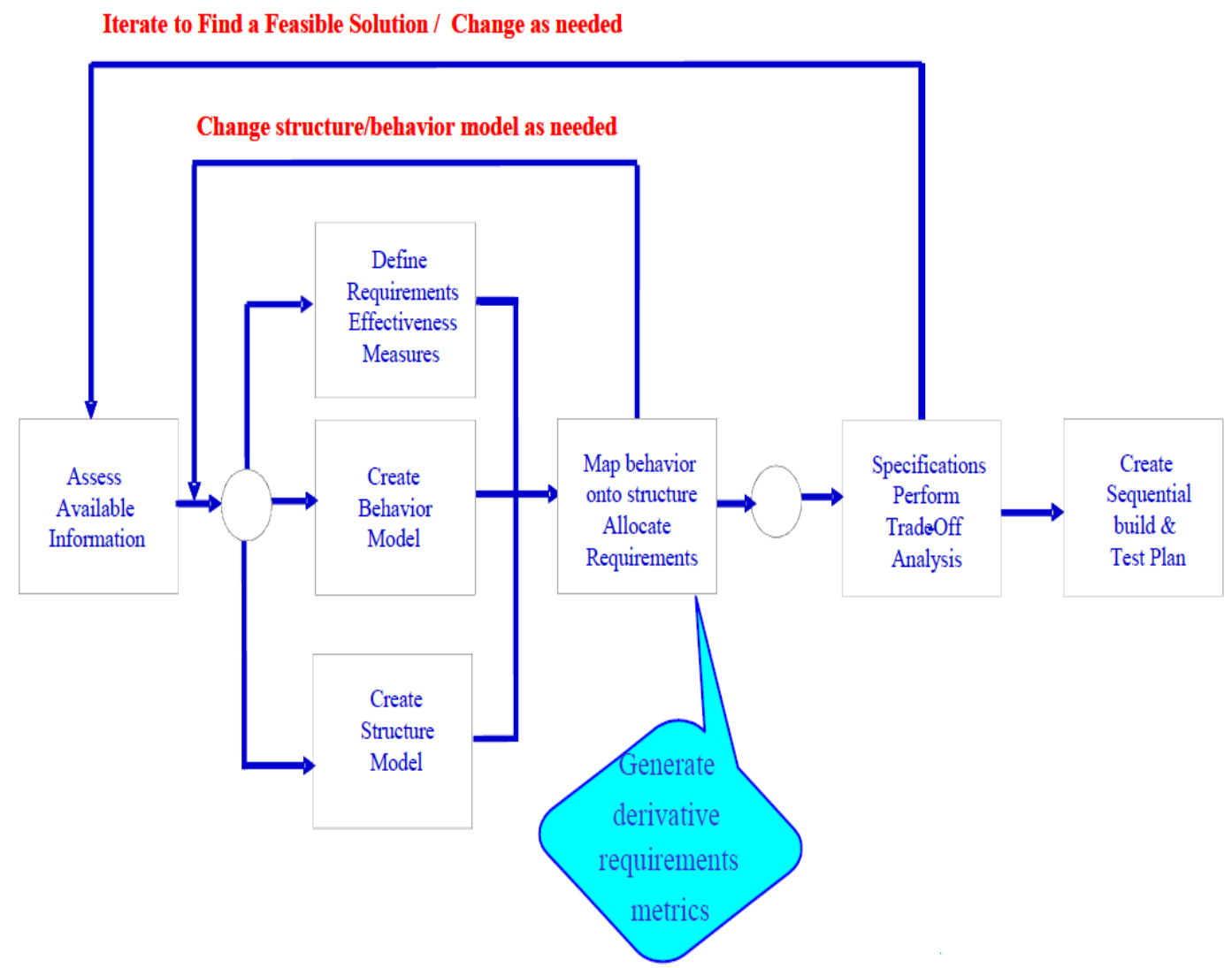

Figure 4: Model-Based Systems Engineering Process [11] 
The greatest advantage of MBSE is the use of models of the system to make decisions and deal with emergent behavior. It ensures fewer errors due to use of formal semantics, consistency across interfaces, drives down costs and reduces time to market while facilitating faster and more rigorous communication between engaged teams and stakeholders, thus, improving overall quality of the system.

The development of model for the car passing problem followed $\mathrm{V}$ Development Life Cycle Model (LCM). The V-model incorporates abstraction and decomposition, the two key concepts in Systems Engineering. At the beginning of the development the user requirements were identified, followed by defining concept of operations for the model, described in Chapter 2. This was followed by iterations of development of model with increasing fidelity and simulation as described in Figure 5. The levels of architecture and requirements will iterate during development life cycle. The level of formality will deepen until a system architecture and requirements definition agrees with the purpose of solving the car passing problem. For this study, model has gone through two complete iterations. At each level of model and simulation development, test plans for verification and validation of the model at that level of model decomposition are developed and updated after each iteration. After this step the right-hand side of the V-model starts. In all those stages mainly, the products of each level are integrated together, while simultaneously the result is checked against the specified requirements. The result is an operational, verified and validated system. In this study, focus is on the left side of the V Development LCM and no test plans have been developed. 


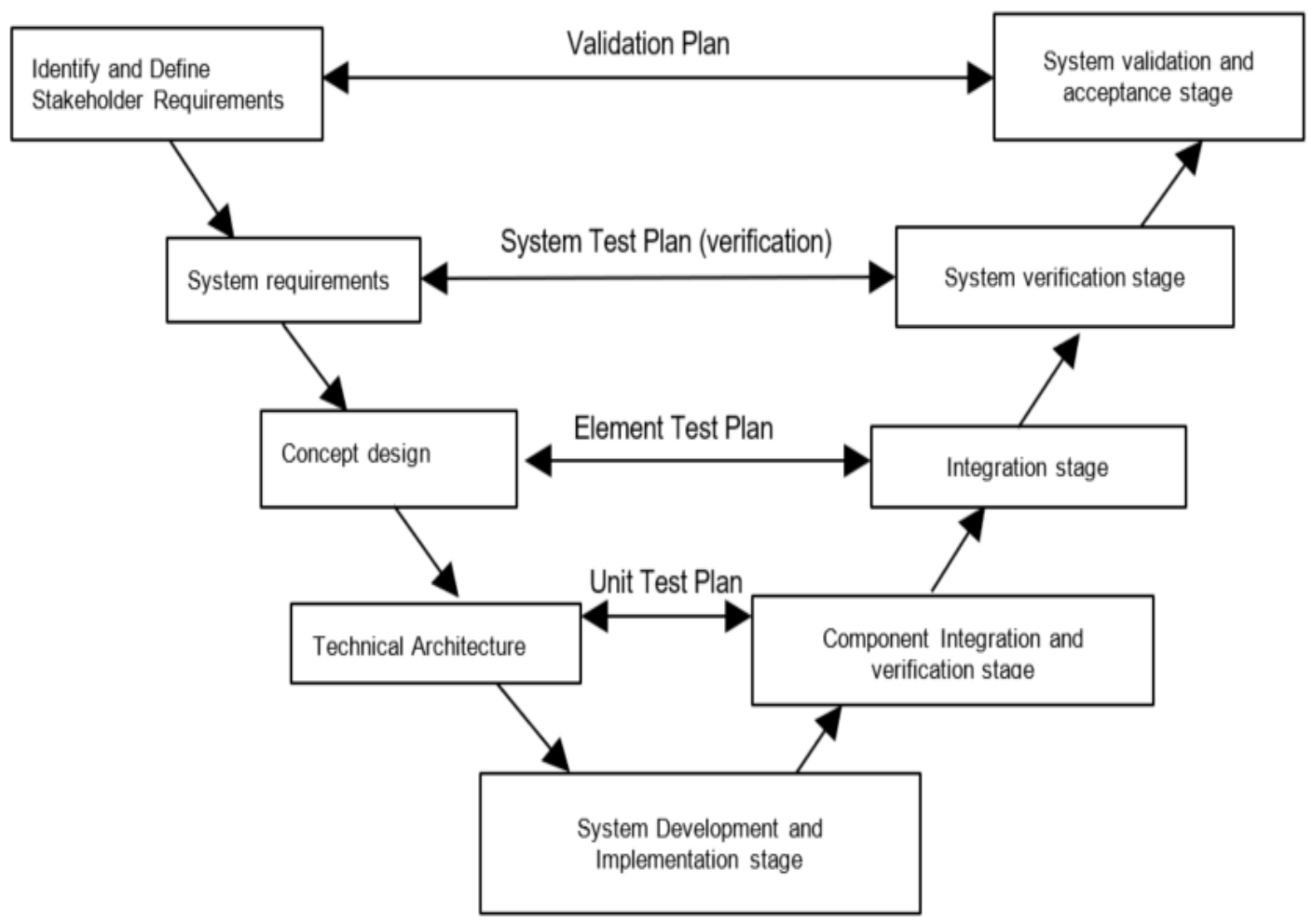

Figure 5: V Development Life Cycle Model (LCM)

\subsection{Model Development Approach}

System has been modelled as a Finite State Machine, defined as a tuple, TS = (S, Act, F, I, AP, Lab) [12] [13]. Cars are point masses. Here,

- $\quad$ ' $\mathrm{S}$ ' is the finite state space characterized by $\mathrm{x} 1, \mathrm{y} 1, \mathrm{x} 2, \mathrm{y} 2, \mathrm{x} 3, \mathrm{y} 3$ and $\theta$.

- 'Act' is the set of actions, i.e., acceleration of cars 1, 2 and 3, and angular velocity of car 1 . These actions act on the present state and determine the next state of the system.

Act $=\left\{\mathrm{a}_{1}, \mathrm{a}_{2}, \mathrm{a}_{3}, \mathrm{w}_{1}\right\}$

- ' $F$ ' is the transition relation or function that calculates the next state based on present state. It uses actions and present state of the system to determine the next state of the system.

$\mathrm{F}: \mathrm{S} \times$ Act $\rightarrow \mathrm{S}$ 
- ' 'I' is the initial state of the system characterized by $\mathrm{v}_{1}, \mathrm{v}_{2}$ and $\mathrm{v}_{3}$ in addition to $\mathrm{x}_{1}$, $\mathrm{y}_{1}, \mathrm{x}_{2}, \mathrm{y}_{2}, \mathrm{x}_{3}, \mathrm{y}_{3}$, and $\theta$.

- 'AP' is the set of atomic propositions (constraints) that characterize each state of the system.

- 'Lab' is the labeling function the state.

$$
\operatorname{Lab}(S)=\left\{\mathrm{x}_{1}, \mathrm{y}_{1}, \mathrm{x}_{2}, \mathrm{y}_{2}, \mathrm{x}_{3}, \mathrm{y}_{3}, \theta\right\}
$$

Using the Finite State Machine defined above, all possible states of the car passing problem have been identified in Figure 11. Using these states, a state machine diagram for car 1 with safe final state is drawn, Figure 16 and further used to define the control logic so that car 1 goes from initial state to safe final state. Safe state refers to when car 1 safely completes the passing maneuver or determines not to execute the passing maneuver. Similarly, state machine diagrams for cars 2 and 3 have been drawn, Figure 21 and Figure 26 respectively and used to develop control logic for the cars. The control logic for all 3 cars was implemented in MATLAB and then, each module was integrated using a 'main' module based on the architecture developed in SysML using the approach defined in section 3.1. This 'CarPassingProblem' module in addition to integrating the car passing model, also collects the data regarding states of the three cars and checks if car 1 is safe or not. The integrated model is simulated using MonteCarlo method to find safe traces of the finite transition system. Safe traces are the traces in which crash does not occur or passing maneuver is not executed. Different trajectories or sequence of states are generated using Monte Carlo method [14] and safety is then checked at each time step of 'dtM1' seconds. 


\subsection{Analysis Approach}

To be able to evaluate the problem a functional architecture of the car passing problem was developed in SysML. This involved development of structure and behavior diagrams, especially state machine diagrams to capture the behaviors of all three cars. The system has been modelled as a Finite State Machine and different traces or trajectories of car 1 have been generated using Monte Carlo method.

For modelling cooperativeness of drivers of cars, a Boolean variable has been defined, where 0 refers to neutral driver and 1 refers to fully cooperative driver. This variable is chosen randomly. Further, car 3 is moving in opposite direction to car $1, V_{3}$ is negative while $V_{1}$ and $V_{2}$ are always positive. The opposite signs of acceleration and velocity of a car indicate deacceleration while same sign indicate acceleration. The mathematical model takes this sign convention into consideration.

Two initial states, one with a feasible reference trajectory and one with infeasible reference trajectory, have been considered. The feasible trajectory is determined by executing the model with $0 \%$ standard deviation of error in measurement for all three cars and keeping clearance distance at nominal values. If the passing maneuver is safely completed by the car 1 , that initial state is recorded for further analysis of the Probability of deciding to pass given that it is feasible to safely pass, $\mathrm{P}(\mathrm{A})$. If the passing maneuver results in crash, that initial state is recorded for further analysis of the Probability deciding to not pass given that it is infeasible to safely pass, P(B). Data recorded for both initial states is used for analysis of the Probability of safely executing the passing maneuver given that car 1 decides to pass, $\mathrm{P}(\mathrm{C})$. 
The first step before proceeding with any analysis was to determine the number of iterations needed for the three probabilities to converge. For each of the two initial states, model is executed several times for different discretized values of clearance distance of car 1 w.r.t. car 2 and car 3 at T5 while keeping standard deviation of error in measurement of distance, velocity and acceleration for car 1 at a fixed reference value. Similarly, then standard deviation of error in measurement of distance is varied, while keeping clearance distance and standard deviation of error in measurement of velocity and acceleration for car 1 at a fixed reference value. Next, standard deviation of error in measurement of velocity is varied, while keeping clearance distance and standard deviation of error in measurement of distance and acceleration for car 1 at a fixed reference value. Lastly standard deviation of error in measurement of acceleration is varied, while keeping clearance distance and standard deviation of error in measurement of velocity and distance for car 1 at a fixed reference value. For each iteration of all the cases, T1, T2, T3, T4, T5, T6, $\theta_{1}, \theta_{2}$, decision of car 1 to execute the passing maneuver and whether maneuver was safely completed or not are recorded. $\mathrm{P}(\mathrm{A}), \mathrm{P}(\mathrm{B})$ and $\mathrm{P}(\mathrm{C})$ are calculated.

$\mathrm{P}(\mathrm{A}), \mathrm{P}(\mathrm{B})$ and $\mathrm{P}(\mathrm{C})$ are calculated for each combination of all the five factors, also called a design point. Pareto analysis is used to define the pareto front and determine the dominating design options. From these reduced set of design options, design option with highest probability of safely executing the passing maneuver given that car 1 decides to pass is chosen. 


\section{Chapter 4: System Description}

This chapter describes the context-level architecture of the car passing problem.

\subsection{System Overview}

The goal of modelling the system is to solve the car passing problem, i.e., car 1 should safely pass car 2 in oncoming traffic. This is the use case, ' $\mathrm{UC1}$ ', for the system as shown in Figure 6. The safety of the car 1 in passing maneuver depends on many environment factors. The focus in this thesis is primarily on dynamics of cars 2 and 3, and road parameters like maximum allowed speed and lane width amongst the environment factors. As such, the primary use case has associations with car 2 (humandriven), car 3 (human-driven) and road. Car passenger uses the car 1 and is the primary user of the use case.

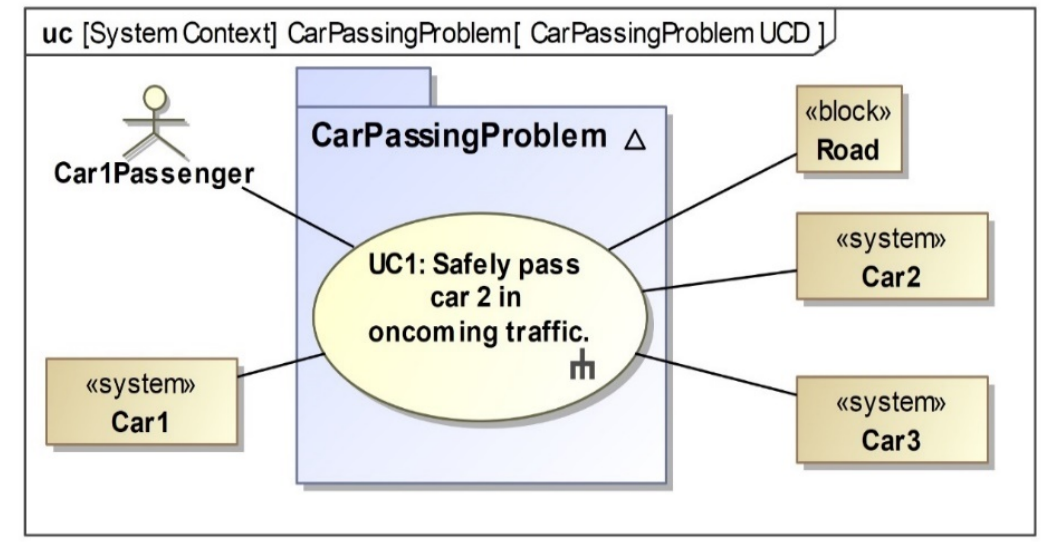

Figure 6: Context-level Use Case Diagram for the Car Passing Problem

Performance of the car 1 regarding the primary use case - UC1 - based on car 1's decision to pass - whether the decision is to execute the passing maneuver or not. I this decision is to execute the passing maneuver, then is the car 1 able to complete the maneuver safely. Thus, the two outputs of interest for the car passing problem are the decision to pass and has the passing maneuver been executed safely. This is shown in 
the ‘CarPassingProblem’ block in the domain block definition diagram shown in Figure 7. Based in the use case diagram in Figure 6, domain of car passing problem consists of car 1 - the system of interest, passenger in car 1 - primary user of the system of interest and environment which interacts with car 1 . In environment, car 1 interacts with car 2, car 3 and road, so, these are contained in the environment block.

The problem itself is characterized by decision of car 1 to pass ('decisionToPass'), whether car 1 completes the passing maneuver safely ('passingCompletedSafely') and the safe distance between the cars that if violated means collision between cars ('sd'). Car 1 is characterized by maximum acceleration (a 1 max) and maximum deacceleration $\left(\mathrm{d}_{1} \max \right)$. Cars 2 and 3 are characterized by maximum acceleration $\left(\mathrm{a}_{2} \max , \mathrm{a}_{3 \max }\right)$ and maximum deacceleration $\left(\mathrm{d}_{2 \max }, \mathrm{d}_{3 \max }\right)$. As cars 2 and 3 do not turn during execution of the passing maneuver, maximum angular velocity for the two cars is not of concern. Further, it is assumed that maximum design speed of all 3 cars exceeds maximum allowed speed on road. This along with the restriction that none of the cars can speed up more than the maximum allowed speed on road, makes information on maximum design speed of cars irrelevant to the problem. The road is characterized by the lane width, minimum allowed speed and maximum allowed speed. The diagram in Figure 7 also shows the parts, behaviors and communication ports for information exchange for all the entities of interest.

The flow of information amongst all 3 cars and road is shown in Figure 8. The parameters from the road are available to all the cars. This shown via the ports on the road block. Information about dynamics of car 1 is available to car 2 and car 3 . Similarly, information about dynamics of car 2 are available to car 1 and car 3, and 
information about dynamics of car 3 are available to car 1 and car 2. The information across interfaces is susceptible to measurement noise. All the information flow with their types and units across different interfaces at the context-level is defined in the interface flow block definition diagram in Figure 9. Interface ‘Car1Parameters’ carries information from car 1 to other cars. Interface 'Car2Parameters' carries information from car 2 to other cars. Interface 'Car3Parameters' carries information from car 3 to other cars.

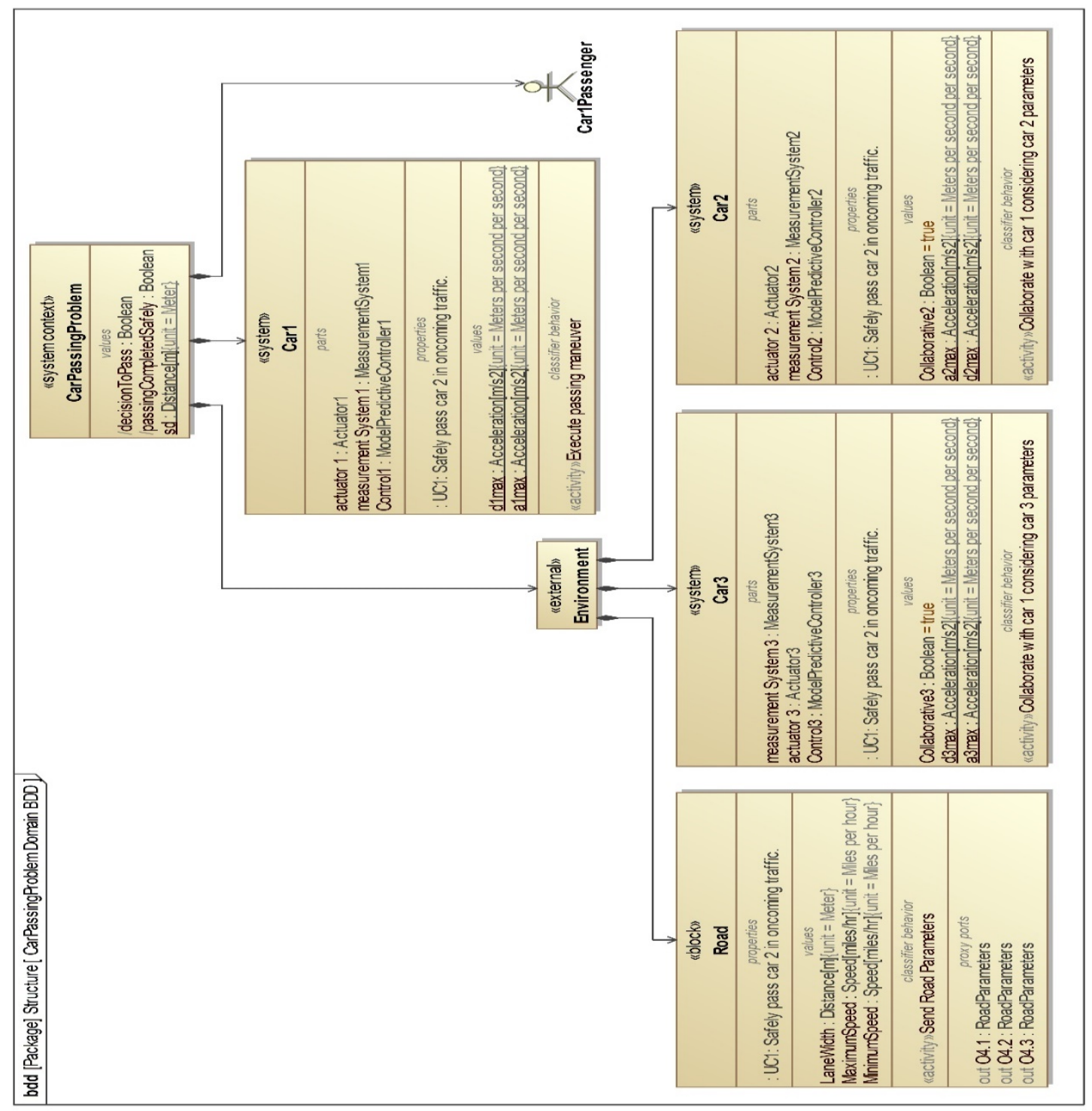

Figure 7: Car Passing Problem Domain Block Definition Diagram. 


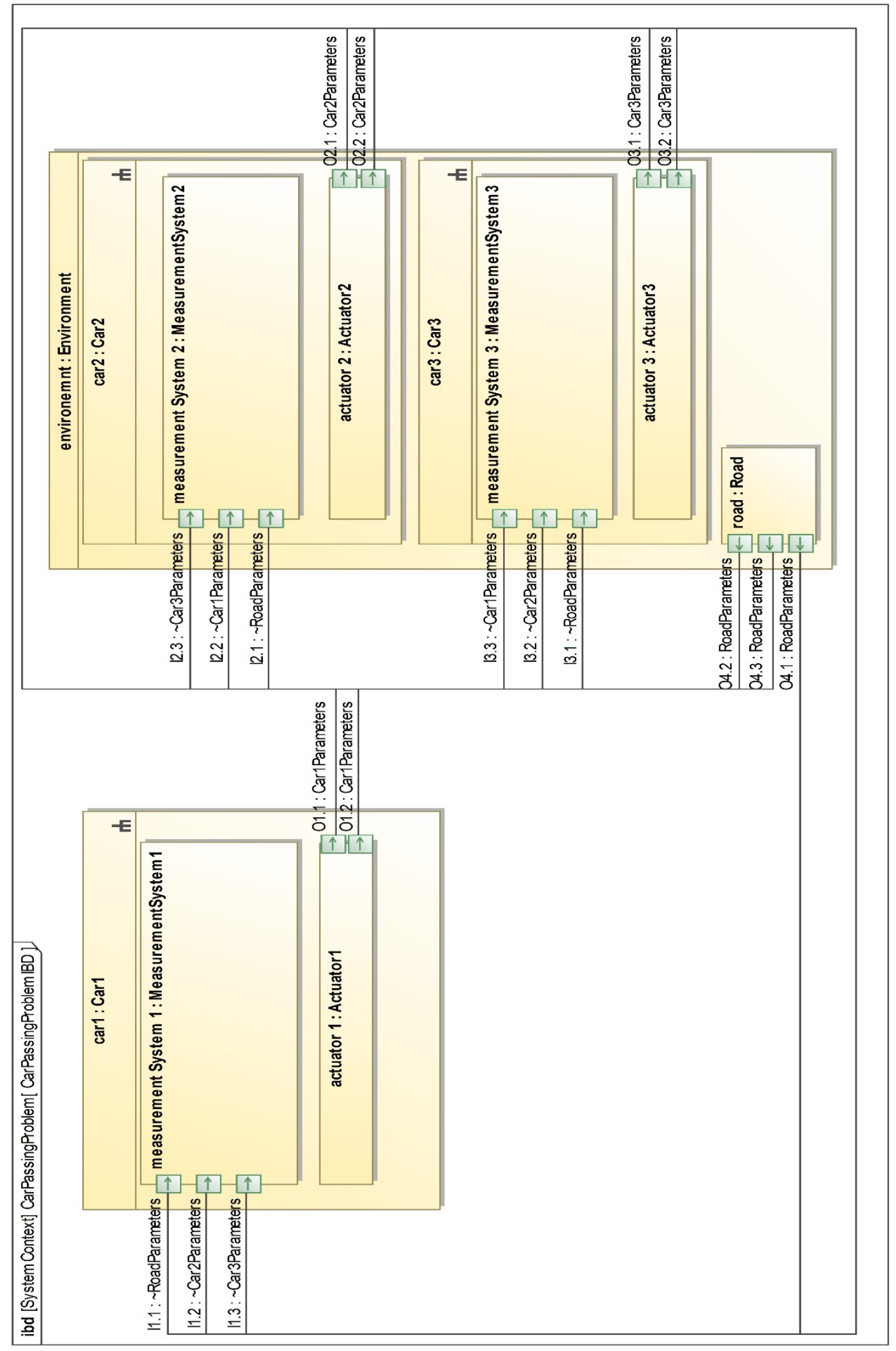

Figure 8: Context-level Internal Block Diagram for Car Passing Problem. 


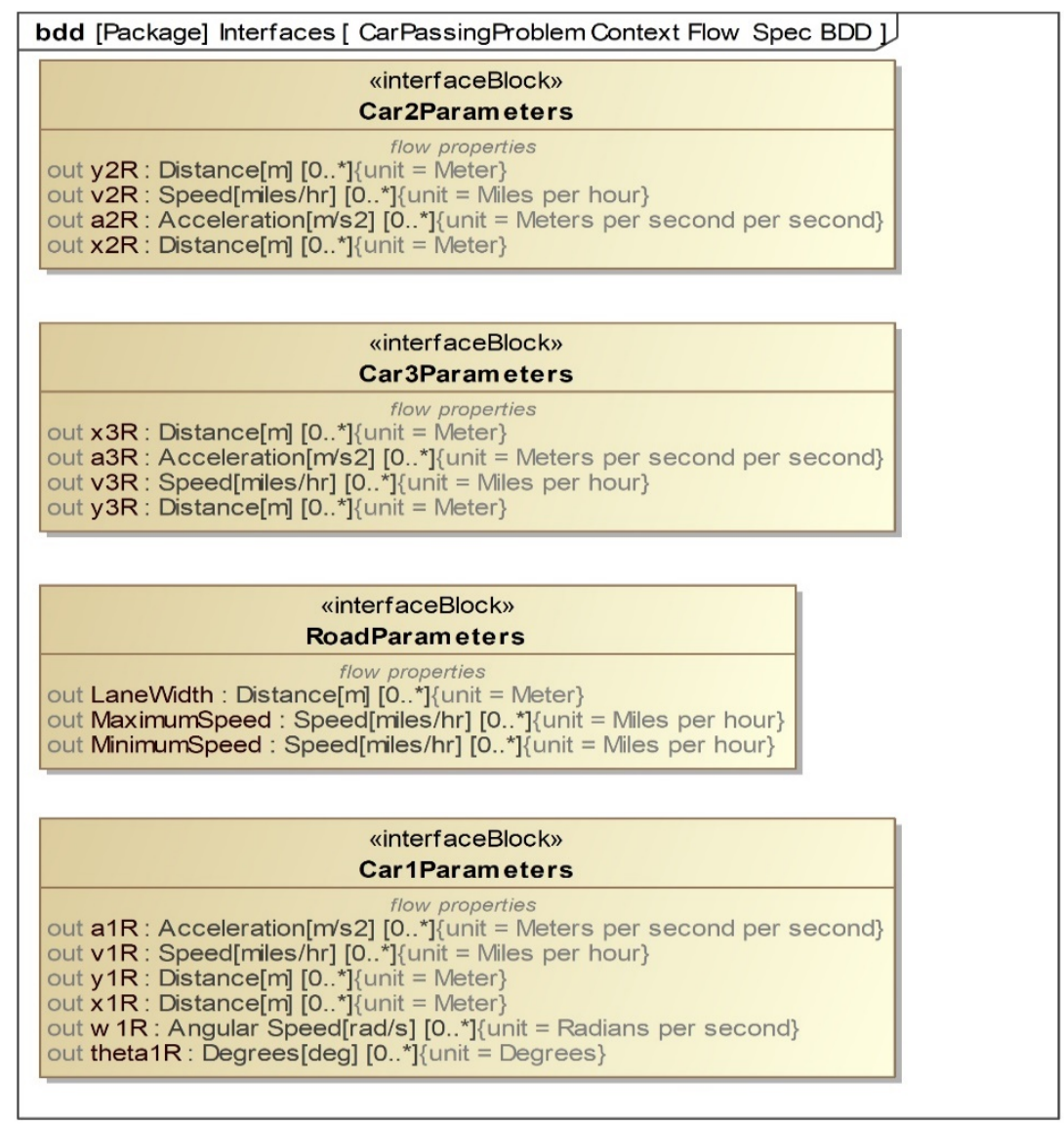

Figure 9: Definition of different interfaces shown in context-level IBD

\subsection{Behavior and Behavior Allocation}

This section defines the interactions among different components of the car passing problems in terms of behavior and how they have been modelled to successfully perform the use case - UC1, using the context-level activity diagram shown in Figure 10. Car 1, car 2, car 3 and road execute the behaviors allocated to them parallelly with information flow between them being asynchronous. As such, there is no starting node on the activity diagram, rather, there are 3 timers with no starting control flow to them. These timers generate a control signal to their respective target behaviors after a fixed time - 'dtM1' for car 1, 'dtM2' for car 2 and 'dtM3' for car 3. There is no synchronization amongst the timers. Road is continuously executing the 
allocated behavior - 'Send Road Parameters'. Solid lines represent flow of data and dashed lines represent flow of control from one behavior to another.

For car 1, after every dtM1 seconds, control flows to the allocated behavior 'Execute passing maneuver'. As soon as control flows to the behavior, it takes the latest information, measurements of cars 1, 2 and 3 as well as road parameters, available at all the input ports and executes the behavior. These ports correspond to the ports on the internal block diagram of car passing problem in Figure 8. Multiplicity of all the input ports is 1 and it is assumed that the latest measurement is available at the input ports. It is assumed that all behaviors are executed instantaneously. After the execution of the behavior, the outputs of the behavior are available at the input ports downstream. Thus, the behaviors are executed sequentially, starting with when control flows to the 'Execute passing maneuver' behavior. Further decomposition of this behavior is provided in chapter 5 . If the passing maneuver is feasible, indicated by the Boolean variable - 'isPassingNotPossible', and not complete, indicated by the Boolean variable - 'isPassingComplete', then the control again goes to the 'Execute passing maneuver' behavior. 'isPassingNotPossible' represent the decision of the car 1 whether to execute the passing maneuver from $\mathrm{T} 0$ to $\mathrm{T} 1$ and it represents whether car 1 crashed from T1 to T6. It is true if the decision is to not pass. i.e., passing maneuver is not feasible or car 1 has crashed. Car 1 has crashed if it satisfies the conditions (4-1) and (4-2) associated with car 1 crashing with car 2 or if it satisfies the conditions (4-3) associated with car 1 crashing with car 3 . The logical 'and' operator between the two conditions is represented using 'join node' in the diagram. If the passing maneuver is not feasible or passing is complete, the control goes to activity final node which marks the end of 
the use case, UC1 and an execution of one instance of the model. The logical 'or' operator between the two conditions is represented using 'decision node' in the diagram.

$$
\begin{aligned}
& \mathrm{x}_{2}(\mathrm{t})-\mathrm{x}_{1}(\mathrm{t})<\mathrm{sd} \text { for } \mathrm{t} \in(\mathrm{T} 1, \mathrm{~T} 2] \\
& \mathrm{x}_{1}(\mathrm{t})-\mathrm{x}_{2}(\mathrm{t})<\mathrm{sd} \text { for } \mathrm{t} \in[\mathrm{T} 5, \mathrm{~T} 6] \\
& \mathrm{x}_{3}(\mathrm{t})-\mathrm{x}_{1}(\mathrm{t})<\text { sd for } \mathrm{t} \in[\mathrm{T} 2, \mathrm{~T} 5] \text { iff } \mathrm{x}_{3}(\mathrm{~T} 2) \geq \mathrm{x}_{1}(\mathrm{~T} 2)
\end{aligned}
$$

For car 2, after every dtM2 seconds, control flows to the allocated behavior 'Collaborate with car 1 considering car 2 parameters'. Here, dtM2 represents the reaction time of the driver of car 2 . As soon as control flows to the behavior, it takes the latest information, measurements of cars 1,2 and 3 as well as road parameters, available at all the input ports and executes the behavior. The behaviors are executed sequentially, starting with when control flows to the 'Collaborate with car 1 considering car 2 parameters' behavior. The behavior uses the available information to decide when to start collaborating and how to collaborate with car 1 in response to the passing maneuver. It is assumed that collaboration starts at T3. Further decomposition of this behavior is provided in chapter 6. Interaction of car 2 with car 1 ends when it determines from the measured information that car 1 is back in the same lane as it and is safely ahead of it, as indicated in the diagram using the condition stated in (4-4) and (4-5). If this condition is satisfied, control goes to flow final node, which marks the end of execution of one instance of car 2's model.

$$
\begin{aligned}
& \mathrm{y}_{1 \mathrm{M}} \leq 0.5 * \text { LaneWidth } \\
& \mathrm{x}_{2 \mathrm{M}}<\mathrm{x}_{1 \mathrm{M}}
\end{aligned}
$$


For car 3, after every dtM3 seconds, control flows to the allocated behavior 'Collaborate with car 1 considering car 3 parameters'. Here, dtM3 represents the reaction time of the driver of car 3. As soon as control flows to the behavior, it takes the latest information, measurements of cars 1, 2 and 3 as well as road parameters, available at all the input ports and executes the behavior. The behaviors are executed sequentially, starting with when control flows to the 'Collaborate with car 1 considering car 3 parameters' behavior. The behavior uses the available information to decide when to start collaborating and how to collaborate with car 1 in response to the passing maneuver. It is assumed that collaboration starts at T3. Further decomposition of this behavior is provided in chapter 7. Interaction of car 3 with car 1 ends when it determines from the measured information that car 1 is not in the same lane as it and is safely ahead of car 2, as indicated in the diagram using the condition stated in (4-6) and (4-7). If this condition is satisfied, control goes to flow final node, which marks the end of execution of one instance of car 3's model.

$\mathrm{y}_{1 \mathrm{M}} \leq$ LaneWidth

$\mathrm{X}_{2 \mathrm{M}}<\mathrm{x}_{1 \mathrm{M}}$

From the perspective of car 1, system has 6 states or phases indicated to in Figure 2 with guard conditions for entry to and exit from a state defined in the state machine diagram in Figure 11. The system goes to initial state at T0. The final state has 3 meaning. First, car 1 determines passing is not possible and does not execute the passing maneuver. Second, car 1 successfully completes the maneuver. Third, car 1 crashes. 


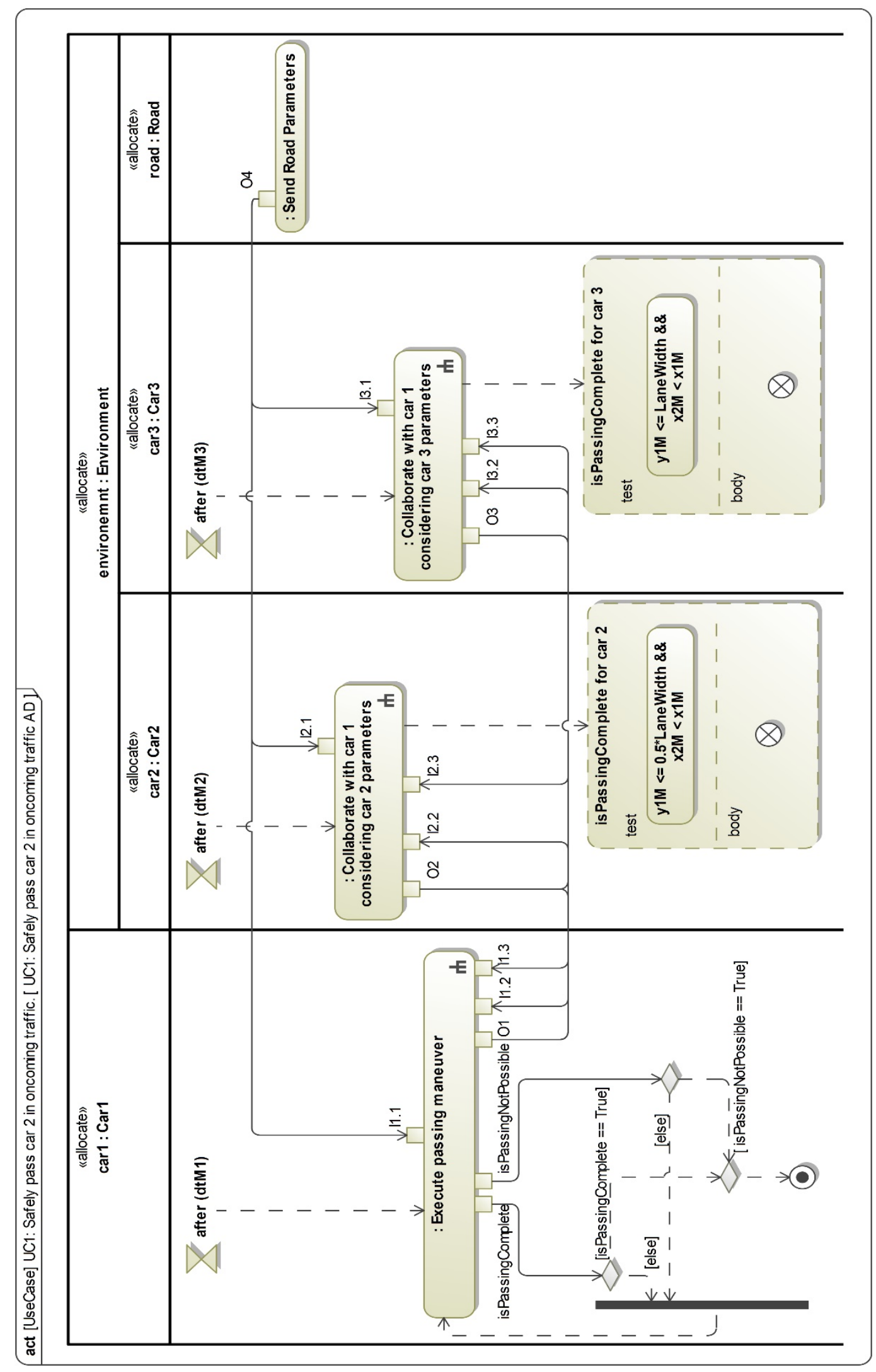

Figure 10: Context-level Activity Diagram for Car Passing Problem. 


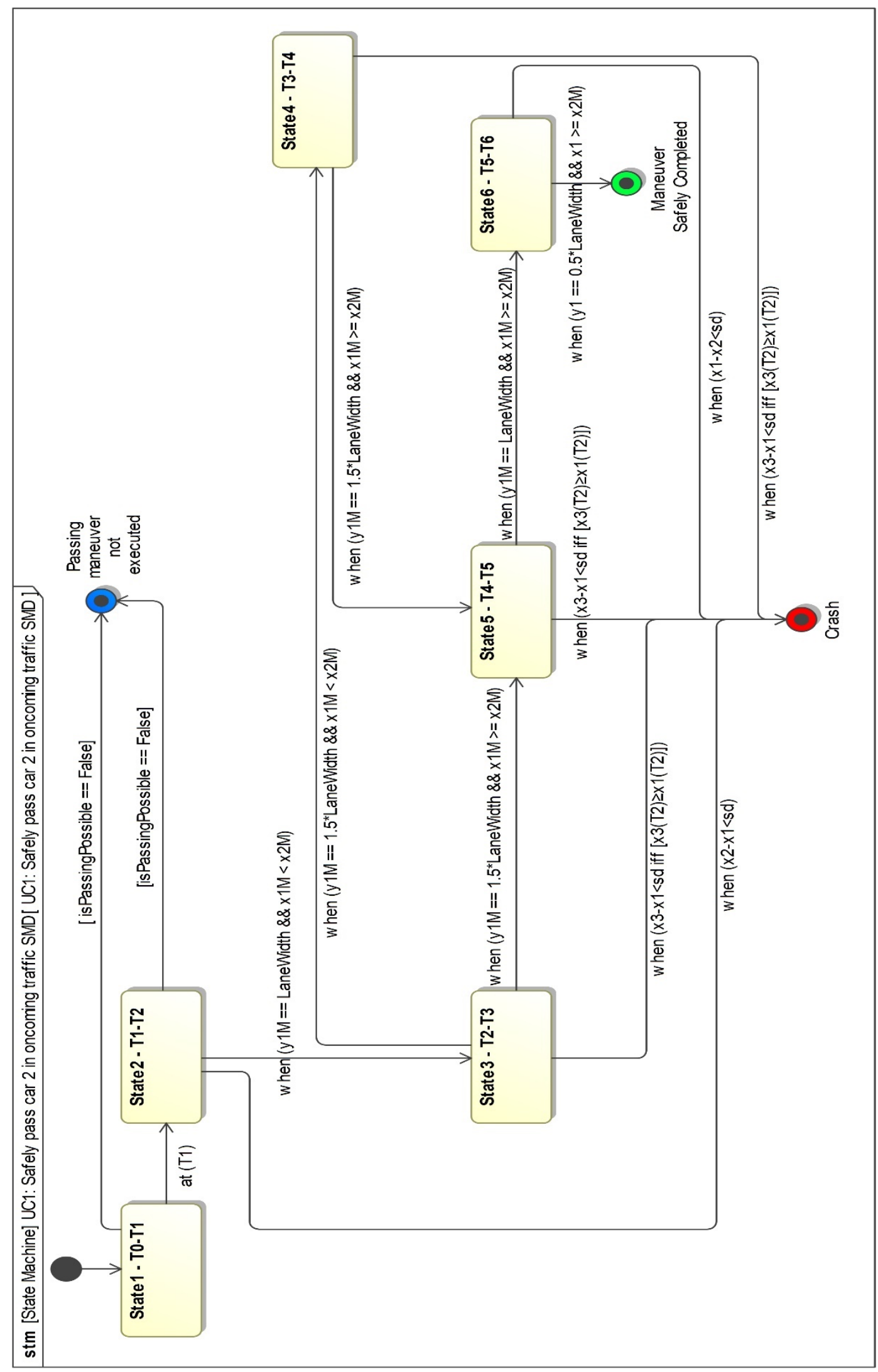

Figure 11: State Machine diagram for the Car Passing Problem 


\subsection{System's MOEs and MOPs}

This section defines the Measures of Effectiveness (MOE) and Measures of Performance (MOP) for the car passing problem. From the discussion in section 1.3 and section 4.1, the parameters of interest are - whether the car 1 makes correct decision to pass and if it makes the decision to pass, can it safely complete the passing maneuver. These are used to evaluate the performance of the car 1's model redefining them as probabilities. Car 1's decision to pass is evaluated using Probability of deciding to pass given that it is feasible to safely pass, $\mathrm{P}(\mathrm{A})$ and Probability of deciding to not pass given that it is infeasible to safely pass, $\mathrm{P}(\mathrm{B})$. If car 1 makes the decision to pass, can it safely complete the passing maneuver is evaluated using the Probability of safely executing the passing maneuver given that car 1 decides to pass, $\mathrm{P}(\mathrm{B}) . \mathrm{P}(\mathrm{A}), \mathrm{P}(\mathrm{B})$ and $\mathrm{P}(\mathrm{C})$ are the MOEs for the system with higher value being indicator of better system performance. Both are calculated over multiple runs of the car passing model, by recording the variables 'decisionToPass' and 'passingCompletedSafely', as defined in Figure 7. These MOEs are directly dependent on standard deviation of error in measurement of relative distance, speed and measurement associated with measurement system of car 1 and clearance distance w.r.t. cars 2 and 3 used to make the passing decision by car 1 , which are the five MOPs of the system. 


\section{Chapter 5: Car 1}

This chapter describes the system-level architecture of the car 1.

\subsection{Car 1 Concept}

Car 1 is the ego vehicle or autonomous vehicle of interest which passes the other car. As outlined in section 4.2 of the thesis, functionality of car 1 is to execute the passing behavior. For execution of the passing behavior, car 1 needs measurements of parameters of all cars and road to determine the control action and then uses the actuator to actuate control action. Thus, car 1 has 3 functional components - 'Measurement System 1', 'Model Predictive Controller 1' and 'Actuator 1', as shown in Figure 12.

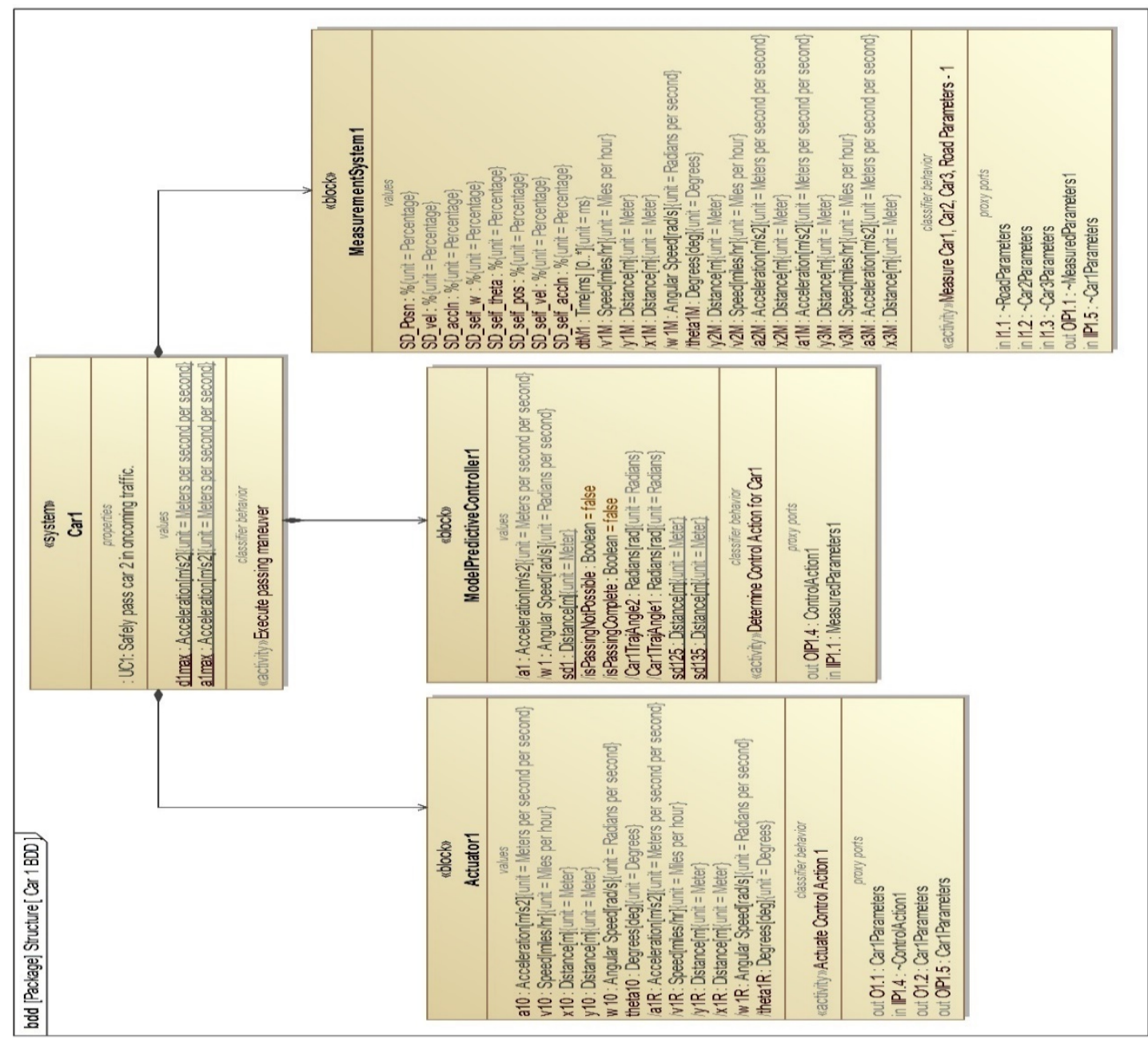

Figure 12: Car 1 Block Definition Diagram 
Measurement system is characterized by standard deviation of error in measurement of car1's position, velocity, acceleration, angular orientation and angular velocity as well as measurements of the relative dynamics of the environment. Standard deviation of error in measurement of dynamics is different for measurements regarding the environment and measurements about car 1 itself. The sampling frequency, denoted by 'dtM1', is also characteristics of measurement system. Model Predictive controller, also referred to as controller, of car 1 is characterized by the clearance distance w.r.t. car 2 at T5 ( $\left.\mathrm{sd}_{125}\right)$ and car 3 at T5 (sd135) used to make the decision to execute the passing behavior, safety distance used to plan the reference trajectory $\left(\operatorname{sd}_{1}\right)$, assumed orientation of car 1 at T2 and T5, decision to pass, variable indicating whether passing is complete and control action, i.e., acceleration and angular velocity of car 1 . The actuator is characterized by the current dynamics of the car 1 and dynamics of car 1 at previous time instant.

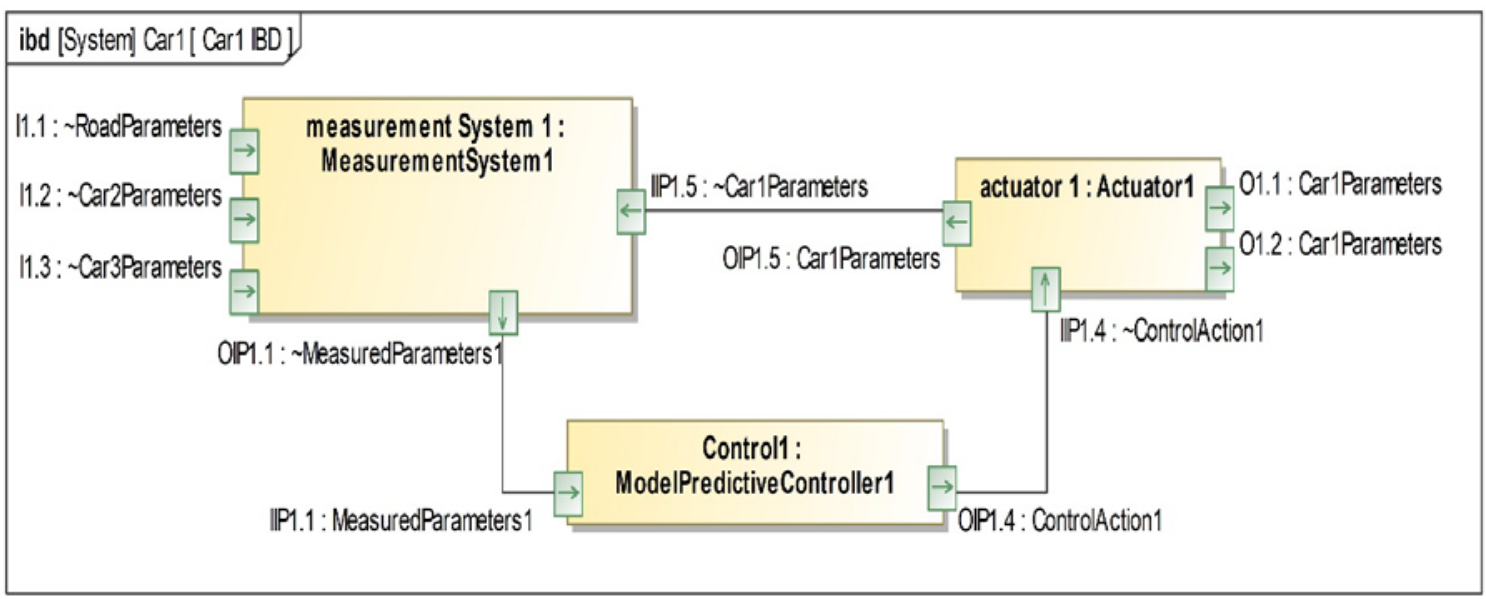

Figure 13: Car 1 Internal Block Diagram

The flow of information amongst different functional components of car 1 is shown the internal block diagram of car 1 in Figure 13. The measurement system collects all the information from the environment and sends it to the controller via 
'MeasuredParameters1' interface. The controller uses that information to generate the control signal and sends it to the actuator via 'ControlAction1' interface. Measurement system measures car 1's parameters. This flow of information occurs via 'Car1Parameters' interface. The data types and values carried in these interfaces is defined in Figure 14 along with the units of each data type.

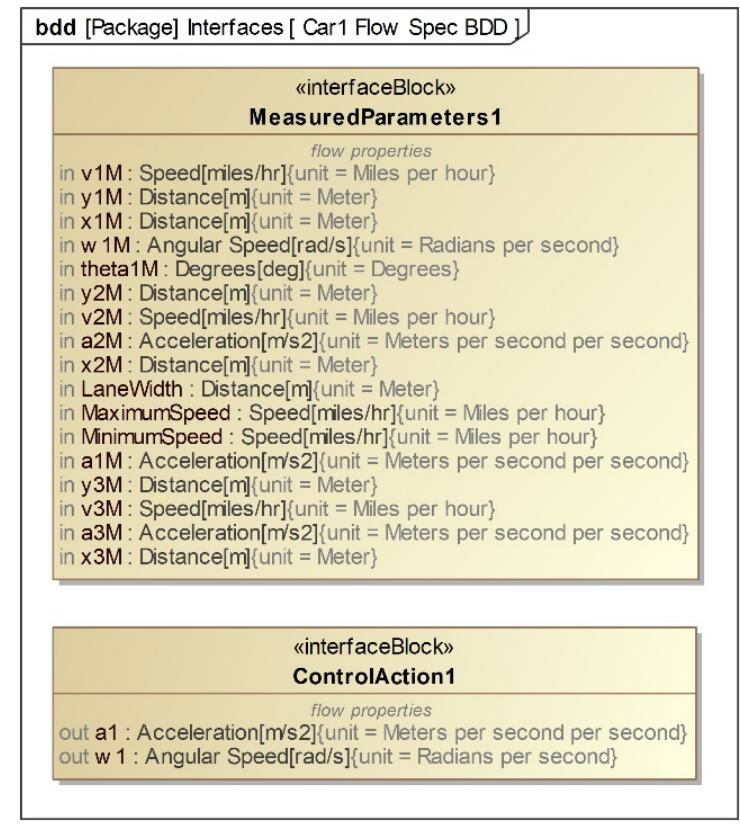

Figure 14: Car 1 Interface Block Definition Diagram

The three functional components of car 1 perform different behaviors. The behaviors allocated to each are shown in Figure 15. This activity diagram is the decomposition of 'Execute passing maneuver' behavior allocated to car 1 . The activity parameters for the 'Execute passing maneuver' behavior, are shown on the diagram frame. Measurement system is allocated behavior - 'Measure car 1, car 2, car 3, road parameters - 1'. Controller executes the behavior - 'Determine control action for car 1'. This behavior outputs the Boolean variables 'isPassingComplete' and 'isPassingNotPossible' to the activity parameters on the diagram's frame. Actuator actuates the control action. The execution of each behavior is determined by the data 
flow. As soon as data is available at all the input ports of a behavior, behavior is executed. This means that multiplicity of all the input ports is 1 . These ports correspond to the ports on the internal block diagram of car 1 in Figure 13. It is assumed that all behaviors are executed instantaneously. After the execution of the behavior, the outputs of the behavior are available at the input ports downstream. Thus, the behaviors are executed sequentially, starting with when data is available to the 'Execute passing maneuver' behavior, i.e., activity parameters on the diagram frame.

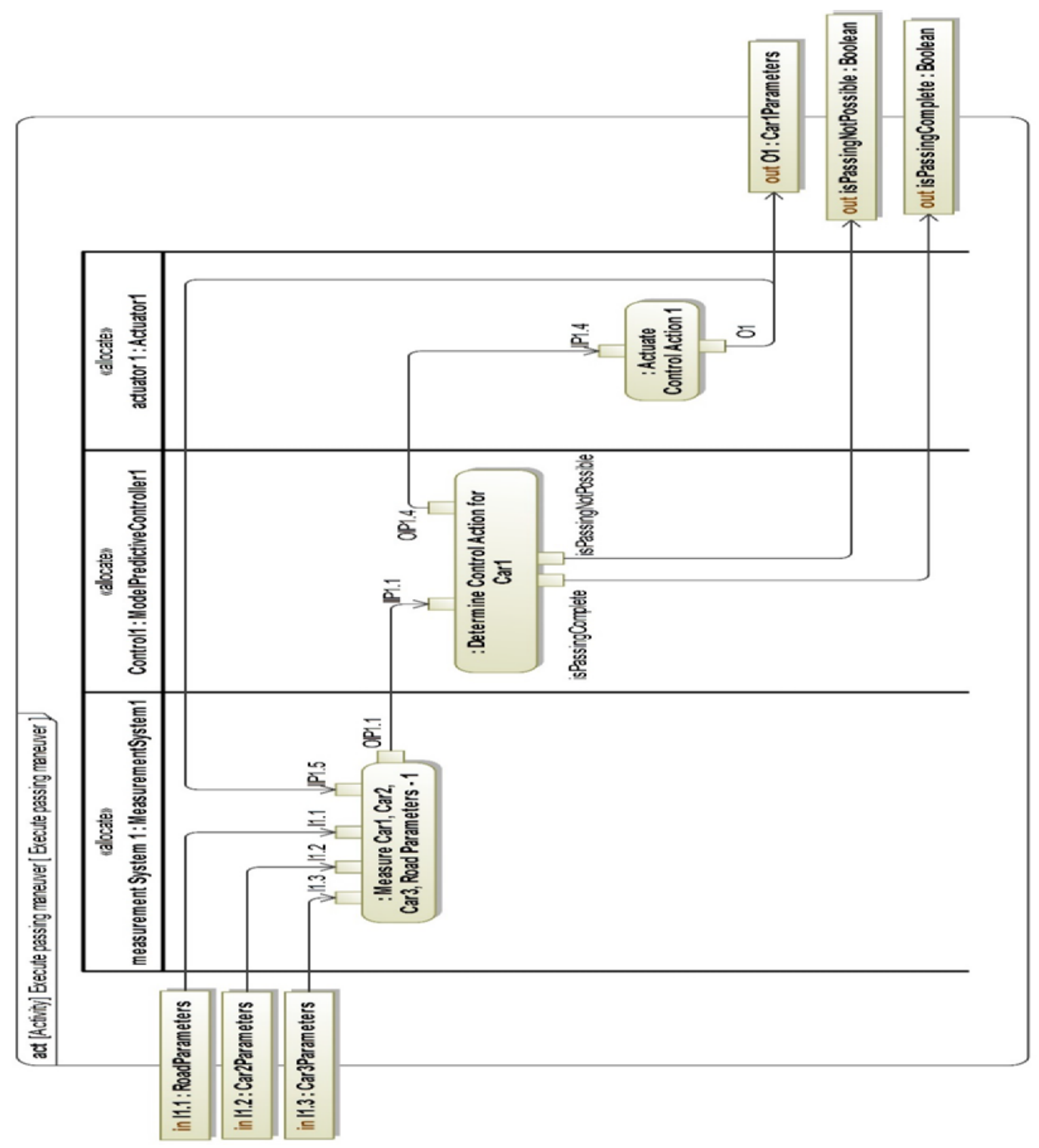

Figure 15: Car 1 Activity Diagram 


\subsection{Measurement System}

The measurement system of car 1 takes measurements of all 3 cars and the road. These measurements are susceptible to measurement noise. As measurement system is a functional element, it represents the overall performance of the measurement system deployed in the car which includes both hardware like different types of sensors, and the software used to decrease the uncertainty in measurement. In the model, this uncertainty in measurements has been modelled as white gaussian noise with zero mean and some standard deviation which is representative of the overall performance of the measurement system. This standard deviation of error in measurement is expressed as percentage of the relative dynamics of other cars and percentage of the absolute dynamics for car 1, denoted by '_self' in the 'Measurement System 1' block in Figure 12. It is assumed to be $0 \%$ for measurements made by car 1 about dynamics of car 1 .

The measurement system measures car 1's position with reference as car 1's position at T0, absolute velocity, absolute acceleration, absolute angular orientation w.r.t. x-axis and absolute angular velocity. It measures relative position, velocity and acceleration of car 2 and car 3 which are then used to calculate their absolute values. It is assumed that measurement information is taken after every dtM1 seconds, sampling rate of car 1, with no lag in information transfer at interfaces associated with the measurement system.

\subsection{Controller}

The function of controller is to determine control action by calculating reference trajectory for car 1 based on measurement data from measurement system of car 1 . The 
objective of controller has been divided in two parts. First part is determining the feasibility of the passing maneuver to decide whether to execute the passing maneuver or not. Feasibility is checked by incorporating clearance distance of car 1 w.r.t. cars 2 and 3 at T5 as constraints. Feasibility of the passing maneuver is first checked at T0. If the maneuver is feasible, controller starts executing the passing maneuver and again checks the feasibility after every dtM1 second till T1. If the maneuver is determined to be unfeasible at more than 2 consecutive time instances from T0 to T1, excluding T1, the passing maneuver is deemed unsafe. If the maneuver is feasible at $\mathrm{T} 1$, controller goes on with executing the passing maneuver. Second part is determining the reference trajectory if the passing maneuver is feasible and update it at the beginning of each phase of the trajectory. This control logic of the controller for different parts of the passing maneuver is shown in the state machine diagram in Figure 16.

The controller has 6 states for 6 phases of passing maneuver trajectory. Each phase of the trajectory has different set of constraints used to determine the reference trajectory. The guard conditions for entry to and exit from a state have been defined in the state machine diagram. The system goes to initial state at $\mathrm{T} 0$ and reaches final state on either successfully completing the passing maneuver or it determines passing maneuver is not feasible. This diagram is based on the context-level state machine diagram presented earlier in section 4.2, Figure 11. It identifies the behavior of the controller in different states as well as the paths to go from initial state to a safe final state, i.e. either passing maneuver is not executed or it is completed safely. But this state control logic susceptible to measurement noise among other factors. The control logic based on this state machine diagram is explained further in this section. 


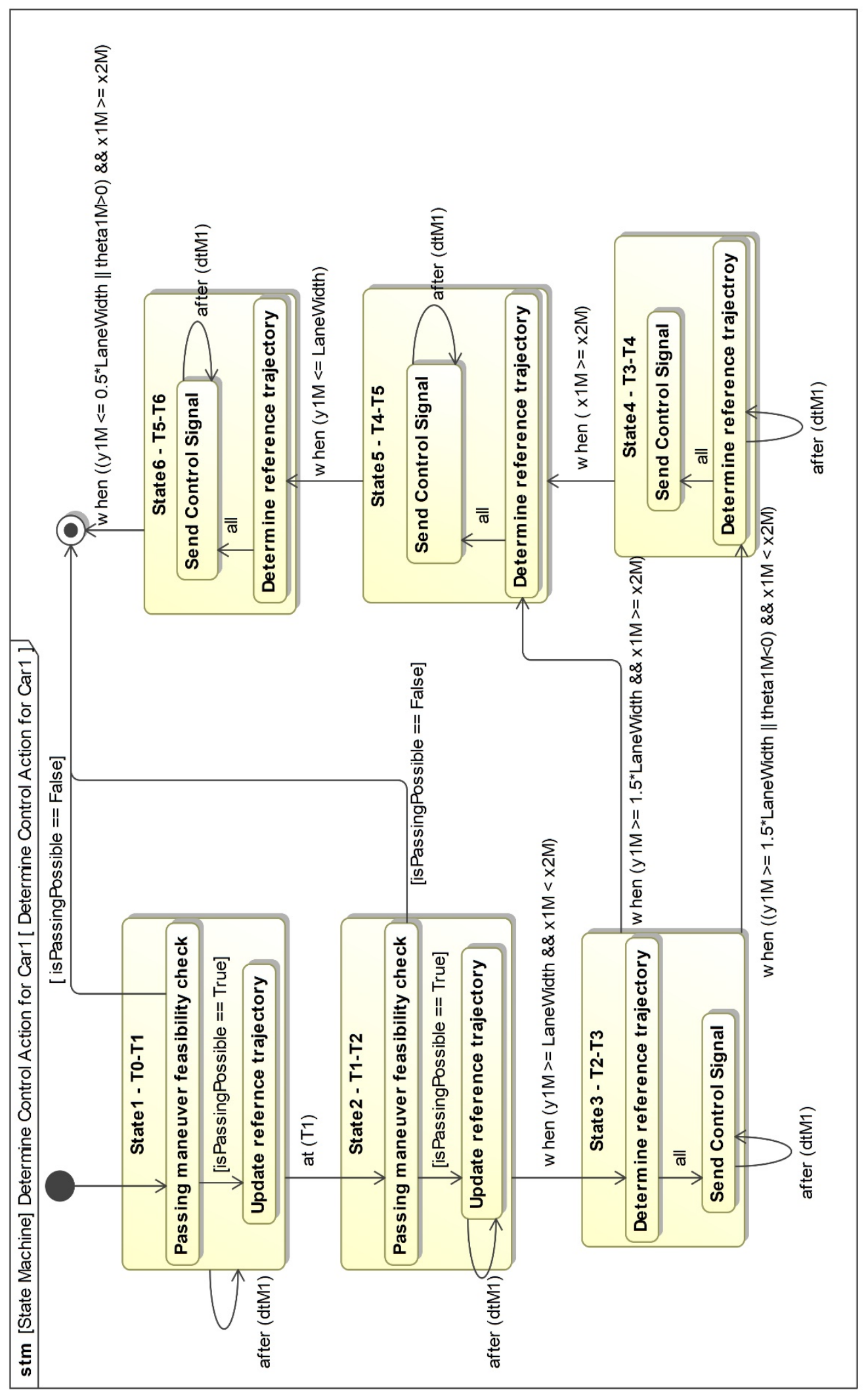

Figure 16: State Machine Diagram for Controller of Car 1 
To safely execute passing maneuver, reference trajectory must satisfy four types of constraints - car safety constraints w.r.t. cars 2 and 3, car 1 specification constraints, road safety constraints and car 1 dynamics constraints. The first 3 types of constraints are inequality constraints and car 1 dynamics constraints are equality constraints. Car safety constraints ensure that car 1 maintains a safe distance of 'sdı' w.r.t. cars in the same lane as car 1 always, as stated below. Safety constraints of car 1 w.r.t. car 2 are:

$$
\begin{aligned}
& \mathrm{x}_{2}\left(\mathrm{~T}_{1}\right)-\mathrm{x}_{1}\left(\mathrm{~T}_{1}\right) \geq \mathrm{sd}_{1} \\
& \mathrm{x}_{2}\left(\mathrm{~T}_{2}\right)-\mathrm{x}_{1}\left(\mathrm{~T}_{2}\right) \geq \mathrm{sd}_{1} \\
& \mathrm{x}_{1}\left(\mathrm{~T}_{5}\right)-\mathrm{x}_{2}\left(\mathrm{~T}_{5}\right) \geq \mathrm{sd}_{1} \\
& \mathrm{x}_{1}\left(\mathrm{~T}_{5}\right)-\mathrm{x}_{2}\left(\mathrm{~T}_{5}\right) \geq \mathrm{sd}_{125} \\
& \mathrm{x}_{1}\left(\mathrm{~T}_{6}\right)-\mathrm{x}_{2}\left(\mathrm{~T}_{6}\right) \geq \mathrm{sd}_{1}
\end{aligned}
$$

Safety constraints of car 1 w.r.t. car 3 are:

$$
\begin{aligned}
& x_{3}\left(T_{3}\right)-x_{1}\left(T_{3}\right) \geq s d_{1} \\
& x_{3}\left(T_{4}\right)-x_{1}\left(T_{4}\right) \geq \operatorname{sd}_{1} \\
& x_{3}\left(T_{5}\right)-x_{1}\left(T_{5}\right) \geq \operatorname{sd}_{1} \\
& x_{3}\left(T_{5}\right)-x_{1}\left(T_{5}\right) \geq s_{135}
\end{aligned}
$$

In the above constraints, if constraint (4-7) is satisfied, it means constraints (45) and (4-6) are satisfied, and are thus, redundant if constraint (4-7) is satisfied. Similarly, if constraint (4-6) is satisfied, constraint (4-5) is redundant. This is because if car 3 is at a safe distance from car 1 at T5 along $\mathrm{x}$-axis, it means it is at a safe distance 
from car 1, always preceding T5. This holds true for car at T4 as well. Thus, if car 3 is at a safe distance from car 1 at T4 along x-axis, it is at a safe distance from car 1, always preceding T4. Road safety constraints ensure that car 1 dynamics are within the speed limits for the road. As such the constraints are stated below.

$\mathrm{v}_{\min } \leq \mathrm{v}_{1}(\mathrm{t}) \leq \mathrm{v}_{\max }, \quad$ where $t \in\left[\mathrm{T}_{0}, \mathrm{~T}_{6}\right]$

Car 1 specification constraints ensure that car 1 dynamics - acceleration and angular velocity - are within the specifications of car 1 . The constraints are stated below.

$0 \leq \mathrm{a}_{1}(\mathrm{t}) \leq \mathrm{a}_{\max }, \quad$ where $\mathrm{t} \in\left[\mathrm{T}_{0}, \mathrm{~T}_{6}\right]$

Constraints (4-1) to (4-9) are all inequality constraints. The car 1 dynamics constraints are based on the definitions of the time instances T2, T3, T4, T5 and T6. They are equality constraints and are stated below.

$$
\begin{aligned}
& \mathrm{y}_{1}\left(\mathrm{~T}_{2}\right)=\mathrm{lw} \\
& \mathrm{y}_{1}\left(\mathrm{~T}_{3}\right)=1.5 * \mathrm{lw} \\
& \mathrm{x}_{1}\left(\mathrm{~T}_{4}\right)=\mathrm{x}_{2}\left(\mathrm{~T}_{4}\right) \\
& \mathrm{y}_{1}\left(\mathrm{~T}_{4}\right)=1.5 * \mathrm{lw} \\
& \mathrm{y}_{1}\left(\mathrm{~T}_{5}\right)=\mathrm{lw} \\
& \mathrm{y}_{1}\left(\mathrm{~T}_{6}\right)=0.5 * \mathrm{lw}
\end{aligned}
$$

At T0, Constraints (4-1), (4-2), (4-3.1), (4-4), (4-7.1) and (4-8) to (4-15) are used to determine the feasibility of the passing maneuver by checking for feasible reference trajectory solutions. The reference trajectory at $\mathrm{T} 0$ refers to the time spent by 
the car 1 in each phase of the trajectory - dT1, dT2, dT3, dT4, dT5, dT6 and the acceleration of car 1 in phase 1 (a11) and phase 4 (a14) of the passing maneuver. It is assumed car 1 does not accelerate in phases 2, 3, 5 and 6. Angular orientation of car 1 at T2 $\left(\theta_{1}\right)$ is fixed to 30 degrees and at T5 $\left(\theta_{2}\right)$ to -30 degrees. For predicting trajectories of cars 2 and 3, car 1 uses measured position, velocity and acceleration of both cars to predict their future trajectories with the assumption that both cars continue with the same acceleration till T3 or till they reach $V_{\max }$ (maximum allowed speed on the road) before T3, after which acceleration of both cars, a2 \& a3, is $0 \mathrm{~m} / \mathrm{s}^{2}$ and that they keep moving in straight line. Under these constraints and conditions, there may be no solutions, one solution or multiple solutions. If there are no solutions, passing maneuver is deemed unsafe and is not executed. Car 1 follows car 2. If there is one solution or multiple solutions, it means there is at least one feasible reference trajectory that car 1 can follow to safely execute passing maneuver. Next step is to determine the reference trajectory that best meets the objectives of the car 1 - safely executing passing maneuver. This is done by defining an objective function to express the goal of the passing maneuver mathematically. The objective can be stated in multiple forms like reducing the overall time to execute the passing maneuver - minimize $\left[\mathrm{T}_{6}\right]$. Another way to state the objective is to maximize the clearance distance between cars 1 and 2 at T5. This objective is mathematically stated in (4-16).

$\max .\left[\mathrm{x}_{1}\left(\mathrm{~T}_{5}\right)-\mathrm{x}_{2}\left(\mathrm{~T}_{5}\right)\right]^{2}$

The objective stated in (4-16) is used to determine the optimal reference trajectory at T0. The objective ensures that maximum distance is maintained between 
cars 2 and 1 at $\mathrm{T} 5$ which further means that car 1 maintains as much distance as possible from car 2 throughout the trajectory while executing the passing maneuver. This also ensures car 1 maintains as much distance as possible from car 2 from T5 to T6 while executing the passing maneuver. Car 1's controller continuously determines the feasibility of passing maneuver, updates and applies the control action, a11, from T0 to $\mathrm{T} 1$ in steps of dtM1 second, where $\mathrm{T} 1=\mathrm{T} 0+\mathrm{dT} 1$ and $\mathrm{dT} 1$ is one of the solutions of the optimization problem. To make the algorithm more robust, if T1 is determined to be dtM1 seconds or less in the future, controller considers that time instance as T1.

At T1, the controller again checks for the feasibility of the passing maneuver. Constraints (4-2), (4-3.1), (4-4), (4-7.1) and (4-8) to (4-15) are used to determine the feasibility of the passing maneuver by checking for feasible reference trajectory solutions. The reference trajectory at T1 refers to the time spent by the car 1 in remaining phases of the trajectory - dT2, dT3, dT4, dT5, dT6, orientation of car 1 at T2 $\left(\theta_{1}\right)$ and the acceleration of car 1 in phase 2 (a12) and phase 4 (a14) of the passing maneuver. It is assumed car 1 does not accelerate in phases 3, 5 and 6. Angular orientation of car 1 at T5 $\left(\theta_{2}\right)$ is fixed to -30 degrees. For predicting trajectories of cars 2 and 3, car 1 uses measured position, velocity and acceleration of both cars at T1 to predict their future trajectories with the assumption that both cars continue with the same acceleration till T3, after which acceleration of both cars, a2 \& a3, is $0 \mathrm{~m} / \mathrm{s}^{2}$ and that they keep moving in straight line. Under these constraints and conditions, there may be no solutions, one solution or multiple solutions. If there are no solutions, passing maneuver is deemed unsafe and is not executed. Car 1 follows car 2. If there is one solution or multiple solutions, it means there is at least one feasible reference 
trajectory that car 1 can follow to safely execute passing maneuver. Next step is to determine the reference trajectory. The objective stated in (4-16) is used to determine the optimal reference trajectory at T1. 'w1' for car 1 from T1 to T2 is determined by dividing $\theta_{1}$ by $\mathrm{dT} 2$, both are the solutions of the optimization problem. Car 1's controller updates the reference trajectory once at T1 and then continuously applies the control action, w1 and a12, from T1 to T2 in steps of dtM1 second.

At T2, the controller updates the reference trajectory. Constraints (4-3) to (44), (4-7) to (4-9) and (4-11) to (4-15) are used to determine the feasible reference trajectory solutions. The reference trajectory at $\mathrm{T} 2$ refers to the time spent by the car 1 in remaining phases of the trajectory - dT3, dT4, dT5, dT6, and the acceleration of car 1 in phase 3 (a13) and phase 4 (a14) of the passing maneuver. It is assumed car 1 does not accelerate in phases 5 and 6 . Angular orientation of car 1 at T5 $\left(\theta_{2}\right)$ is fixed to -30 degrees. For predicting trajectories of cars 2 and 3, car 1 uses measured position, velocity and acceleration of both cars at $\mathrm{T} 2$ to predict their future trajectories with the assumption that both cars continue with the same acceleration till T3, after which acceleration of both cars, a2 \& $\mathrm{a}_{3}$, is $0 \mathrm{~m} / \mathrm{s}^{2}$ and that they keep moving in straight line. Under these constraints and conditions, controller uses the objective stated in (4-16) to determine the optimal reference trajectory at T2. There may be no solutions, one solution or multiple solutions. If no solutions can be determined, $\mathrm{a}_{13}=0 \mathrm{~m} / \mathrm{s}^{2}$ and $\mathrm{dT} 3$ is determined by using $\mathrm{y}_{1}(\mathrm{~T} 3)=1.5^{*}$ LaneWidth. In this case, $\mathrm{w} 1$ is $\theta_{1}$ divided by dT3. Car 1's controller updates the reference trajectory once at T2 and then continuously applies the control action, w1 and a13, from T2 to T3 in steps of dtM1 second, where $\mathrm{T} 3$ is the time instance when $\mathrm{y} 1 \mathrm{~m}(\mathrm{~T} 3)=1.5 *$ LaneWidth. 
At T3, the controller updates the reference trajectory. Constraints (4-3), (4-4), (4-7), (4-8) to (4-9) and (4-12) to (4-15) are used to determine the feasible reference trajectory solutions. The reference trajectory at T3 refers to the time spent by the car 1 in remaining phases of the trajectory - dT4, dT5, dT6, and the acceleration of car 1 in phase 4 (a14) of the passing maneuver. It is assumed car 1 does not accelerate in phases 5 and 6. Angular orientation of car 1 at T5 $\left(\theta_{2}\right)$ is fixed to -30 degrees. For predicting trajectories of cars 2 and 3, car 1 uses measured position, velocity and acceleration of both cars at T3 to predict their future trajectories with the assumption that both cars continue with the same acceleration till T4, after which acceleration of both cars, a2 \& a3, is $0 \mathrm{~m} / \mathrm{s}^{2}$ and that they keep moving in straight line. Under these constraints and conditions, controller uses the objective stated in (4-16) to determine the optimal reference trajectory at T2. There may be no solutions, one solution or multiple solutions. If no solutions can be determined, $\mathrm{a}_{14}=\mathrm{a}_{1} \max$ if $\mathrm{v}_{1}$ is less than $\mathrm{V}_{\max }$. Car 1's controller continuously updates the reference trajectory and applies the control action, a14, from T3 to T4 in steps of dtM1 second.

At T4, the controller updates the reference trajectory. Constraints (4-3), (4-4), (4-7), (4-8), (4-9) and (4-14) to (4-15) are used to determine the feasible reference trajectory solutions. The reference trajectory at T4 refers to the time spent by the car 1 in remaining phases of the trajectory - dT5, dT6, angular orientation of car 1 at $\mathrm{T} 5\left(\theta_{2}\right)$ and the acceleration of car 1 in phase 5 (a15) of the passing maneuver. It is assumed car 1 does not accelerate in phase 6 . For predicting trajectories of cars 2 and 3, car 1 uses measured position and velocity of both cars at T4 to predict their future trajectories with the assumption that $\mathrm{a}_{2} \& \mathrm{a}_{3}$ is $0 \mathrm{~m} / \mathrm{s}^{2}$ and that they keep moving in straight line. 
Under these constraints and conditions, controller uses the objective stated in (4-16) to determine the optimal reference trajectory at $\mathrm{T} 4$. There may be no solutions, one solution or multiple solutions. If no solutions can be determined, $\mathrm{a}_{15}=0 \mathrm{~m} / \mathrm{s}^{2}, \theta_{2}=30$ degrees and dT5 is determined by using $\mathrm{y} 1(\mathrm{~T} 5)=$ LaneWidth. In this case, w1 is $\theta_{2}$ divided by dT5. Car 1's controller updates the reference trajectory once at T4 and then continuously applies the control action, w1 and a15, from T4 to T5 in steps of dtM1 second.

At T5, the controller updates the reference trajectory. Constraints (4-4), (4-8) to (4-9) and (4-15) are used to determine the feasible reference trajectory solutions. The reference trajectory at $\mathrm{T} 5$ refers to the time spent by the car 1 in remaining phase of the trajectory - dT6 and the acceleration of car 1 in phase 6 (a16) of the passing maneuver. For predicting trajectories of cars 2 and 3, car 1 uses measured position and velocity of both cars at T5 to predict their future trajectories with the assumption that $\mathrm{a}_{2} \& \mathrm{a}_{3}$ is $0 \mathrm{~m} / \mathrm{s}^{2}$ and that they keep moving in straight line. Under these constraints and conditions, controller uses the objective stated in (4-17) to determine the optimal reference trajectory at T5. The objective stated in (4-16) cannot be used to find the optimal reference trajectory as car 1 does not interact with car 3 after T5. So, only objective is to maximize distance between cars 1 and 2 at T6, as stated below.

$\max .\left[\mathrm{x}_{1}\left(\mathrm{~T}_{6}\right)-\mathrm{x}_{2}\left(\mathrm{~T}_{6}\right)\right]$

There may be no solutions, one solution or multiple solutions. If no solutions can be determined, $\mathrm{a}_{16}=0 \mathrm{~m} / \mathrm{s}^{2}$ and $\mathrm{dT} 6$ is determined by using $\mathrm{y} 1(\mathrm{~T} 6)=$ $0.5 *$ LaneWidth. In this case, w1 is $\theta_{2}$ divided by dT6. Car 1's controller updates the 
reference trajectory once at T5 and then continuously applies the control action, $\mathrm{w}_{1}$ and a16, from T5 to T6 in steps of dtM1 second, where T6 is the time instance when $\mathrm{y}_{1 \mathrm{~m}}(\mathrm{~T} 6)$ $=0.5 *$ LaneWidth. At T6, car 1 has safely completed the passing maneuver.

\subsection{Actuator}

The actuator functionally represents the actuator of the car 1 with the assumption that the execution of control signal is instantaneous. The actuator updates the dynamics of car 1 based on the control action received from the controller at periodic intervals of dtM1 second. The control action consists of desired acceleration and angular velocity of car 1 . The actuator uses the current dynamics of the car 1 , control action and equations (4-18) to (4-23) to update the dynamics of the car 1.

$$
\begin{aligned}
& \text { theta }(\mathrm{t}+1)=\operatorname{theta}(\mathrm{t})+\mathrm{w}_{1} * \text { dtM1 } \\
& \mathrm{v}_{1}(\mathrm{t}+1)=\mathrm{v}_{1}(\mathrm{t})+\mathrm{a}_{1} * \text { dtM1 } \\
& \mathrm{v}_{1 \mathrm{x}}(\mathrm{t}+1)=\mathrm{v}_{1}(\mathrm{t}+1) * \cos (\operatorname{theta}(\mathrm{t}+1)) \\
& \mathrm{v}_{1 \mathrm{y}}(\mathrm{t}+1)=\mathrm{v}_{1}(\mathrm{t}+1) * \sin (\operatorname{theta}(\mathrm{t}+1)) \\
& \mathrm{x}_{1}(\mathrm{t}+1)=\mathrm{x}_{1}(\mathrm{t})+\mathrm{v}_{1}(\mathrm{t}) * \frac{\sin (\operatorname{theta}(\mathrm{t}+1))}{\mathrm{w}_{1}}-\mathrm{v}_{1}(\mathrm{t}) * \frac{\sin (\operatorname{theta}(\mathrm{t}))}{\mathrm{w}_{1}}-\left(\mathrm{a}_{1} *\right. \\
& \frac{\cos (\operatorname{theta}(\mathrm{t}))}{\mathrm{w}_{1}^{2}}+\mathrm{a}_{1} * \frac{\mathrm{w}_{1} * \operatorname{dtM} 1 * \sin (\operatorname{theta}(\mathrm{t}+1))+\cos (\operatorname{theta}(\mathrm{t}+1))}{\mathrm{w}_{1}^{2}} \\
& \mathrm{y}_{1}(\mathrm{t}+1)=\mathrm{y}_{1}(\mathrm{t})-\mathrm{v}_{1}(\mathrm{t}) * \frac{\cos (\operatorname{theta}(\mathrm{t}+1))}{\mathrm{w}_{1}}+\mathrm{v}_{1}(\mathrm{t}) * \frac{\cos (\operatorname{theta}(\mathrm{t}))}{\mathrm{w}_{1}}-\left(\mathrm{a}_{1} *\right. \\
& \frac{\sin (\operatorname{theta}(\mathrm{t}))}{\mathrm{w}_{1}^{2}}+\mathrm{a}_{1} * \frac{-\mathrm{w}_{1} * \operatorname{dtm} 1 * \cos (\operatorname{theta}(\mathrm{t}+1))+\sin (\operatorname{theta}(\mathrm{t}+1))}{\mathrm{w}_{1}^{2}}
\end{aligned}
$$




\section{Chapter 6: Car 2}

This chapter describes the system-level architecture of the car 2.

\subsection{Car 2 Concept}

Car 2 is the human-driven vehicle that car 1 is trying to pass. As outlined in section 4.2 of the thesis, functionality of car 2 is to collaborate with car 1 to support the passing maneuver. Car 2 does not interact with car 1 till T3. At T3, car 2 starts collaborating with car 1 to support the passing maneuver. This collaborative nature of car 2 is indicated by the Boolean variable 'Collaborative2' in the 'Car2' block in Figure 17. If it is 'true', it indicates a cooperative driver, while 'false' indicates a neutral driver. For supporting the passing maneuver, car 2 needs measurements of parameters of cars 1 and 2, and road to determine the control action and then uses the actuator to actuate control action. Thus, car 2 has 3 functional components - 'Measurement System 2', 'Model Predictive Controller 2' and 'Actuator 2', as shown in Figure 17.

Measurement system is characterized by standard deviation of error in measurement of relative dynamics of other cars and dynamics of car 2. It is different for measurements of other cars and measurements about car 2. The reaction time of driver, denoted by 'dtM2', is also characteristics of measurement system. Model Predictive controller, referred to as controller, of car 2 is characterized by safety distance used to plan the trajectory for car 2, assumed orientation of car 1 at T5, and control action, i.e., acceleration of car 2. The actuator is characterized by the current dynamics of the car 2 and dynamics of car 2 at previous time instant. 
The flow of information amongst different functional components of car 2 is shown the internal block diagram of car 2 in Figure 19. The measurement system collects all the information from the environment and sends it to the controller via 'MeasuredParameters2' interface. The controller uses that information to generate the control signal and sends it to the actuator via 'ControlAction2' interface. Measurement system measures car 2's parameters. This flow of information occurs via 'Car2Parameters' interface. The data types and values carried in these interfaces is defined in Figure 18 along with the units of each data type.

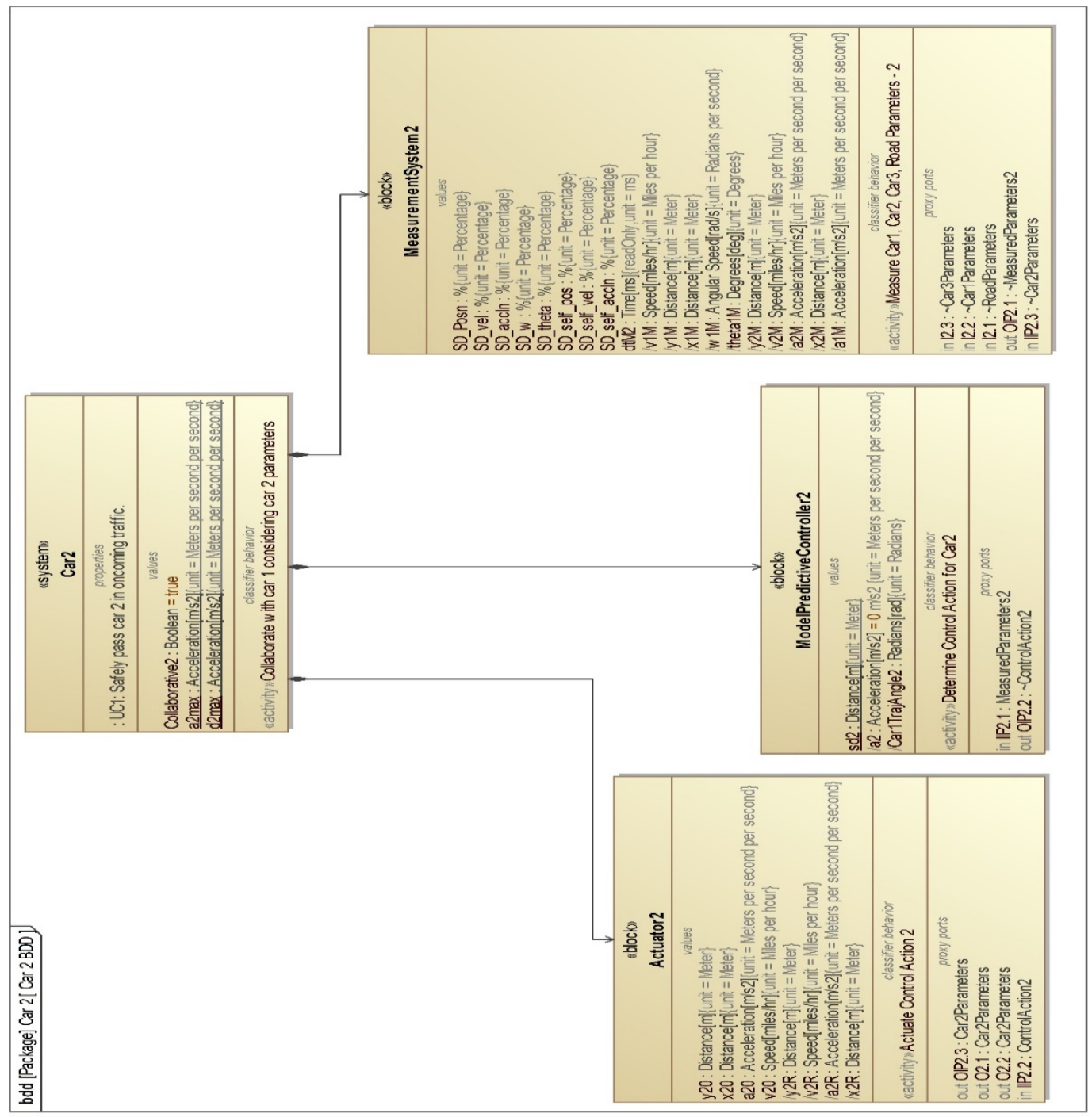

Figure 17: Car 2 Block Definition Diagram 


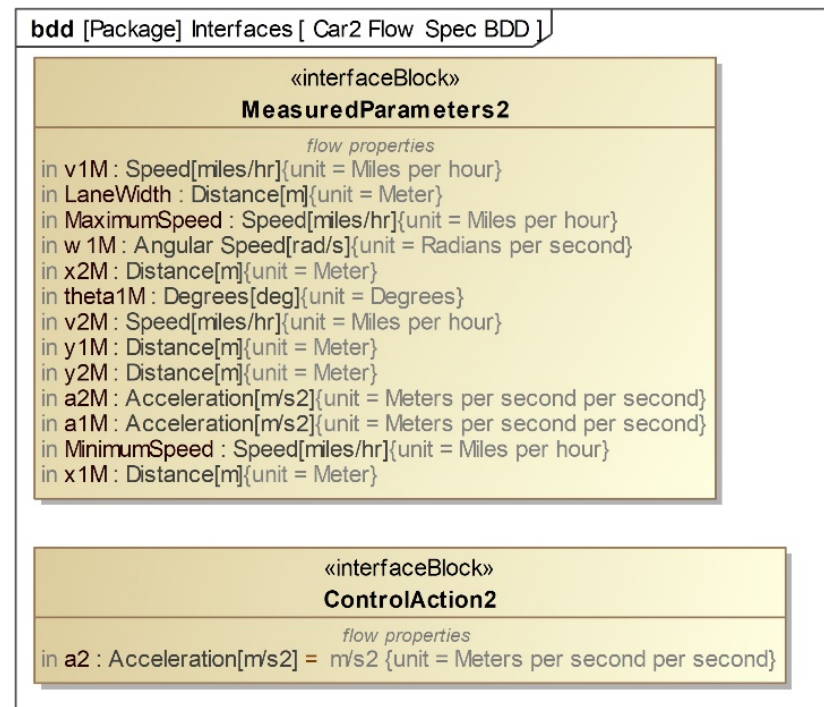

Figure 18: Car 2 Interface Block Definition Diagram

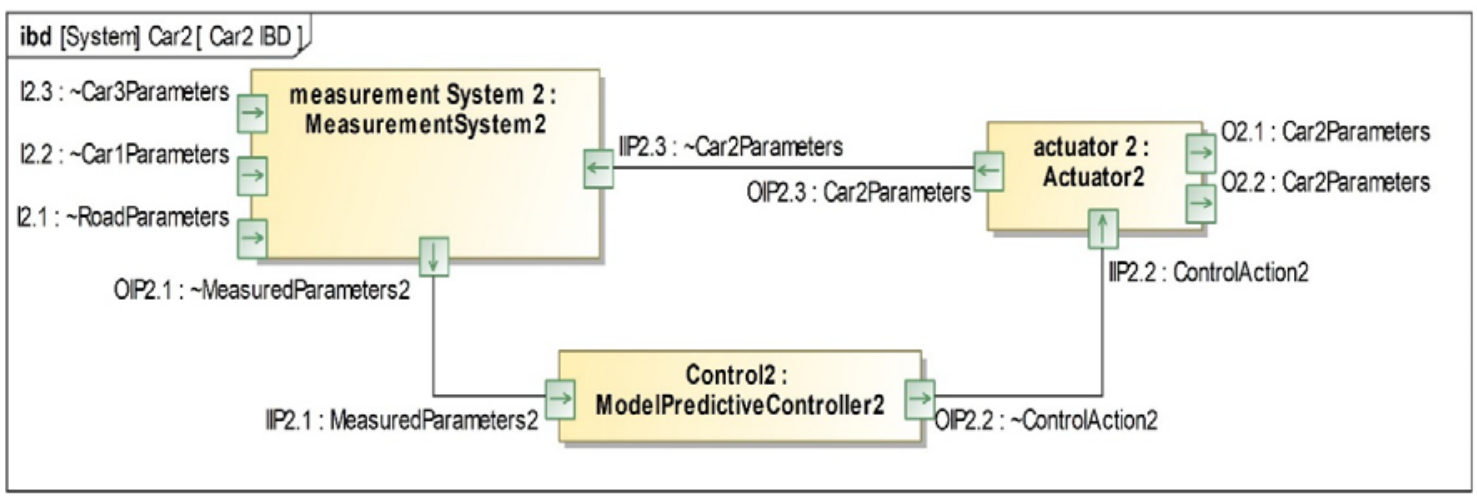

Figure 19: Car 2 Internal Block Diagram

The three functional components of car 2 perform different behaviors perceive, process information and react to it. The behaviors allocated to each are shown in Figure 20. This activity diagram is the decomposition of 'Collaborate with car 1 considering car 2 parameters' behavior allocated to car 2 . The activity parameters for the 'Collaborate with car 1 considering car 2 parameters' behavior, are shown on the diagram frame. Measurement system is allocated behavior - 'Measure car 1, car 2, car 3, road parameters - 2'. Controller executes the behavior - 'Determine control action for car 2'. This behavior outputs the control action. Actuator actuates the control action. The execution of each behavior is determined by the data flow. As soon as data is 
available at all the input ports of a behavior, behavior is executed. This means that multiplicity of all the input ports is 1 . These ports correspond to the ports on the internal block diagram of car 2 in Figure 19. All behaviors are executed instantaneously. After the execution of the behavior, the outputs of the behavior are available at the input ports downstream. Thus, the behaviors are executed sequentially, starting with when data is available to the 'Collaborate with car 1 considering car 2 parameters' behavior, i.e., activity input parameters on the diagram frame.

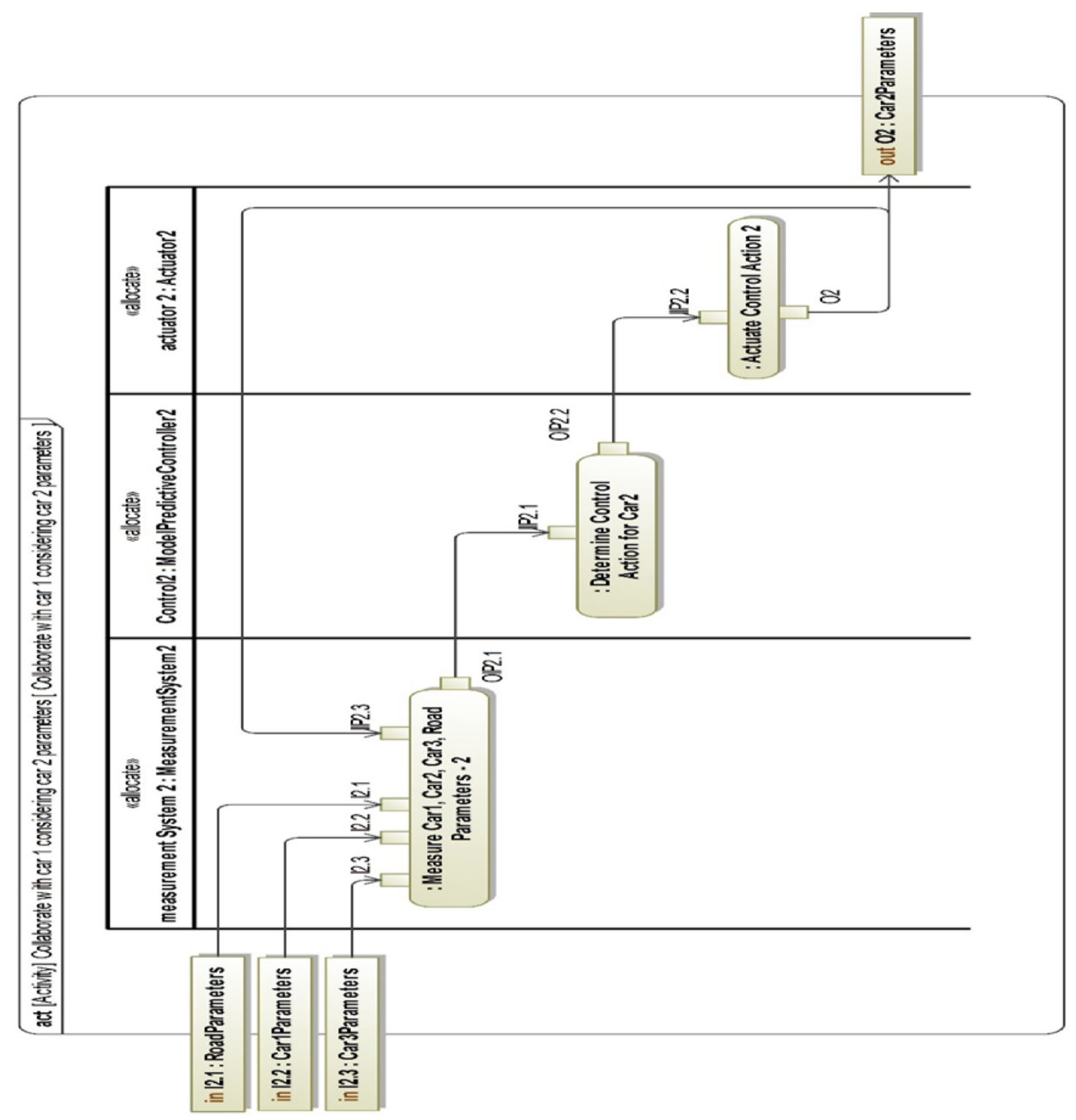

Figure 20: Car 2 Activity Diagram 


\subsection{Measurement System}

The measurement system of car 2 represents perception of the human driver. It takes measurements of all 3 cars and the road. These measurements are susceptible to measurement noise. In the model, this uncertainty in measurements has been modelled as white gaussian noise with zero mean and some standard deviation. This standard deviation of error in relative dynamics measurement is expressed as percentage of the relative dynamics of other cars and percentage of the absolute dynamics for car 2 , denoted by '_self' in the 'Measurement System 2' block in Figure 17. It is assumed to be $0 \%$ for measurements made by car 2 about dynamics of car 2 .

The measurement system measures car 2's position with reference as car 1's position at $\mathrm{T} 0$, absolute velocity and absolute acceleration. It measures relative position, relative velocity, relative acceleration, absolute angular orientation w.r.t. xaxis and absolute angular velocity of car 1 as well as dynamics of car 3 which are then used to calculate their absolute values. But only measurements of car 1 are sent to controller as only those are used to determine control action for car 2. It is assumed that measurement information is taken after every 'dtM2' second with no lag in information transfer at interfaces associated with the measurement system shown in Figure 19.

\subsection{Controller}

The function of controller is to determine control action for actuator of car 2 by calculating reference trajectory for car 2 based on measurement data from measurement system of car 2. The controller updates the reference trajectory and the control action every dtM2 seconds. It has 2 states - one state associated with time from T0 to T3 and 
T3 to T6 if it is neutral driver, referred to as state 1 , and other state associated with time from T3 to T6 if it is a cooperative driver, referred to as state 2. In state 1, control action or acceleration is $0 \mathrm{~m} / \mathrm{s}^{2}$. As per the assumptions stated in section 2.2, acceleration of car 2 is $0 \mathrm{~m} / \mathrm{s}^{2}$ from T0 to T3. From T0 to T6, if it a neutral driver, the acceleration is again $0 \mathrm{~m} / \mathrm{s}^{2}$. This control logic of the controller for different parts of the passing maneuver is shown in the state machine diagram in Figure 21. In state 2, 'State2/Phase4', 'State2/Phase5' and 'State2/Phase6' are associated with estimation of car 1's trajectory.

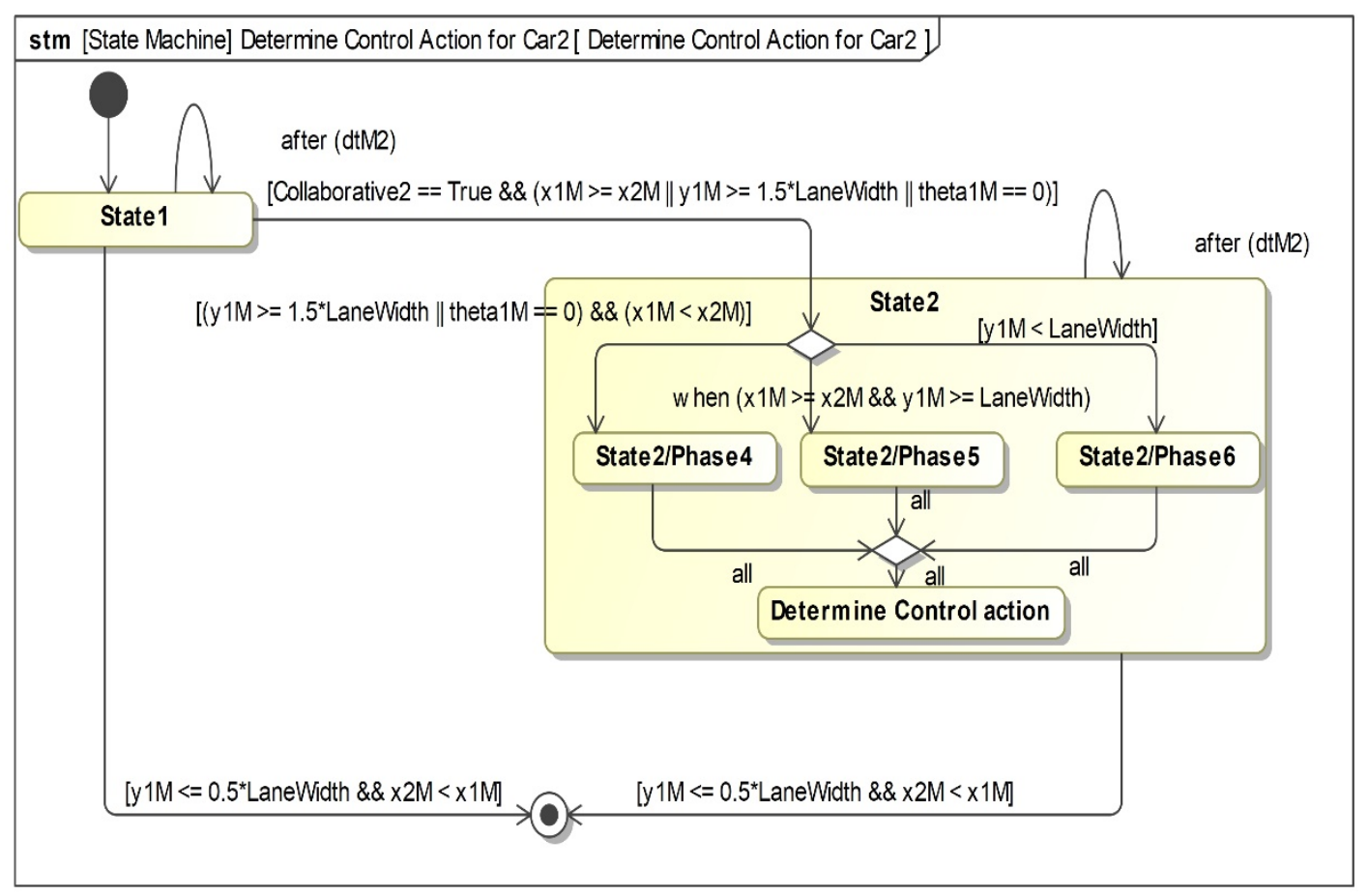

Figure 21: State Machine Diagram for Controller of Car 2

Each state of the trajectory has different set of constraints used to determine the control action for car 2. The guard conditions for entry to and exit from a state have been defined in the state machine diagram. The system goes to initial state at T0 and reaches final state when it determines from the measured data that car 1 has completed 
the passing maneuver. The state machine diagram for the controller identifies the behavior of the controller in different states as well as the paths to go from initial state to a final state. But this state control logic is susceptible to measurement noise among other factors. The control logic based on this state machine diagram is explained further in this section.

When car 1 has finished turning, i.e. at T3, controller determines that car 1 is trying to execute the passing maneuver. In that case, if car 2 is going to cooperate with car 1 to support the passing maneuver, controller of car 2 enters state 2 at T3. In this state, objective of car 2 is to support the passing maneuver. To support the passing maneuver, car 2's reference trajectory must satisfy three types of constraints - car safety constraints w.r.t. car 1, car 2 specification constraints and road safety constraints. All of these are inequality constraints. Car safety constraints ensure that car 2 maintains a safe distance of ' $\mathrm{sd}_{2}$ ' w.r.t. car 1 when it is in the same lane as car 2 after time instance T3, as stated below.

$$
\begin{aligned}
& x_{1}\left(T_{5}\right)-x_{2}\left(T_{5}\right) \geq s d_{2} \\
& x_{1}\left(T_{6}\right)-x_{2}\left(T_{6}\right) \geq s d_{2} \\
& v_{1 x}\left(T_{5}\right) \geq v_{2}\left(T_{5}\right)
\end{aligned}
$$

Car 2 collaborates with car 1 after T3, so, there are no safety constraints on car 2 before T3. Satisfying constraints (5-1) and (5-2) ensures safety at T5 and T6 but does not ensure the safety in between the time interval T5 to T6. Thus, constraint (5-3) is required to ensure the safety of the car 2 w.r.t. car 1 throughout the time interval - [T5, T6]. Constraint (5-3) combined with no positive acceleration of car 2 after T3 and 
increase in velocity of car 1 along $\mathrm{x}$-axis from T5 to T6 as it turns, ensures that car 2 at the least maintains the clearance distance it had at T5 w.r.t. car 1 from T5 through T6. Further, car 1 has minimum velocity along $\mathrm{x}$-axis at $\mathrm{T} 5$ in the time interval - [T5, T6] which means the clearance distance of car 2 w.r.t. car 1 will increase after T5 and will be greater than $\mathrm{sd}_{2}$ at $\mathrm{T} 6$, if it was greater than $\mathrm{sd}_{2}$ at $\mathrm{T} 5$. This makes constraint (5-2) redundant. Road safety constraints ensure that car 2 dynamics are within the speed limits for the road. As such the constraints are stated below.

$v_{\min } \leq v_{2}(t) \leq v_{\max }, \quad$ where $t \in\left[T_{3}, T_{6}\right]$

At T5, velocity of car 1 along $x$-axis may be less than $\mathrm{Vmin}_{\text {min }}$ so minimum velocity constraint on car 2 is $0 \mathrm{~m} / \mathrm{s}$, i.e., it can be less than $\mathrm{V}_{\text {min. }}$. Car 2 specification constraints ensure that deacceleration of car 2 is within the specifications. As car 2 never accelerates from $\mathrm{T} 3$ to $\mathrm{T} 6$, there are no constraints on positive acceleration. The constraints are stated below.

$0 \geq \mathrm{a}_{2}(\mathrm{t}) \geq-\mathrm{d}_{2 \max }, \quad$ where $\mathrm{t} \in\left[\mathrm{T}_{3}, \mathrm{~T}_{6}\right]$

In state 2 from T3 to T5, constraints (5-1) and (5-3) to (5-5) are used to determine the feasible reference trajectory. The reference trajectory refers to deacceleration of car 2 (a2) from T3 to T6 to support the passing maneuver. Under these constraints and conditions, there may be no solutions, one solution or multiple solutions. If there are no solutions, $\mathrm{a}_{2}=-\mathrm{d}_{2 \max }$. If there is one solution or multiple solutions, solution with minimum magnitude of deacceleration is chosen. Thus, the objective function is as stated in (5-6). 
$\operatorname{minimize}\left(\mathrm{a}_{2}\right)^{2}$

The objective stated in (5-6) is used to determine the optimal reference trajectory. It reflects that the driver slows down only as much as required. Controller continuously updates the reference trajectory and applies the control action, a2, from T3 to T5 in steps of dtM2 second, where T5 is the time instance when $\mathrm{y} 1 \mathrm{~m}(\mathrm{~T} 5) \leq$ LaneWidth and x1m > x2m. In state 2 from T5 to T6, constraints (5-2) to (5-5) are used to determine the feasible reference trajectory. The reference trajectory refers to deacceleration of car 2 (a2) from T5 to T6 to support the passing maneuver. Under these constraints and conditions, there may be no solutions, one solution or multiple solutions. If there are no solutions, $\mathrm{a}_{2}=-\mathrm{d}_{2 \max }$. If there is one solution or multiple solutions, solution with minimum magnitude of deacceleration is chosen using the objective function (5-6). Controller continuously updates the reference trajectory and applies the control action, a2, from T5 to T6 in steps of dtM2 second.

From this discussion, it's clear that the controller needs the information on car 1's trajectory to support the passing maneuver. State 2 of the controller incorporates a predictor model of car 1's passing maneuver which takes in measurements of car 1 dynamics from measurement system of car 2 and uses that to predict car 1's trajectory every dtM2 seconds. The model uses the measurements of car 1 dynamics to determines which phase of the passing maneuver car 1 is in. If $\mathrm{y} 1 \mathrm{~m} \geq 1.5 *$ LaneWidth or $\theta_{1 \mathrm{~m}}=0$ and $\mathrm{x} 1 \mathrm{~m}<\mathrm{x} 2 \mathrm{~m}$, car 1 is in phase 4 of the passing maneuver. The predictor model uses the current position, velocity and acceleration of cars 1 and 2 to determine dT4 using the constraint (5-7) and road safety constraint that $\mathrm{v}_{1}(\mathrm{t}) \leq \mathrm{v}_{\max }$ for all $\mathrm{t} \in[\mathrm{T} 3$, 
T4]. Then, it calculates velocity of car 1 at T4. This velocity, constraint (5-8), constraint (5-9), assumptions that orientation of car 1 at T5 is -30 degrees and $\mathrm{a}_{1}$ is $0 \mathrm{~m} / \mathrm{s}^{2}$ after T4, are used to calculate dT5 and dT6. These are used to determine $\mathrm{x}_{1}(\mathrm{~T} 5), \mathrm{x}_{1}(\mathrm{~T} 6)$, $\mathrm{x}_{2}(\mathrm{~T} 5), \mathrm{x}_{2}(\mathrm{~T} 6), \mathrm{v}_{1 x}(\mathrm{~T} 5)$ and $\mathrm{v}_{2}(\mathrm{~T} 5)$ which are used to calculate the control action.

$$
\begin{aligned}
& \mathrm{x}_{1}\left(\mathrm{~T}_{4}\right)=\mathrm{x}_{2}\left(\mathrm{~T}_{4}\right) \\
& \mathrm{y}_{1}\left(\mathrm{~T}_{5}\right)=\mathrm{lw} \\
& \mathrm{y}_{1}\left(\mathrm{~T}_{6}\right)=0.5 * \mathrm{lw}
\end{aligned}
$$

If $\mathrm{y}_{1 \mathrm{~m}} \geq$ LaneWidth and $\mathrm{x}_{1 \mathrm{~m}} \geq \mathrm{x}_{2} \mathrm{~m}$, car 1 is in phase 5 of the passing maneuver. The predictor model uses the current position, velocity and acceleration of car 1 to determine dT5 using the constraint (5-8) and road safety constraint that $\mathrm{v} 1 \mathrm{~m}(\mathrm{t}) \leq \mathrm{V} \max$ for all $t \in[\mathrm{T} 4, \mathrm{~T} 5]$. Then, it calculates velocity and orientation of car 1 at T5. This velocity, orientation of car 1 at T5, constraint (5-9) and assumption that a1 is $0 \mathrm{~m} / \mathrm{s}^{2}$ after T5, are used to calculate dT6. These are used to determine $\mathrm{x}_{1}(\mathrm{~T} 5), \mathrm{x}_{1}(\mathrm{~T} 6), \mathrm{x}_{2}(\mathrm{~T} 5)$, $\mathrm{x}_{2}(\mathrm{~T} 6), \mathrm{v}_{1 x}(\mathrm{~T} 5)$ and $\mathrm{v}_{2}(\mathrm{~T} 5)$ which are used to calculate the control action.

If $\mathrm{y} 1 \mathrm{~m}<$ LaneWidth and $\mathrm{x} 1 \mathrm{~m} \geq \mathrm{x} 2 \mathrm{~m}$, car 1 is in phase 6 of the passing maneuver. The predictor model uses the current position, velocity and acceleration of car 1 to determine dT6 using the constraint (5-9) and road safety constraint that $\mathrm{v} 1 \mathrm{~m}(\mathrm{t}) \leq \mathrm{V} \max$ for all $\mathrm{t} \epsilon[\mathrm{T} 5, \mathrm{~T} 6]$. These are used to determine $\mathrm{x}_{1}(\mathrm{~T} 6)$ and $\mathrm{x}_{2}(\mathrm{~T} 6)$ which are used to calculate the control action.

\subsection{Actuator}

The actuator functionally represents the actuator of the car 2 with the assumption that the execution of control signal is instantaneous. The actuator updates 
the dynamics of car 2 based on the control action received from the controller at periodic intervals of dtM2 second. The control action consists of desired acceleration of car 2. The actuator uses the current dynamics of the car 2, control action and equations (5-10) to (5-11) to update the dynamics of the car 2.

$$
\begin{aligned}
& \mathrm{v}_{2}(\mathrm{t}+1)=\mathrm{v}_{2}(\mathrm{t})+\mathrm{a}_{2} * d \mathrm{dtM} 2 \\
& \mathrm{x}_{2}(\mathrm{t}+1)=\mathrm{x}_{2}(\mathrm{t})+\mathrm{v}_{2}(\mathrm{t}) * d \mathrm{~d} M 2+\mathrm{a}_{2} * \frac{d \mathrm{tM} 2^{2}}{2}
\end{aligned}
$$




\section{Chapter 7: Car 3}

This chapter describes the system-level architecture of the car 3.

\subsection{Car 3 Concept}

Car 3 is part of human-driven oncoming traffic that car 1 must avoid crashing with while trying to pass. As outlined in section 4.2 of the thesis, functionality of car 3 is to collaborate with car 1 to support the passing maneuver. Car 3 does not interact with car 1 till T3. At T3, car 3 starts collaborating with car 1 to support the passing maneuver. This collaborative nature of car 3 is indicated by the Boolean variable 'Collaborative3' in the 'Car3' block in Figure 22. If it is 'true', it indicates a cooperative driver, while 'false' indicates a neutral driver. For supporting the passing maneuver, car 3 needs measurements of parameters of all three cars and road to determine the control action and then uses the actuator to actuate control action. Thus, car 3 has 3 functional components - 'Measurement System 3', ‘Model Predictive Controller 3’ and 'Actuator 3', as shown in Figure 22.

Measurement system is characterized by standard deviation of error in measurement of dynamics of other cars and dynamics of car 3. Standard deviation of error in measurement of relative dynamics is different for measurements of other cars and measurements about car 3. The reaction time of driver, denoted by 'dtM3', is also characteristics of measurement system. Model Predictive controller, referred to as controller, of car 3 is characterized by safety distance used to plan the trajectory for car 3, assumed orientation of car 1 at T5, and control action, i.e., acceleration of car 3. The 
actuator is characterized by the current dynamics of the car 3 and dynamics of car 3 at previous time instant.

The flow of information amongst different functional components of car 3 is shown the internal block diagram of car 3 in Figure 24. The measurement system collects all the information from the environment and sends it to the controller via 'MeasuredParameters3' interface. The controller uses that information to generate the control signal and sends it to the actuator via 'ControlAction3' interface. Measurement system measures car 3's parameters. This flow of information occurs via 'Car3Parameters' interface. The data types and values carried in these interfaces is defined in Figure 23 along with the units of each data type.

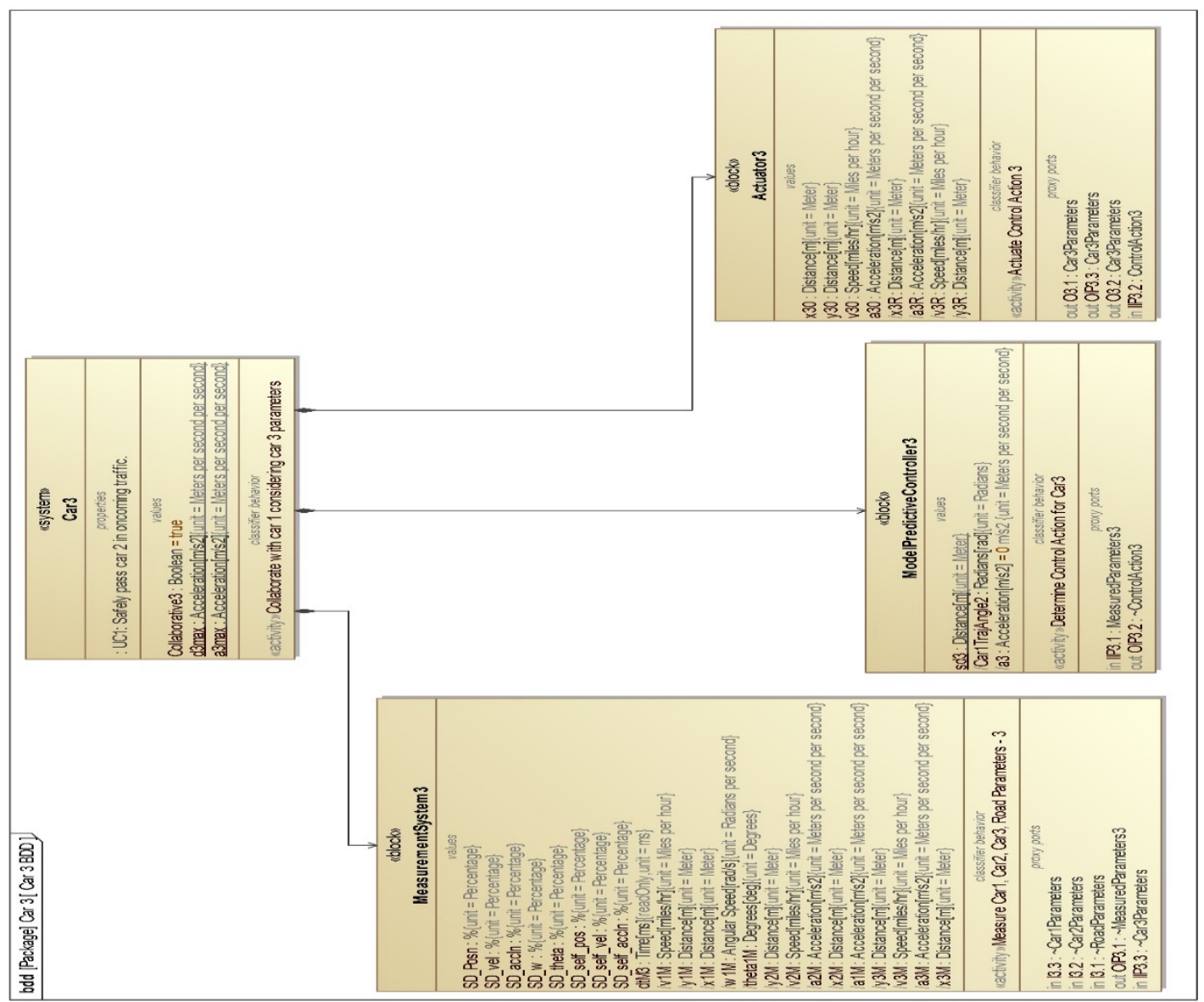

Figure 22: Car 3 Block Definition Diagram 


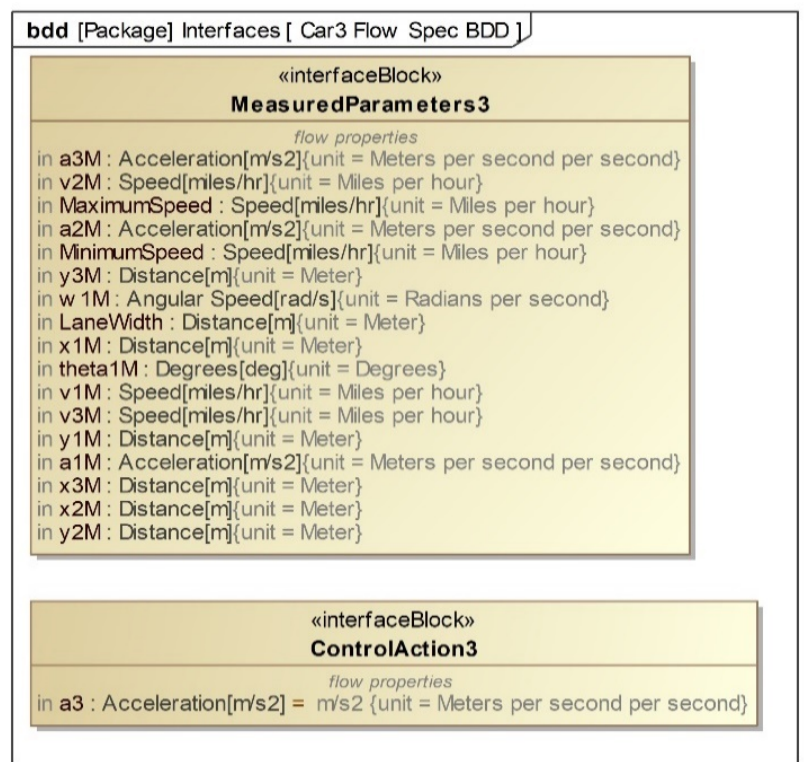

Figure 23: Car 3 Interface Block Definition Diagram

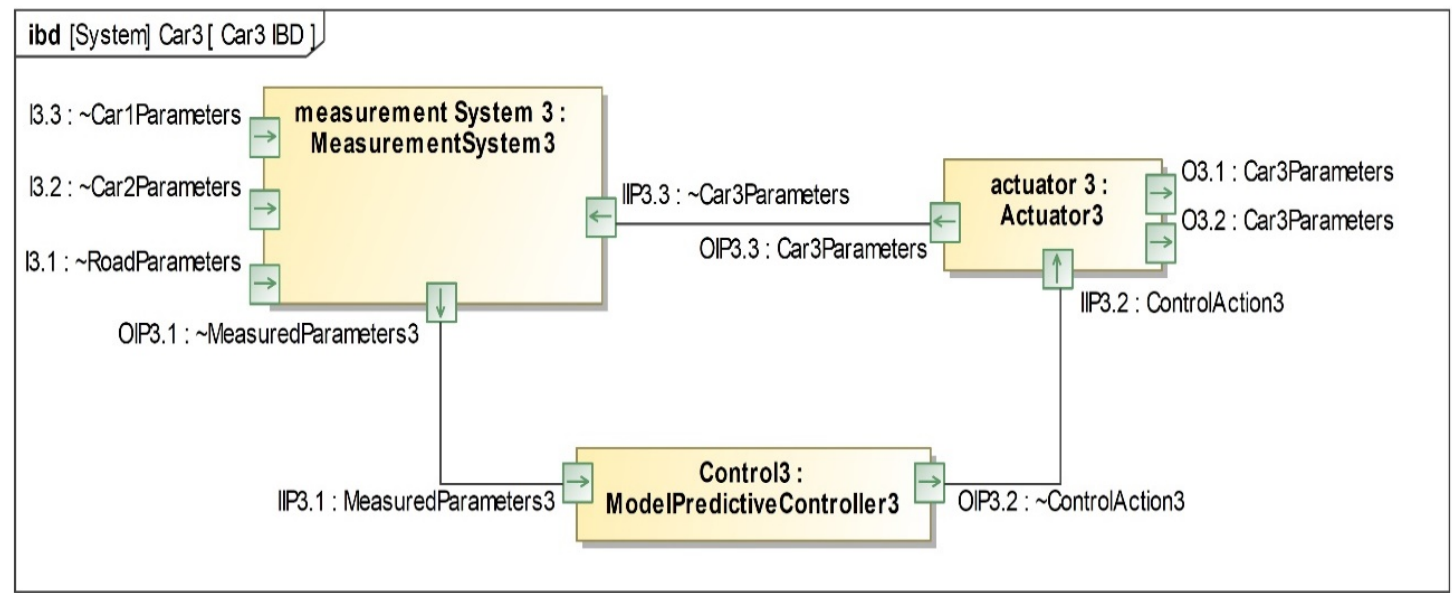

Figure 24: Car 3 Internal Block Diagram

The three functional components of car 3 perform different behaviors perceive, process information and react to it. The behaviors allocated to each are shown in Figure 25. This activity diagram is the decomposition of 'Collaborate with car 1 considering car 3 parameters’ behavior allocated to car 3. The activity parameters for the behavior, are shown on the diagram frame. Measurement system is allocated behavior - 'Measure car 1, car 2, car 3, road parameters - 3'. Controller executes the behavior - 'Determine control action for car 3'. This behavior outputs the control 
action. Actuator actuates the control action. The execution of each behavior is determined by the data flow. As soon as data is available at all the input ports of a behavior, behavior is executed, i.e., multiplicity of all the input ports is 1 . These ports correspond to the ports on the internal block diagram of car 3. It is assumed that all behaviors are executed instantaneously. After the execution of the behavior, the outputs of the behavior are available at the input ports downstream. Thus, the behaviors are executed sequentially, starting with when data is available to the 'Collaborate with car 1 considering car 3 parameters’ behavior.

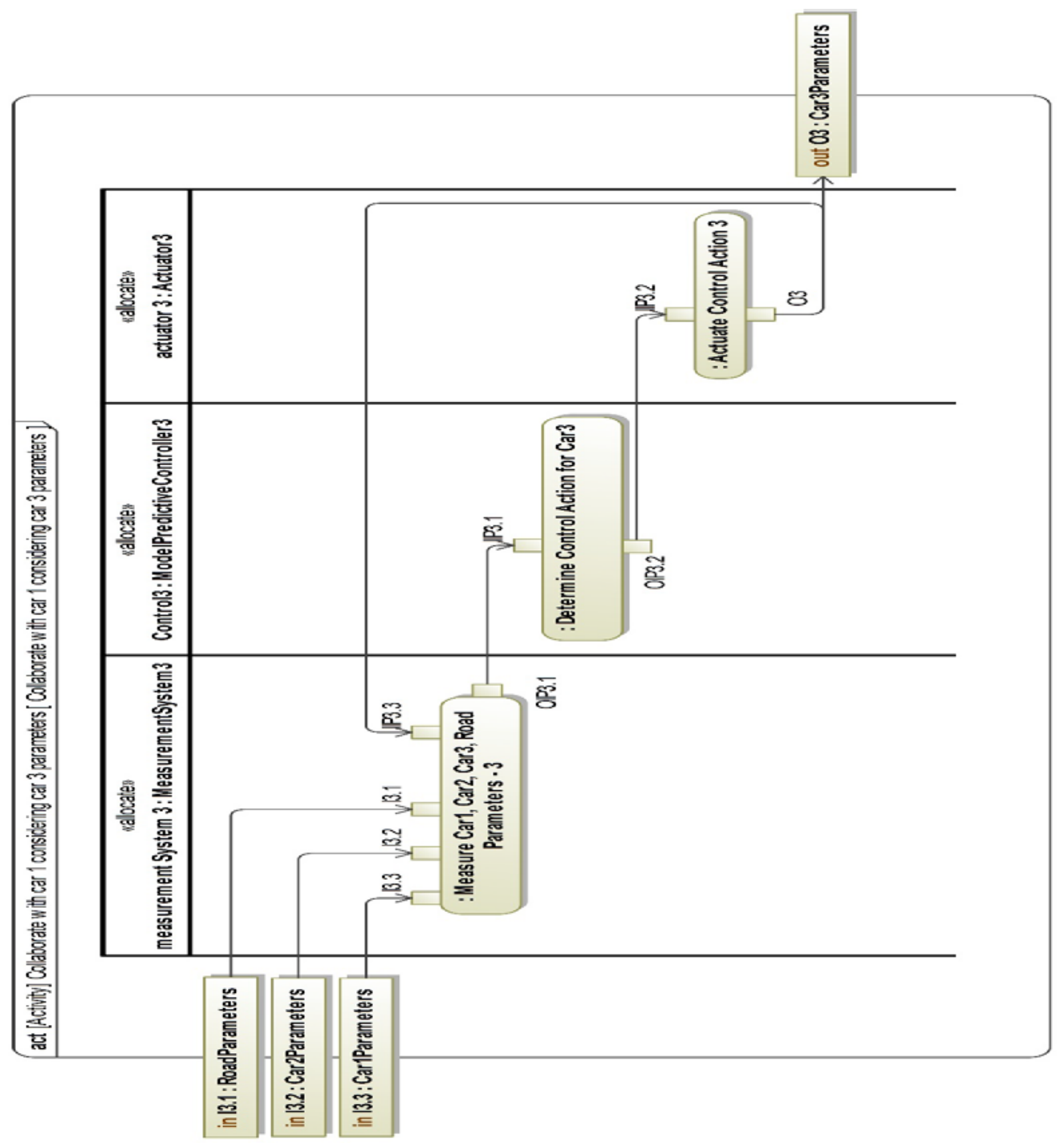

Figure 25: Car 3 Activity Diagram

60 


\subsection{Measurement System}

The measurement system of car 3 represents perception of the human driver. It takes measurements of all 3 cars and the road. These measurements are susceptible to measurement noise. In the model, this uncertainty in measurements has been modelled as white gaussian noise with zero mean and some standard deviation. This standard deviation is assumed to be $0 \%$ for measurements made by car 3 about dynamics of car 3. The measurement system measures car 3's position with reference as car 1's position at T0, absolute velocity and absolute acceleration. It measures relative position, relative velocity, relative acceleration, absolute angular orientation w.r.t. x-axis and absolute angular velocity of car 1 as well as dynamics of car 3 which are then used to calculate their absolute values. All these measurements are sent to the controller. It is assumed that measurement information is taken after every 'dtM3' second with no lag in information transfer at interfaces associated with the measurement system shown in Figure 24.

\subsection{Controller}

The function of controller is to determine control action for actuator of car 3 by calculating reference trajectory for car 3 based on measurement data from measurement system of car 3. The controller updates the reference trajectory and the control action every dtM3 seconds. It has 2 states - one state associated with time from T0 to T3 and T3 to T5 if it is neutral driver, referred to as state 1 , and other state associated with time from T3 to T5 if it is a cooperative driver, referred to as state 2. In state 1, control action or acceleration is $0 \mathrm{~m} / \mathrm{s}^{2}$. As per the assumptions stated in section 2.2, acceleration of 
car 3 is $0 \mathrm{~m} / \mathrm{s}^{2}$ from T0 to T3. From T0 to T5, if it a neutral driver, the acceleration is again $0 \mathrm{~m} / \mathrm{s}^{2}$. This control logic of the controller for different parts of the passing maneuver is shown in the state machine diagram in Figure 25. In state 2, 'State2/Phase4' and 'State2/Phase5' are associated with estimation of trajectories of cars 1 and 2.

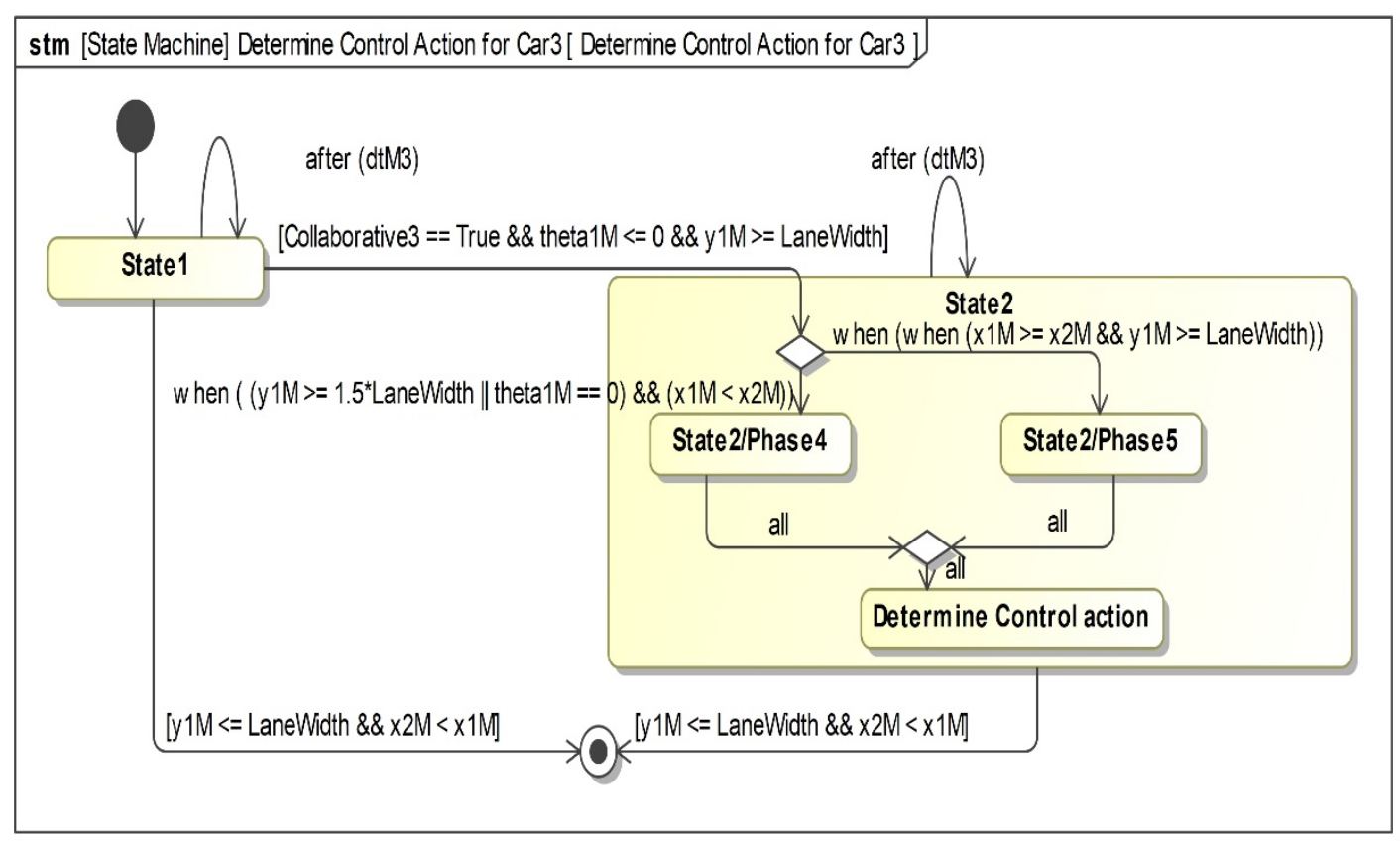

Figure 26: State Machine Diagram for Controller of Car 3

Each state of the trajectory has different set of constraints used to determine the control action for car 3. The guard conditions for entry to and exit from a state have been defined in the state machine diagram. The system goes to initial state at T0 and reaches final state when it determines from the measured data that car 1 has changed lanes after passing car 2, i.e., at T5. The state machine diagram for the controller identifies the behavior of the controller in different states as well as the paths to go from initial state to a final state. But this state control logic is susceptible to measurement noise among other factors. The control logic based on this state machine diagram is explained further in this section. 
When car 1 has finished turning, i.e. at T3, controller determines that car 1 is trying to execute the passing maneuver. In that case, if car 3 is going to cooperate with car 1 to support the passing maneuver, controller of car 3 enters state 2 at T3. In this state, objective of car 3 is to support the passing maneuver. To support the passing maneuver, car 3’s reference trajectory must satisfy three types of constraints - car safety constraints w.r.t. car 1, car 3 specification constraints and road safety constraints. All of these are inequality constraints. Car safety constraints ensure that car 3 maintains a safe distance of 'sd3' w.r.t. car 1 when it is in the same lane as car 3 after time instance T3, as stated below.

$\mathrm{x}_{3}\left(\mathrm{~T}_{5}\right)-\mathrm{x}_{1}\left(\mathrm{~T}_{5}\right) \geq \mathrm{sd}_{3}$

Car 3 collaborates with car 1 after T3, so, there are no safety constraints on car 3 before T3. Road safety constraints ensure that car 3 dynamics are within the speed limits for the road, as stated below.

$-\mathrm{v}_{\max } \leq \mathrm{v}_{3}(\mathrm{t}) \leq-\mathrm{v}_{\min }, \quad$ where $\mathrm{t} \in\left[\mathrm{T}_{3}, \mathrm{~T}_{5}\right]$

Car 3 specification constraints ensure that deacceleration of car 3 is within the specifications. As car 3 never accelerates from T3 to T5, there are no constraints on acceleration, as stated below.

$0 \leq \mathrm{a}_{3}(\mathrm{t}) \leq \mathrm{d}_{3 \max }, \quad$ where $\mathrm{t} \in\left[\mathrm{T}_{3}, \mathrm{~T}_{5}\right]$

In state 2, constraints (6-1) to (6-3) are used to determine the feasible reference trajectory. The reference trajectory refers to deacceleration of car 3 (a3) from T3 to T5 to support the passing maneuver. Under these constraints and conditions, there may be 
no solutions, one solution or multiple solutions. If there are no solutions, $\mathrm{a}_{3}=\mathrm{d}_{3 \max }$. If there is one solution or multiple solutions, solution with minimum magnitude of deacceleration is chosen. Thus, the objective function is as stated in (6-4).

$\operatorname{minimize}\left(\mathrm{a}_{3}\right)^{2}$

The objective stated in (6-4) is used to determine the optimal reference trajectory. It reflects that the driver slows down only as much as required. Controller continuously updates the reference trajectory and applies the control action, a3, from $\mathrm{T} 3$ to $\mathrm{T} 5$ in steps of dtM3 second, where $\mathrm{T} 5$ is the first time instance when $\mathrm{y} 1 \mathrm{~m}(\mathrm{~T} 5)=$ LaneWidth. From this discussion, it's clear that the controller needs the information on car 1's trajectory to support the passing maneuver. State 2 of the controller incorporates a predictor model of car 1's passing maneuver which takes in measurements of car 1 and car 2 dynamics from measurement system of car 3 and uses that to predict car 1's trajectory at every dtM3 seconds.

The model uses the measurements of car 1 and car 2 dynamics to determines which phase of the passing maneuver car 1 is in. If $y 1 \mathrm{~m} \geq 1.5^{*}$ LaneWidth or $\theta_{1 \mathrm{~m}}=0$ and $\mathrm{x}_{1 \mathrm{~m}}<\mathrm{x}_{2} \mathrm{~m}$, car 1 is in phase 4 of the passing maneuver. The predictor model uses the current position, velocity and acceleration of cars 1 and 2 to determine dT4 using the constraint (6-5) and road safety constraint that $\mathrm{v} 1(\mathrm{t}) \leq \mathrm{Vmax}$ for all $\mathrm{t} \epsilon[\mathrm{T} 3$, T4]. Then, it calculates velocity of car 1 at T4. This velocity, constraint (6-6), assumptions that orientation of car 1 at T5 is -30 degrees and $\mathrm{a}_{1}$ is $0 \mathrm{~m} / \mathrm{s}^{2}$ after $\mathrm{T} 4$, are used to calculate dT5. These are used to determine $\mathrm{x}_{1}(\mathrm{~T} 5)$ and $\mathrm{x}_{3}(\mathrm{~T} 5)$ which are used to calculate the control action. 


$$
\begin{aligned}
& \mathrm{x}_{1}\left(\mathrm{~T}_{4}\right)=\mathrm{x}_{2}\left(\mathrm{~T}_{4}\right) \\
& \mathrm{y}_{1}\left(\mathrm{~T}_{5}\right)=\mathrm{lw}
\end{aligned}
$$

If $\mathrm{y}_{1 \mathrm{~m}} \geq$ LaneWidth and $\mathrm{x} 1 \mathrm{~m} \geq \mathrm{x} 2 \mathrm{~m}$, car 1 is in phase 5 of the passing maneuver. The predictor model uses the current position, velocity and acceleration of car 1 to determine dT5 using the constraint (6-6) and road safety constraint that $\mathrm{v}_{1}(\mathrm{t}) \leq \mathrm{v}_{\max }$ for all $t \in[T 4, T 5]$. These are used to determine $\mathrm{x}_{1}(\mathrm{~T} 5)$ and $\mathrm{x}_{3}(\mathrm{~T} 5)$ which are used to calculate the control action.

\subsection{Actuator}

The actuator functionally represents the actuator of the car 3 with the assumption that the execution of control signal is instantaneous. The actuator updates the dynamics of car 3 based on the control action received from the controller at periodic intervals of dtM3 second. The control action consists of desired acceleration

of car 3. The actuator uses the current dynamics of the car 3, control action and equations (6-7) to (6-8) to update the dynamics of the car 3.

$$
\begin{aligned}
& \mathrm{v}_{3}(\mathrm{t}+1)=\mathrm{v}_{3}(\mathrm{t})+\mathrm{a}_{3} * d \mathrm{dtM} 3 \\
& \mathrm{x}_{3}(\mathrm{t}+1)=\mathrm{x}_{3}(\mathrm{t})+\mathrm{v}_{3}(\mathrm{t}) * \mathrm{dtM} 3+\mathrm{a}_{3} * \frac{\mathrm{dtM} 3^{2}}{2}
\end{aligned}
$$




\section{Chapter 8: Simulation}

This chapter describes the values assigned to different characteristics of the elements of the car passing problem as well as various iterations in the development of the executable model for car passing problem.

\subsection{Simulation Overview}

The mathematical model of the car passing problem described so far was built in MATLAB with the same structure as the structure and behavior as described in chapters 4 through 7 . For executing the model to do analysis, following values are assigned to different parameters.

- 'Collaborative2' and 'Collaborative3' are chosen randomly for each run of the simulation.

- All cars have same dimensions. So, safety distance between cars in same lane is considered same, i.e., sd $=5 \mathrm{~m}$ and safe distance used by the cars for trajectory planning is considered same, i.e., $s d_{i}=10 \mathrm{~m}$ for car ' $\mathrm{i}$ '.

- All cars have same dynamics, i.e., $\mathrm{a}_{1 \max }=\mathrm{a}_{2} \max =\mathrm{a}_{3 \max }=2.77 \mathrm{~m} / \mathrm{s}^{2}$ and $\mathrm{d}_{1 \max }=\mathrm{d}_{2 \max }$ $=\mathrm{d}_{3 \max }=10 \mathrm{~m} / \mathrm{s}^{2}$. It's based on assuming cars as 'Toyota Camry CE' [15].

- Assumed angular orientation of car 1 at $\mathrm{T} 2\left(\theta_{1}\right)$ is fixed at 30 degrees in phase 1 of the trajectory. Assumed angular orientation of car 1 at T5 $\left(\theta_{2}\right)$ is fixed at -30 degrees from phase 1 to 4 of the trajectory.

- Sampling time interval of the measurement system of car 1, to get measurements of the dynamics of other cars and itself, is 0.1 second. This is based on the sampling rate of LIDAR sensors [16]. 
- Cars 2 and 3 are human driven cars with a reaction time, dtM2 and dtM3, of 0.9 seconds [17].

- Maximum allowed speed on road, $\mathrm{V}_{\max }$, is $115 \mathrm{~km} / \mathrm{hr}$ and minimum allowed speed on road, Vmin, is $70 \mathrm{~km} / \mathrm{hr}$. This is based on the Maryland state highway speed limits [18].

- $\quad$ All lanes have same width, i.e., LaneWidth $=3 \mathrm{~m}$. This is based on the Maryland state highway designs [18].

- Velocities of car 1 and 2 are considered positive and velocity of car 3 is considered negative.

- Standard deviation of error in measurement of dynamics of a car by itself is $0 \%$.

- Standard deviation of error in measurement of position by cars 2 and 3 is assumed to be $8.6 \%$ [19].

- Standard deviation of error in measurement of velocity by cars 2 and 3 is assumed to be $10 \%$ [20].

- Standard deviation of error in measurement of acceleration by cars 2 and 3 is assumed to be $5 \%$.

- Standard deviation of error in measurement of angular orientation and angular velocity by cars 2 and 3 is $0 \%$.

All these values remain constant throughout the simulation. The design factors identified in section 3.3, standard deviation of error in position, velocity and acceleration measurement and clearance distance of car 1 w.r.t. cars 2 and 3 at T5, vary as discussed below. 
- Standard deviation of error in measurement of position by car 1 is $5 \%$ for monocular visual odometry [21] and 2.5\% for Universal Medium Range Radar sensor (nominal value) [22].

- Standard deviation of error in measurement of velocity by car 1 is $6 \%$ for visionbased constraint optical flow [23] and 2.91\% for Ultrasonic sensor system based on two-dimensional state method (nominal value) [24].

- Standard deviation of error in measurement of acceleration by car 1 is assumed to be either $5 \%$ or $2.5 \%$ (nominal value).

- Clearance distance of car 1 w.r.t. car 2 and 3 at T5 can take three values $-20 \mathrm{~m}$, $35 \mathrm{~m}$ (nominal value) and 50m. These are assumptions.

All these 72 cases are executed for 2 initial states- unsafe and safe - as discussed in section 3.3. The initial state of the system when it is safe to pass under the assumption that car 1 always has accurate measurements is described below, Table 1 .

Table 1: Initial State of the System when it is safe to pass

\begin{tabular}{|c|c|c|c|}
\hline $\mathrm{X} 1$ & $0 \mathrm{~m}$ & $\mathrm{y} 1$ & $1.85 \mathrm{~m}$ \\
\hline $\mathrm{X} 2$ & $200 \mathrm{~m}$ & y2 & $1.85 \mathrm{~m}$ \\
\hline $\mathrm{X}_{3}$ & $1300 \mathrm{~m}$ & уз & $5.55 \mathrm{~m}$ \\
\hline V1 & $100 \mathrm{~km} / \mathrm{hr}$ & $\mathrm{a}_{1}$ & $1 \mathrm{~m} / \mathrm{s}^{2}$ \\
\hline V2 & $76 \mathrm{~km} / \mathrm{hr}$ & $\mathrm{a}_{2}$ & $0 \mathrm{~m} / \mathrm{s}^{2}$ \\
\hline$V_{3}$ & $-75 \mathrm{~km} / \mathrm{hr}$ & a3 & $0 \mathrm{~m} / \mathrm{s}^{2}$ \\
\hline$\theta$ & $0 \mathrm{rad}$ & W1 & $0 \mathrm{rad} / \mathrm{s}$ \\
\hline
\end{tabular}


The initial state of the system when it is not safe to pass under the assumption that car 1 always has accurate measurements is described below, Table 2 .

Table 2: Initial State of the System when it is unsafe to pass

$\begin{array}{llll}\text { x1 } & 0 \mathrm{~m} & \text { y1 } & 1.85 \mathrm{~m} \\ \text { х2 } & 200 \mathrm{~m} & \text { y2 } & 1.85 \mathrm{~m} \\ \text { х3 } & 1100 \mathrm{~m} & \text { у3 } & 5.55 \mathrm{~m} \\ \text { v1 } & 100 \mathrm{~km} / \mathrm{hr} & \text { a1 } & 1 \mathrm{~m} / \mathrm{s}^{2} \\ \text { V2 } & 76 \mathrm{~km} / \mathrm{hr} & \text { a2 } & 0 \mathrm{~m} / \mathrm{s}^{2} \\ \text { v3 } & -75 \mathrm{~km} / \mathrm{hr} & \text { a3 } & 0 \mathrm{~m} / \mathrm{s}^{2} \\ \theta & 0 \mathrm{rad} & \text { w1 } & 0 \mathrm{rad} / \mathrm{s}\end{array}$

\subsection{Simulation Optimization}

The model described in the thesis is the final version of the model used for analysis. There were many iterations to get the model working right and fast. No formal verification and validation of the model was carried out, but model was informally checked and verified exhaustively by running it for different scenarios. As such there were many iterations of the model to reduce the errors in model execution and time for execution. The highlights of these iterations are documented below.

- In version 1 of the model, state 1 on the controller of car 1 had $\theta_{1}$ and $\theta_{2}$, in addition to dT1, dT2, dT3, dT4, dT5, dT6, a11 and $a_{14}$ as variables. This lead to long execution times for the nonlinear programming solver, 'fmincon', in MATLAB and most of the time the simulation crashed. To resolve this, the state variables for 
different states of the controller of car 1 were reduced and necessary assumptions were made. As such the controller of car 1 starts with an assumed orientation of the trajectory as well as assumed behavior for the future phases of the trajectory. This ensured that model executed every time, but execution time was still around 10 minutes for a safe initial state.

- Version 2 - To reduce the execution time further, bounds were defined for all the variables based on the results of one successful execution. $\theta_{1}$ and $\theta_{2}$ were bound between 15 degrees to 80 degrees and -15 degrees to -80 degrees, respectively. dT1 and dT4 have lower bound of 0 seconds and upper bound of 100 seconds. dT2, dT3, dT5 and dT6 have lower bound of 0 seconds and upper bound of 20 seconds. Rest of the variables were bounded by the design parameters of cars and their expected behavior - cars 2 and 3 are assumed to not accelerate. This brought down the average execution time to 4 minutes for a safe initial state.

- Version 3 - In version 2 of the model, controller of car 1 was updating the control action at every 0.1 seconds in phase 4 . On analysis of the control action, it was observed that control action remained almost constant throughout the phase 4 of the trajectory, as such control algorithm was modified to check the update the control action only once at the beginning of the phase. This reduced the average execution time to 1 minute for a safe initial state.

- Version 4 - Next major problem was that in many cases when it was safe to pass, controller determined the passing maneuver was not feasible due to measurement error. This was solved to some extent by putting in a condition that if the controller 
determines trajectory is infeasible for 2 consecutive points till T1, only then decision to not pass will be made.

- Version 5 - To reduce the execution time further, unnecessary execution of linear programming solver for cars 2 and 3 were reduced. Control actions for both cars are automatically assigned a value of ' 0 ' $\mathrm{m} / \mathrm{s}^{2}$ if it was determined the velocity of the car 1 was lower than or equal to minimum allowed velocity on road. This reduced the average execution time to 21 seconds for a safe initial state.

- Version 6 - Now the problem was that the controller moved to wrong states due to measurement noise, asynchronous information exchange among cars and measurement delay due to measurement system sampling rate. This was improved by updating the guard conditions for controller 1 from one shown in Figure 27 to one shown in Figure 16.

- Version 7 - Further robustness of the simulation was improved by ensuring that simulation doesn't stop unless two consecutive errors are encountered by any controller. In case of one error, control action from previous time instant is repeated.

This sums up the process of model and simulation optimization and troubleshooting for supporting the analysis. 


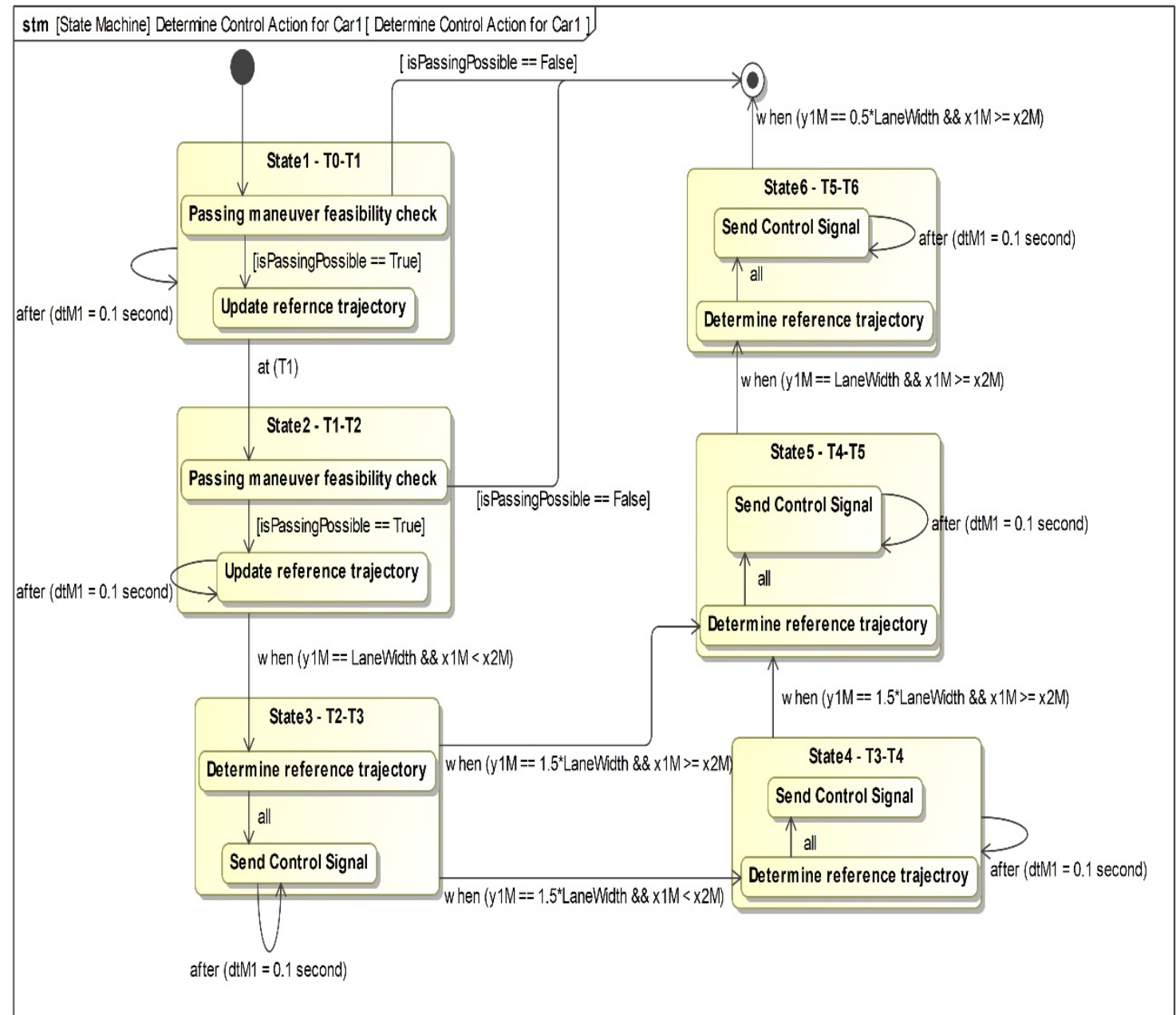

Figure 27: Earlier State Machine Diagram of Car 1 


\section{Chapter 9: Analysis, Results and Recommendations}

This chapter demonstrates that the executable model built by the author can be used for sensitivity analysis and trade-off analysis. It also presents various results collected regarding the problem form the executable model.

\subsection{Safe Trajectory analysis}

This section presents values of different parameters of the trajectory generated for the initial safe state. Standard deviation of error in position is $0 \%$, standard deviation of error in velocity is $0 \%$, standard deviation of error in acceleration is $0 \%$ and clearance distance of car 1 w.r.t. both cars 2 and 3 is $35 \mathrm{~m}$. The trajectory taken by three cars is shown in Figure 28. Car 1 spent 1.3 seconds in phase 1 of the trajectory. It took on average 18.1 seconds in completing the maneuver, i.e. dT2 + dT3 + dT4 + dT5 + dT6, with an average of 16.6 seconds spent in phase 4 of the trajectory. Controller determined the orientation of car 1 at $\mathrm{T} 1$ and $\mathrm{T} 5$ to be 20 degrees and -20 degrees respectively. Further it was observed that, car 1 accelerated to reach the maximum allowed velocity on road as early as possible.

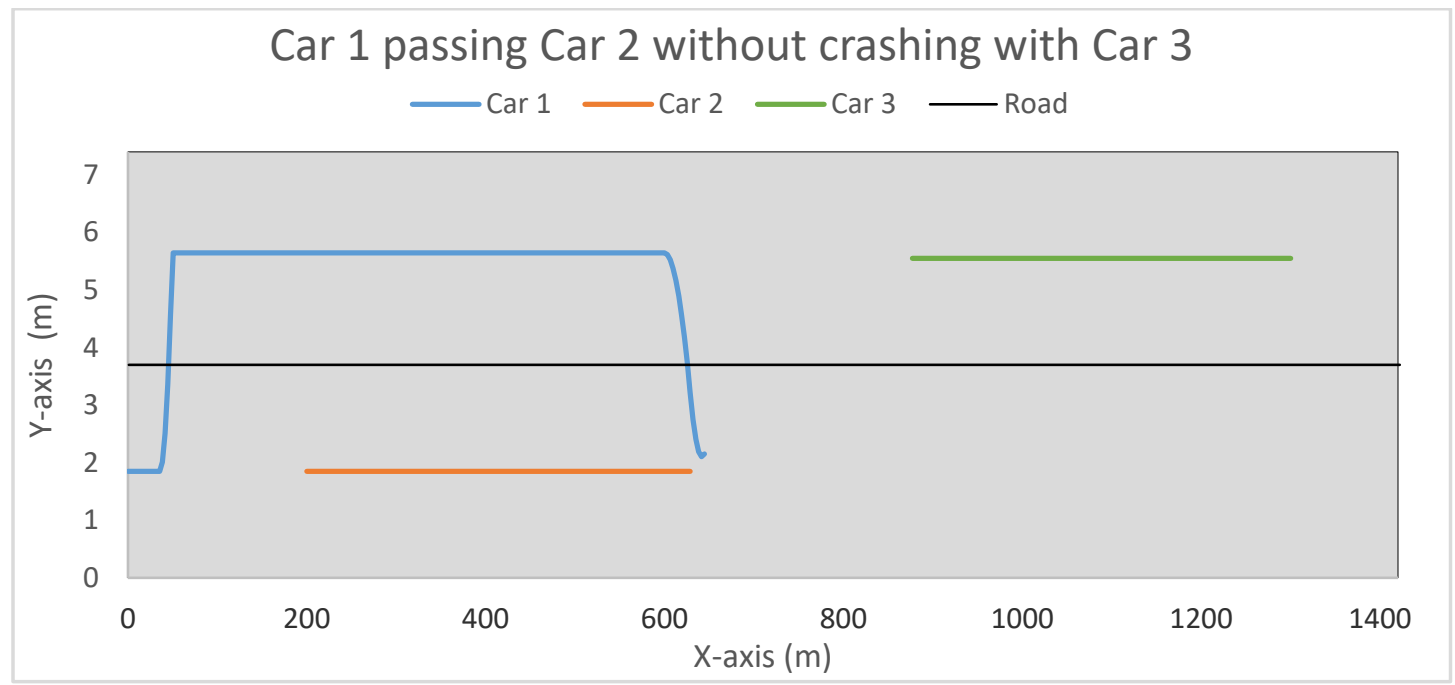

Figure 28: Car 1 successfully passing Car 2 without crashing with Car 3 


\subsection{Simulation Convergence Analysis}

Simulation data is to be collected for 144 cases including safe and unsafe initial state. It is critical to determine the minimum number of iterations for each case so that the output measurers, $\mathrm{P}(\mathrm{A}), \mathrm{P}(\mathrm{B})$ and $\mathrm{P}(\mathrm{C})$ converge. Initially, 500 iterations of the model for safe initial state and 400 iterations for unsafe initial states were executed separately and their standard error was recorded for nominal values of all parameters. For the nominal values of parameters, no case was found in which car1 crashed after deciding to pass (in 400 cases that were recorded) which complements the performance of the controller of car 1 . The corresponding graphs for $\mathrm{P}(\mathrm{A})$ and $\mathrm{P}(\mathrm{B})$ are shown in Figure 29 and Figure 30. Standard error for P(A), after 500, iterations is 0.0006 and 0.0007 after 400 iterations. Standard error for P(B), after 400 iterations, is 0.0014 .

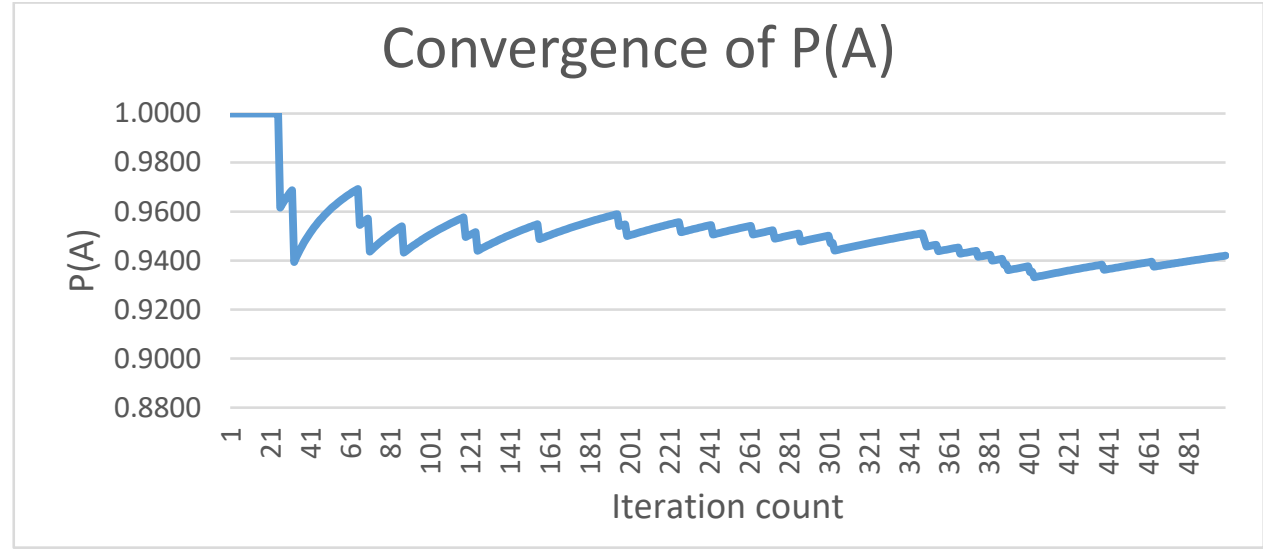

Figure 29: Convergence of $\mathrm{P}(\mathrm{A})$ with increase in iterations

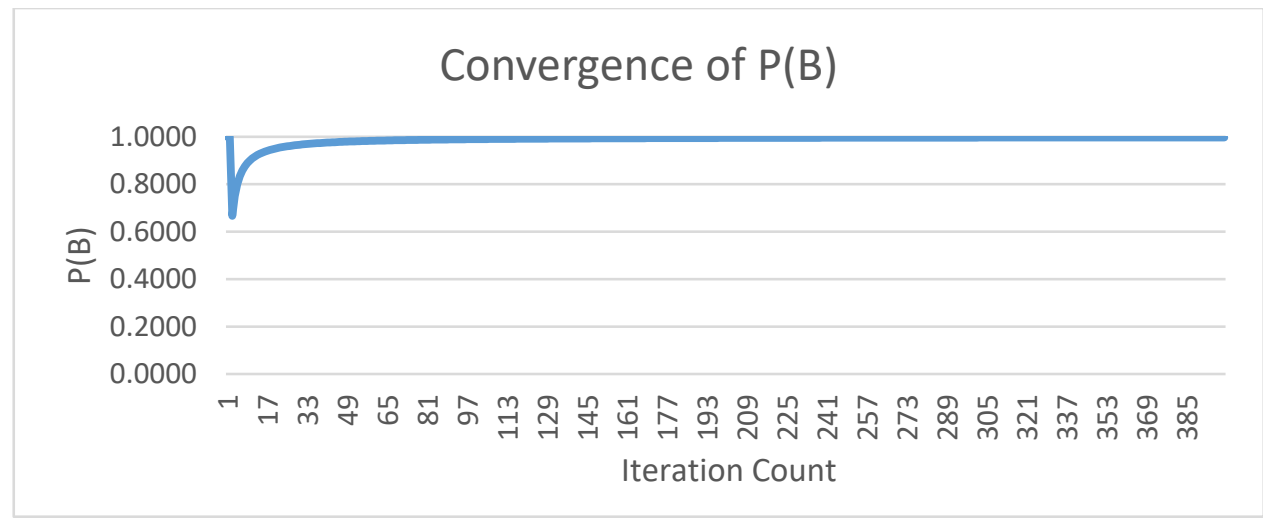

Figure 30: Convergence of $\mathrm{P}(\mathrm{B})$ with increase in iterations 


\subsection{Effect of Collaboration}

This section presents results on effects of collaboration amongst the drivers on safety of the passing maneuver given that car 1 decides to pass. To study the effects of collaboration, an initial state of the system was chosen for which passing is not possible when there is no collaboration by both cars 2 and 3. Further, the passing maneuver was executed till either there was a crash, or the passing maneuver was safely completed by car 1. This initial state is shown in Table 3. The trajectory corresponding to this initial state is shown in Figure 31 with car 1 crashing with car 3 in phase 4 of the trajectory. As car 2 collaborates with car 1 and slows down after T3, car 1 spends less time in phase 4 of the trajectory and can safely complete the passing maneuver as shown in Figure 32. When car 3 collaborates with car 1 and slows down after T3, car 1 has more clearance distance at T5 w.r.t. car 3 and can safely complete the passing maneuver, as shown in Figure 33. When both cars 2 and 3 collaborate, car 1 safely completes the passing maneuver by travelling less distance, as shown in Figure 34.

Table 3: Initial State of the System

\begin{tabular}{|l|l|l|l|}
\hline $\mathbf{x}_{\mathbf{1}}$ & $0 \mathrm{~m}$ & $\mathbf{y}_{1}$ & $1.85 \mathrm{~m}$ \\
\hline $\mathbf{x}_{\mathbf{2}}$ & $200 \mathrm{~m}$ & $\mathbf{y}_{2}$ & $1.85 \mathrm{~m}$ \\
\hline $\mathbf{x}_{\mathbf{3}}$ & $1000 \mathrm{~m}$ & $\mathbf{y}_{3}$ & $5.55 \mathrm{~m}$ \\
\hline $\mathbf{v}_{\mathbf{1}}$ & $100 \mathrm{~km} / \mathrm{hr}$ & $\mathbf{a}_{1}$ & $1 \mathrm{~m} / \mathrm{s}^{2}$ \\
\hline $\mathbf{v}_{\mathbf{2}}$ & $78 \mathrm{~km} / \mathrm{hr}$ & $\mathbf{a}_{2}$ & $0 \mathrm{~m} / \mathrm{s}^{2}$ \\
\hline $\mathbf{v}_{3}$ & $-80 \mathrm{~km} / \mathrm{hr}$ & $\mathbf{a}_{3}$ & $0 \mathrm{~m} / \mathrm{s}^{2}$ \\
\hline $\boldsymbol{\theta}$ & $0 \mathrm{rad}$ & $\mathbf{w}_{1}$ & $0 \mathrm{rad} / \mathrm{s}$ \\
\hline
\end{tabular}




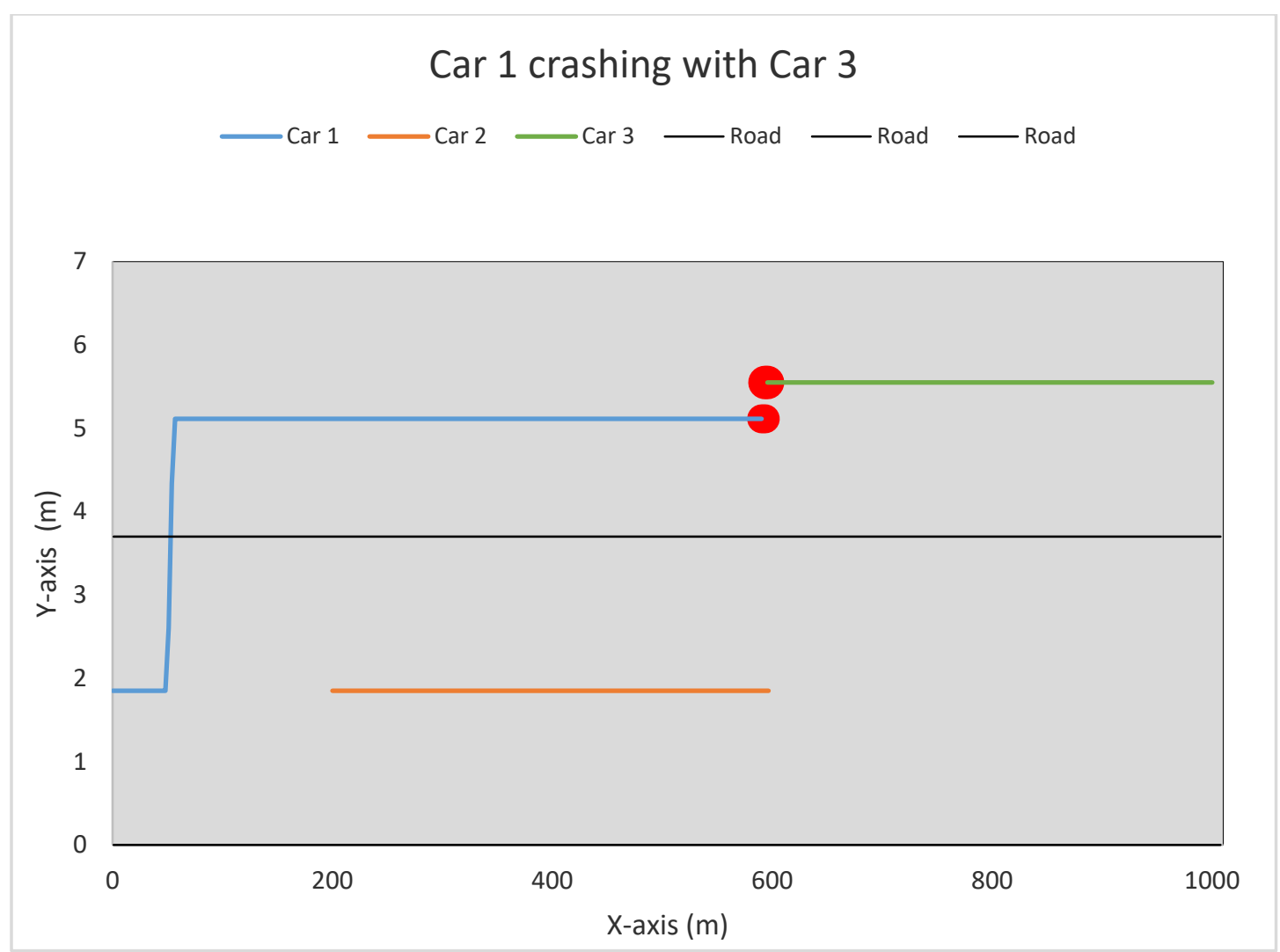

Figure 31: Car 1 crashing with Car 3

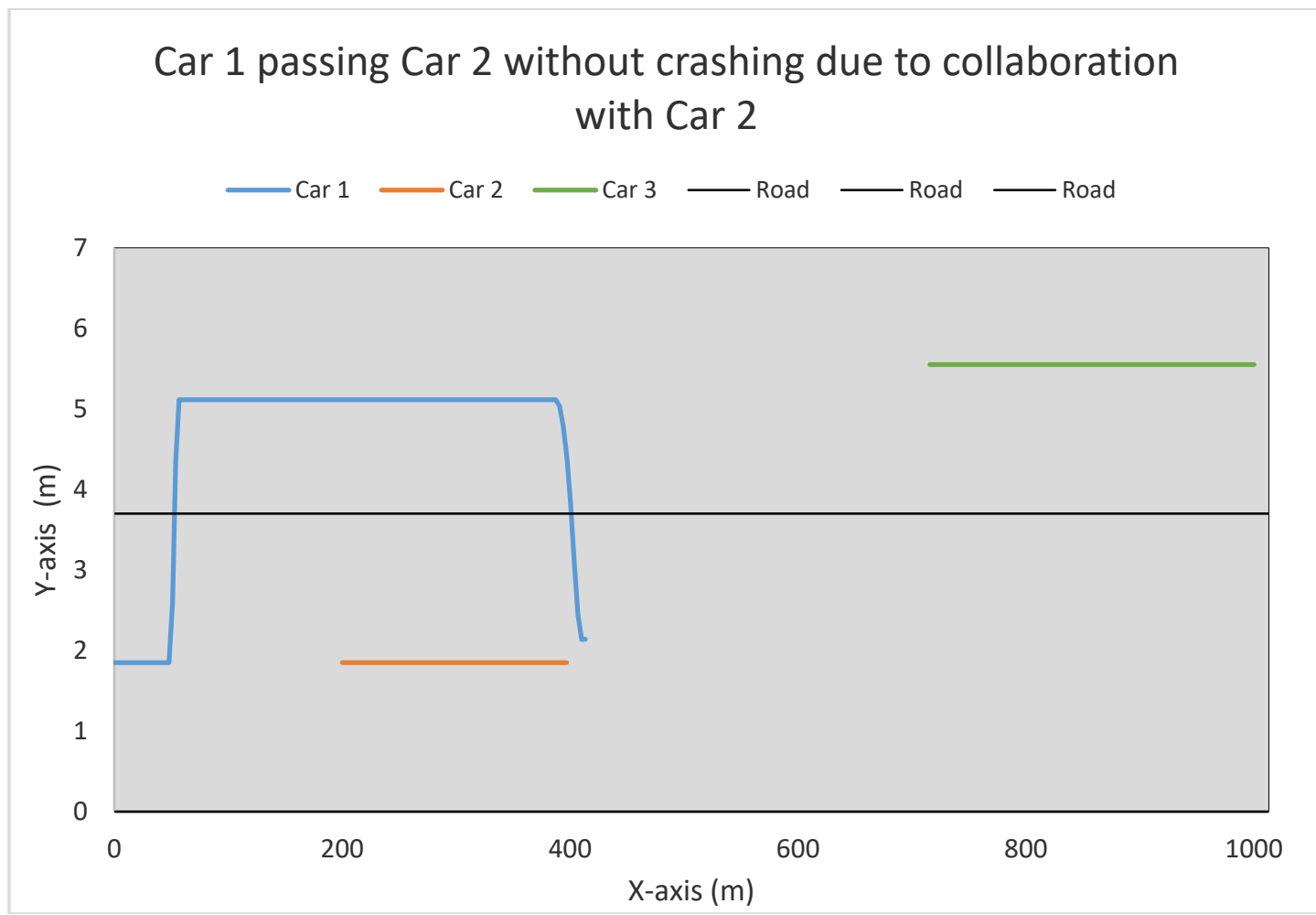

Figure 32: Car 1 passing Car 2 without crashing due to collaboration with Car 2 


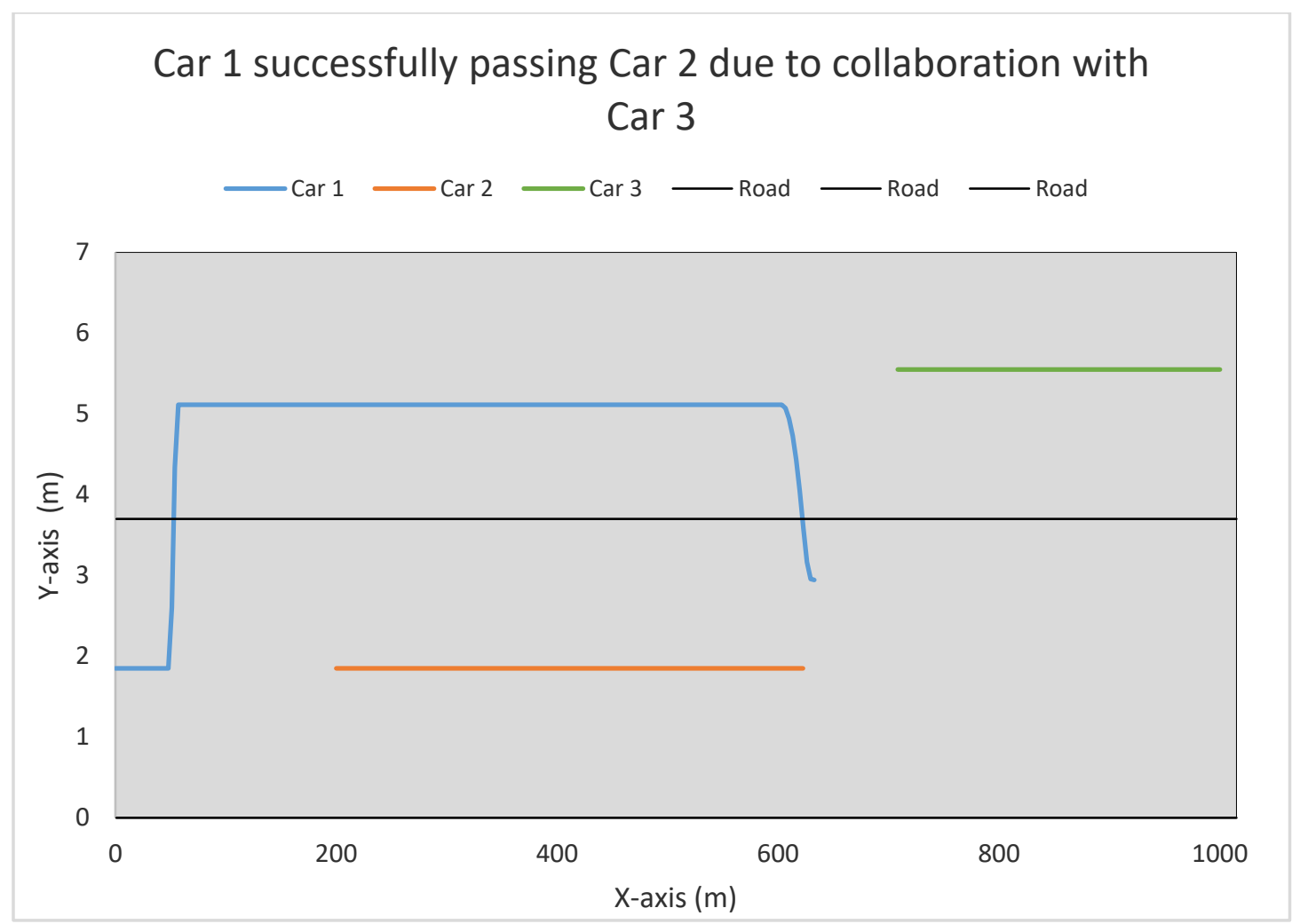

Figure 33: Car 1 successfully passing Car 2 due to collaboration with Car 3

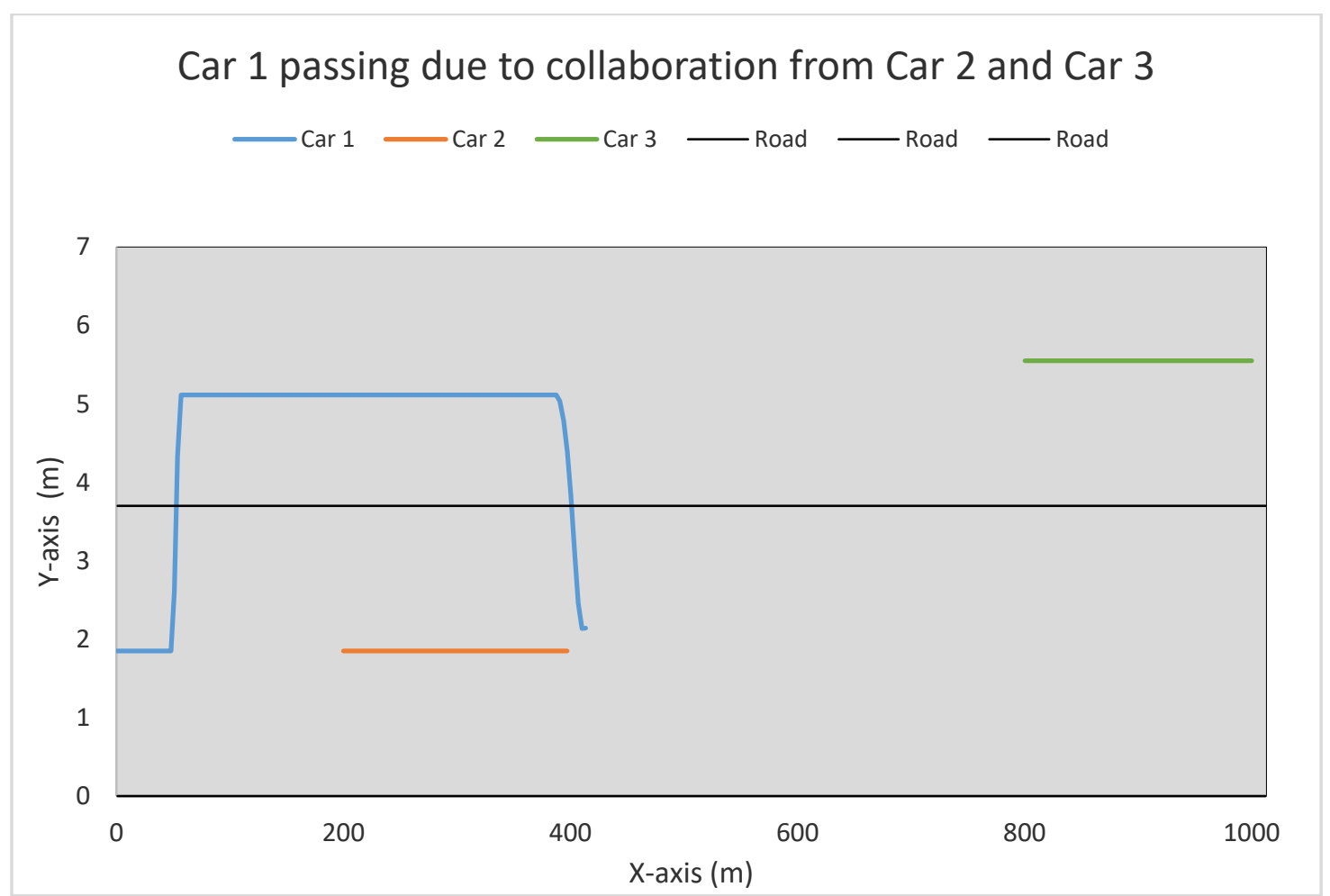

Figure 34: Car 1 passing due to collaboration from Car 2 and Car 3 
To study the effects of collaboration on five design factors, one design factor was varied at a time while keeping others fixed at nominal values. 1000 iterations were done. For the cases where car 1 decided to pass, they were segregated into 2 classes where both cars 2 and 3 collaborated, and the case where either 1 of the cars collaborated or none of the cars collaborated. For each class, count of crashes was counted and divided by total count of instances in that class to get the ratio of crashed versus total count of attempts to pass. This ratio is used to study the effects of collaboration. As shown in Figure 35, collaboration significantly decreases the count of crashes for high uncertainty is position measurement. Similarly, as shown in Figure 36 and Figure 37, collaboration significantly decreases the count of crashes for high uncertainty is velocity and acceleration measurement too. This shows, collaboration among the three cars, can help to mitigate the uncertainty in measurements.

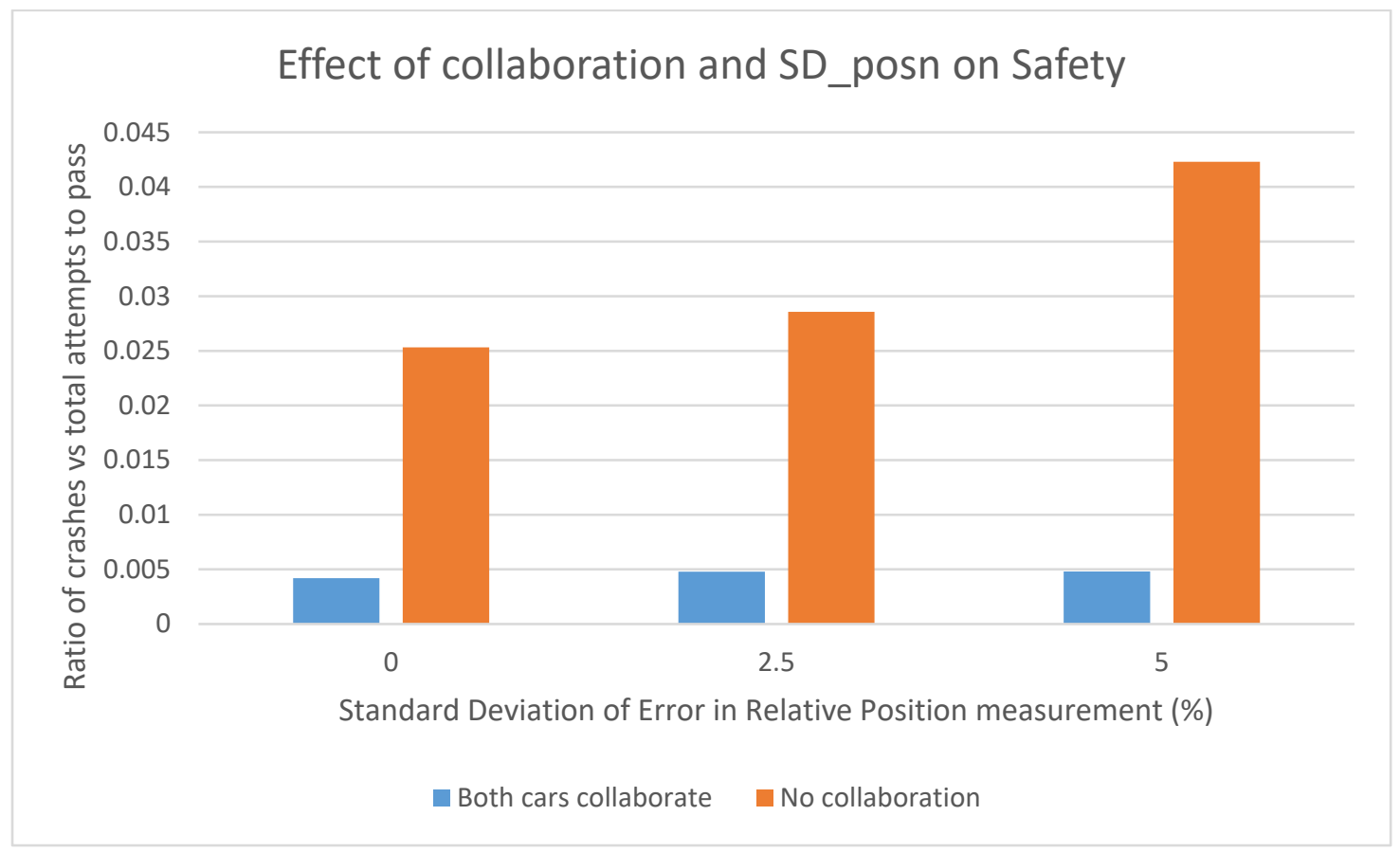

Figure 35: Effect of collaboration and Standard Deviation of Error in Relative Position measurement (\%) on Safety 


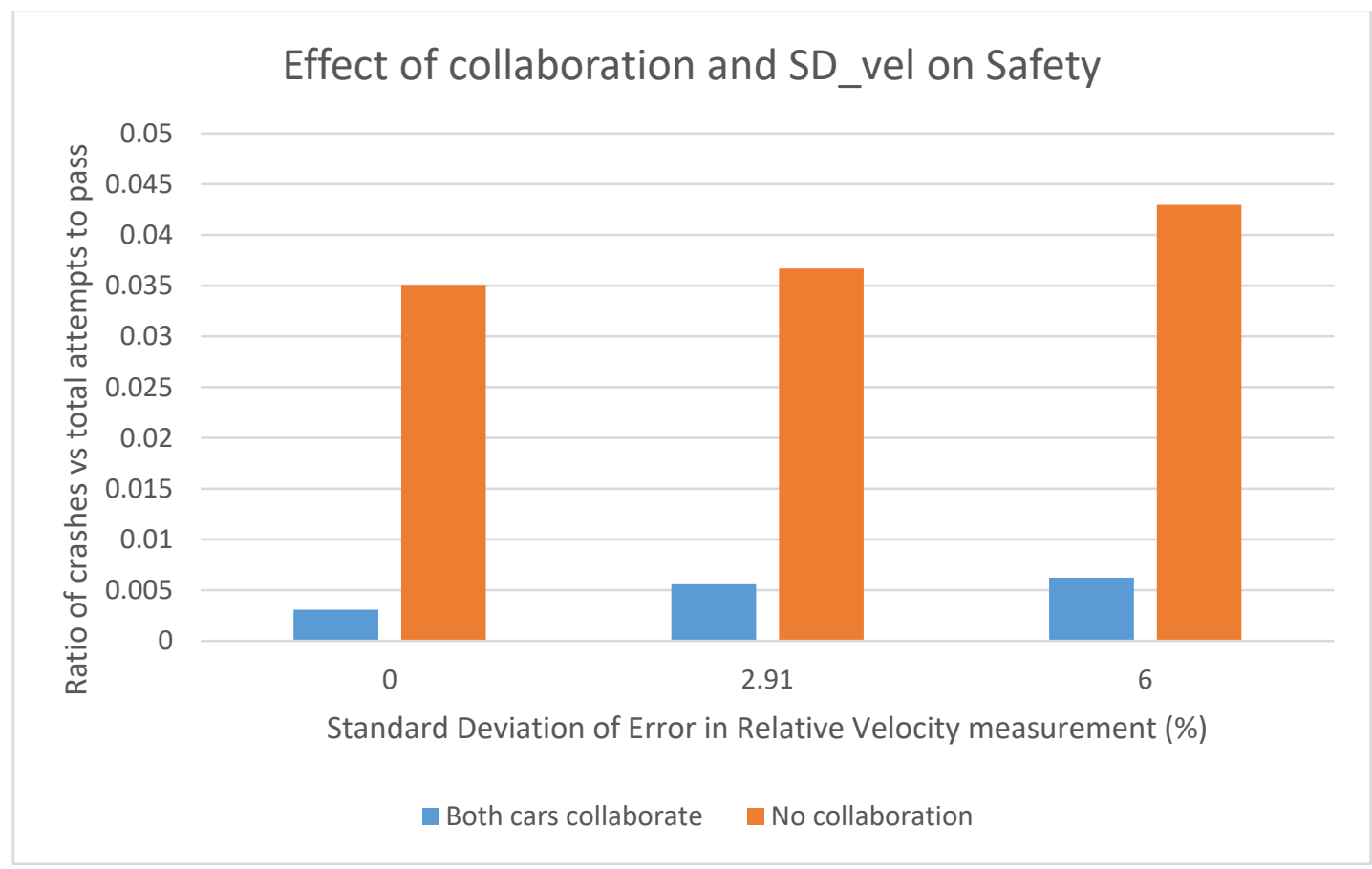

Figure 36: Effect of collaboration and Standard Deviation of Error in Relative Velocity measurement (\%) on Safety

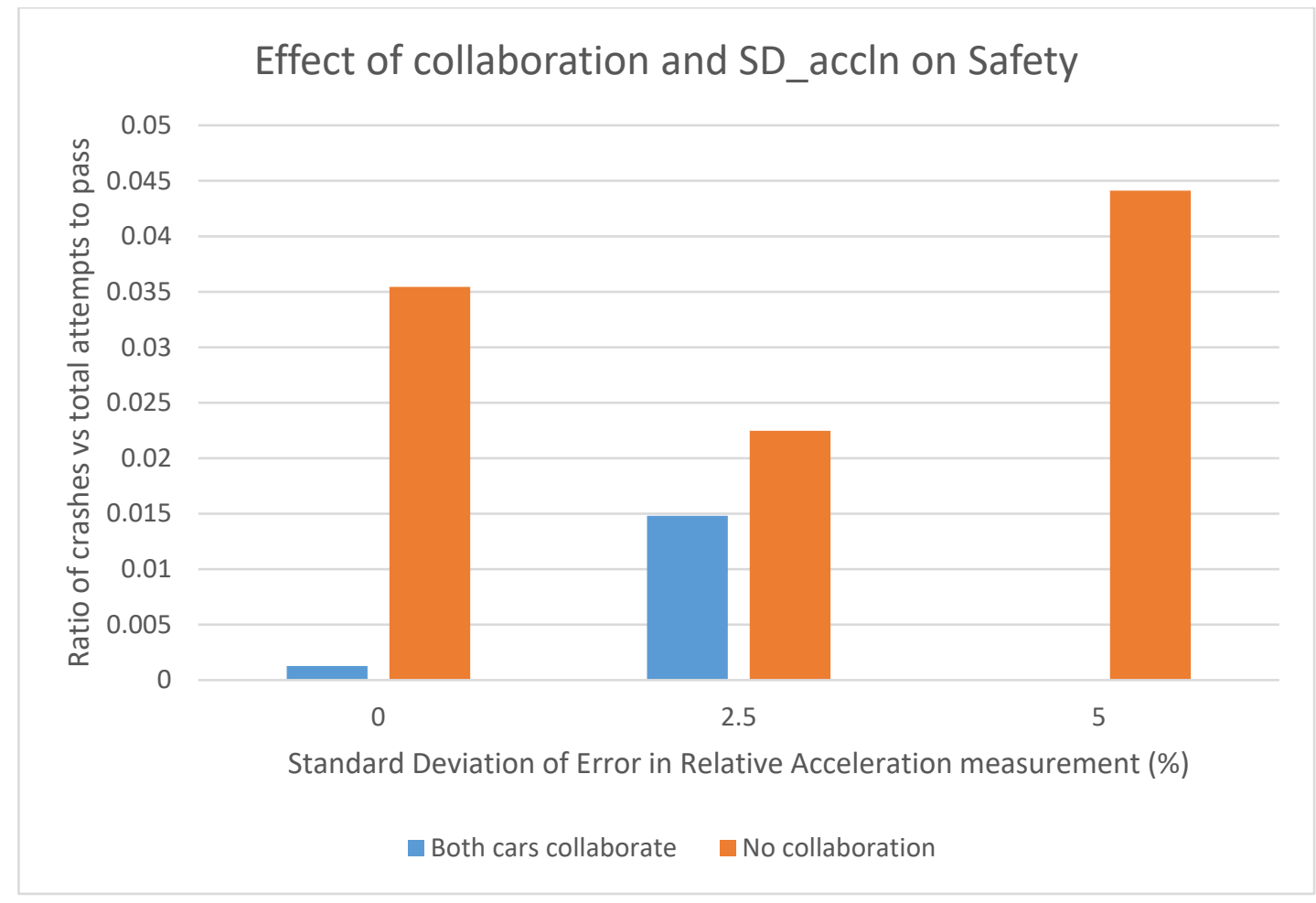

Figure 37: Effect of collaboration and Standard Deviation of Error in Relative Acceleration measurement (\%) on Safety 
Effect of collaboration on safety with respect to clearance distance is shown in Figure 38 and Figure 39. With increase in clearance distance and collaboration among the cars, there is no crash.

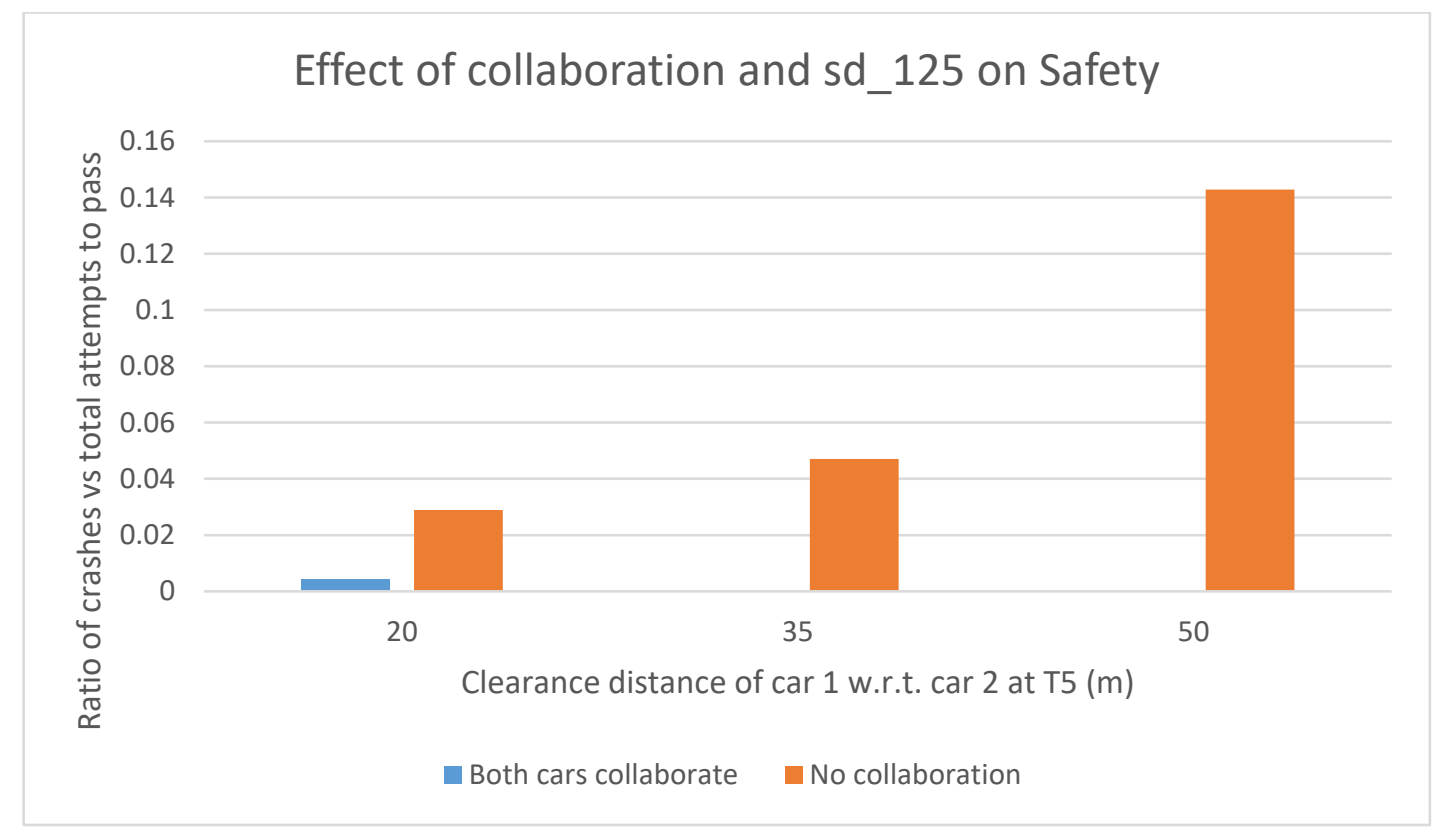

Figure 38: Effect of collaboration and Clearance distance of car 1 w.r.t. car 2 at T5 on Safety

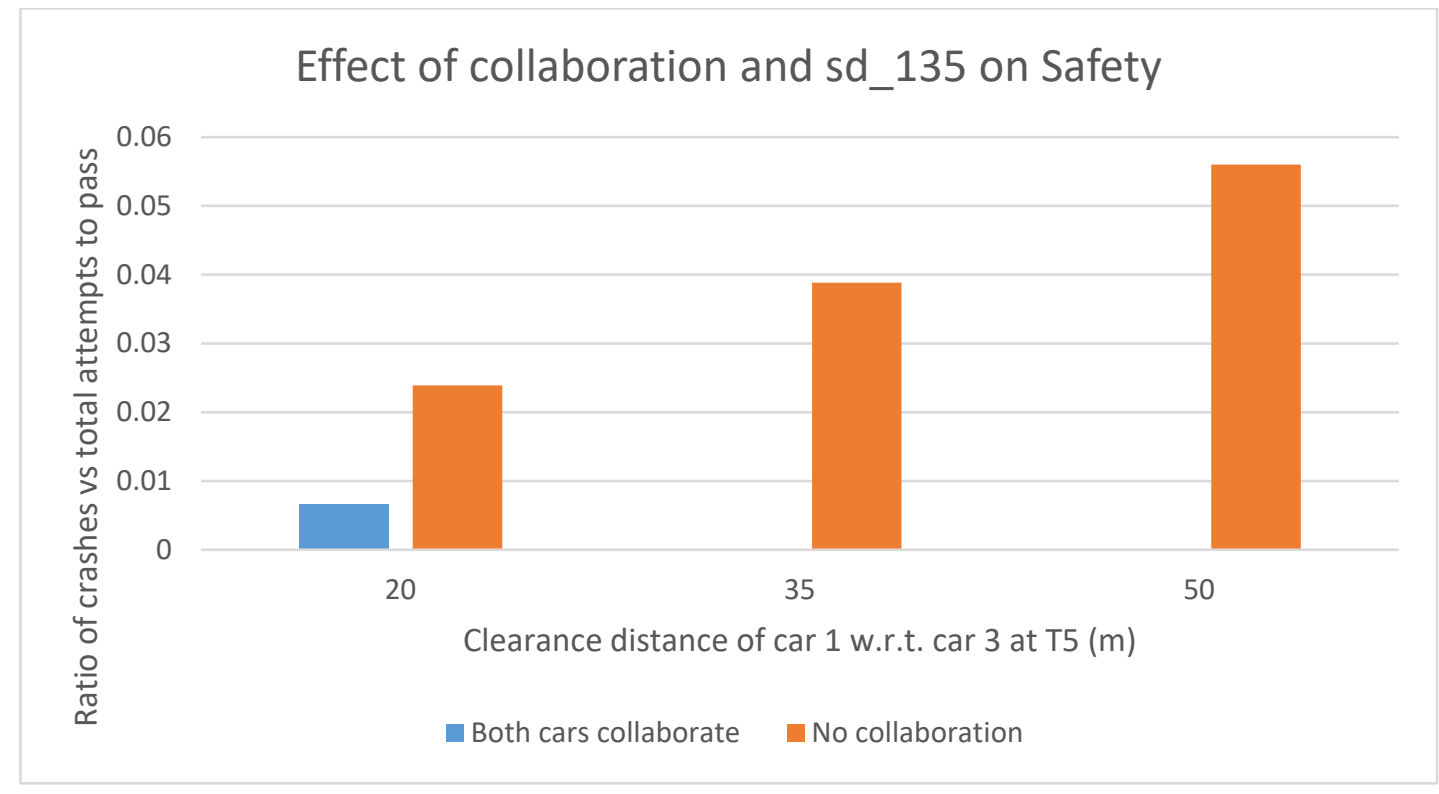

Figure 39: Effect of collaboration and Clearance distance of car 1 w.r.t. car 3 at T5 on Safety 


\subsection{Sensitivity Analysis}

To observe sensitivity of ' $\mathrm{SD}_{\text {posn', ' }} \mathrm{SD}_{\mathrm{vel}}$ ', 'SD $\mathrm{SD}_{\mathrm{accln}}$ ', 'sd $\mathrm{s}_{125}$ ' and 'sd $\mathrm{d}_{135}$ ' with respect to $\mathrm{P}(\mathrm{A}), \mathrm{P}(\mathrm{B})$ and $\mathrm{P}(\mathrm{C})$, all the three probabilities are calculated for each combination of maximum, minimum and nominal values of these design parameters by varying one parameter at a time, irrespective of collaborating amongst the cars. Collaboration factor is chosen randomly. Minimum value for ' $\mathrm{SD}_{\text {posn', ' }} \mathrm{D}_{\text {vel' }}$ ' and ' $S D_{\text {accln' }}$ ' is assumed to be $0 \%$. Nominal value for ' $\mathrm{SD}_{\text {posn' }}$ ' ' $\mathrm{SD}_{\text {vel }}$ ' and ' $\mathrm{SD}_{\text {accln }}$ ' is $2.5 \%$, $2.91 \%$ and $2.5 \%$ respectively. Maximum value for ' $S D_{\text {posn' }}$ ' ' $S D_{\text {vel' }}$ and ' $S D_{\text {accln }}$ ' is $5 \%$, $6 \%$ and 5\% respectively. Minimum value for ' $\mathrm{sd}_{125}$ ' and ' $\mathrm{sd}_{135}$ ' is assumed to be $20 \mathrm{~m}$. Nominal value for 'sd $\mathrm{d}_{125}$ ' and 'sd $\mathrm{d}_{135}$ ' is assumed to be $35 \mathrm{~m}$. Maximum value for ' $\mathrm{sd}_{125}$ ' and ' $\mathrm{sd}_{135}$ ' is assumed to be $50 \mathrm{~m}$. This gives rise to 11 cases as shown in Table 4. For each case 400 iterations of safe and unsafe initial state were executed to calculate $\mathrm{P}(\mathrm{A})$, $\mathrm{P}(\mathrm{B})$ and $\mathrm{P}(\mathrm{C})$. Change in each design factors with respect to probabilities is shown in the graphs in this section followed by a tornado diagram for $\mathrm{P}(\mathrm{A})$.

Table 4: Cases for Sensitivity Analysis

\begin{tabular}{|r|r|r|r|r|r|}
\hline S. No. & \multicolumn{1}{|c|}{ SD_posn (\%) } & SD_vel (\%) & SD_accln (\%) & sd_125 (m) & sd_135 (m) \\
\hline 1 & $\mathbf{0}$ & 2.91 & 2.5 & 35 & 35 \\
\hline 2 & $\mathbf{5}$ & 2.91 & 2.5 & 35 & 35 \\
\hline 3 & 2.5 & $\mathbf{0}$ & 2.5 & 35 & 35 \\
\hline 4 & 2.5 & $\mathbf{6}$ & 2.5 & 35 & 35 \\
\hline 5 & 2.5 & 2.91 & $\mathbf{0}$ & 35 & 35 \\
\hline 6 & 2.5 & 2.91 & $\mathbf{5}$ & 35 & 35 \\
\hline 7 & 2.5 & 2.91 & 2.5 & $\mathbf{2 0}$ & 35 \\
\hline 8 & 2.5 & 2.91 & 2.5 & $\mathbf{5 0}$ & 35 \\
\hline 9 & 2.5 & 2.91 & 2.5 & 35 & $\mathbf{2 0}$ \\
\hline 10 & 2.5 & 2.91 & 2.5 & 35 & $\mathbf{5 0}$ \\
\hline 11 & 2.5 & 2.91 & 2.5 & 35 & 35 \\
\hline
\end{tabular}




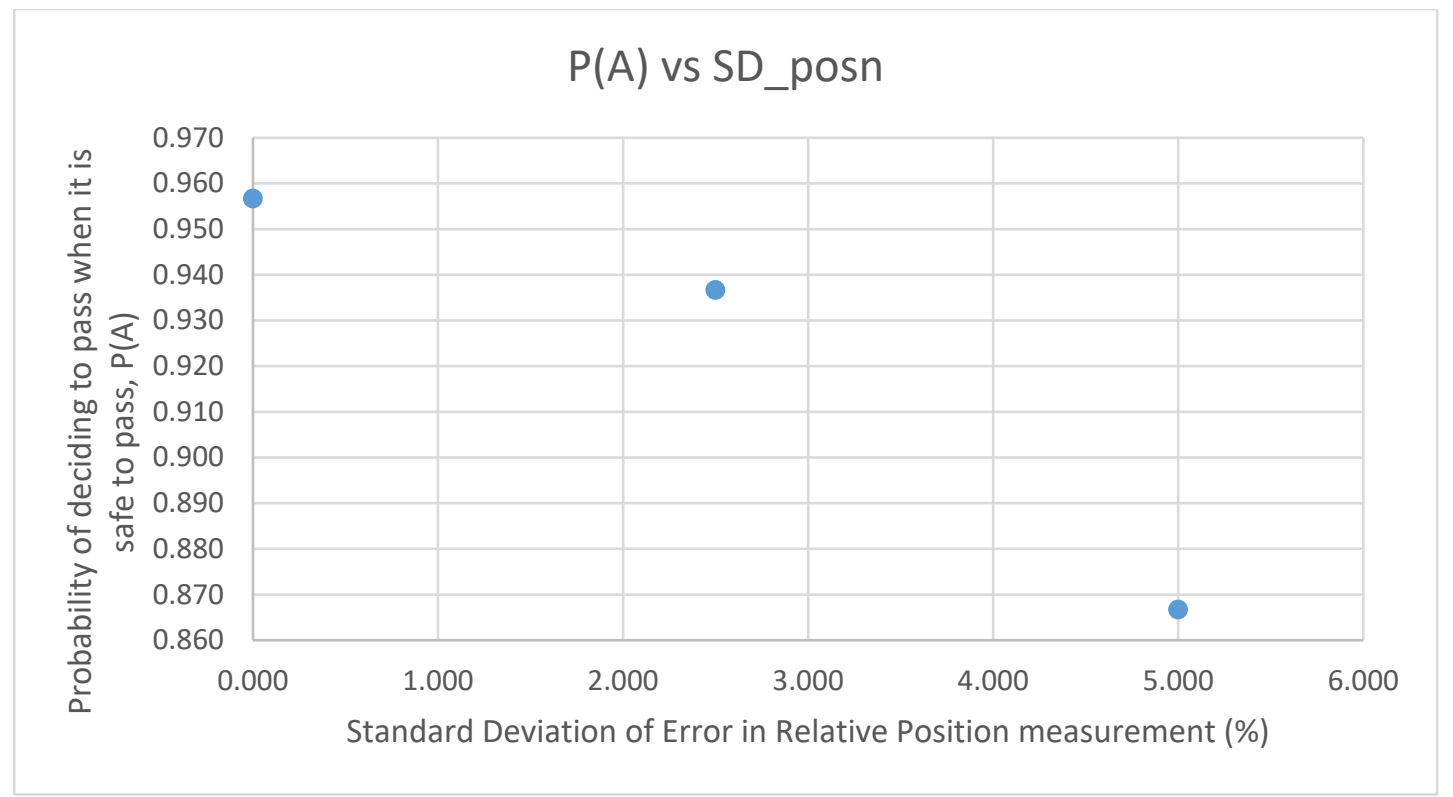

Figure 40: Probability of deciding to pass when it is safe to pass vs Standard deviation of error in Relative Position measurement

Figure 40 shows that increase in uncertainty in position measurement decreases probability of deciding to pass given that it is safe to pass. Figure 41 shows that increase in uncertainty in velocity measurement decreases probability of deciding to pass given that it is safe to pass.

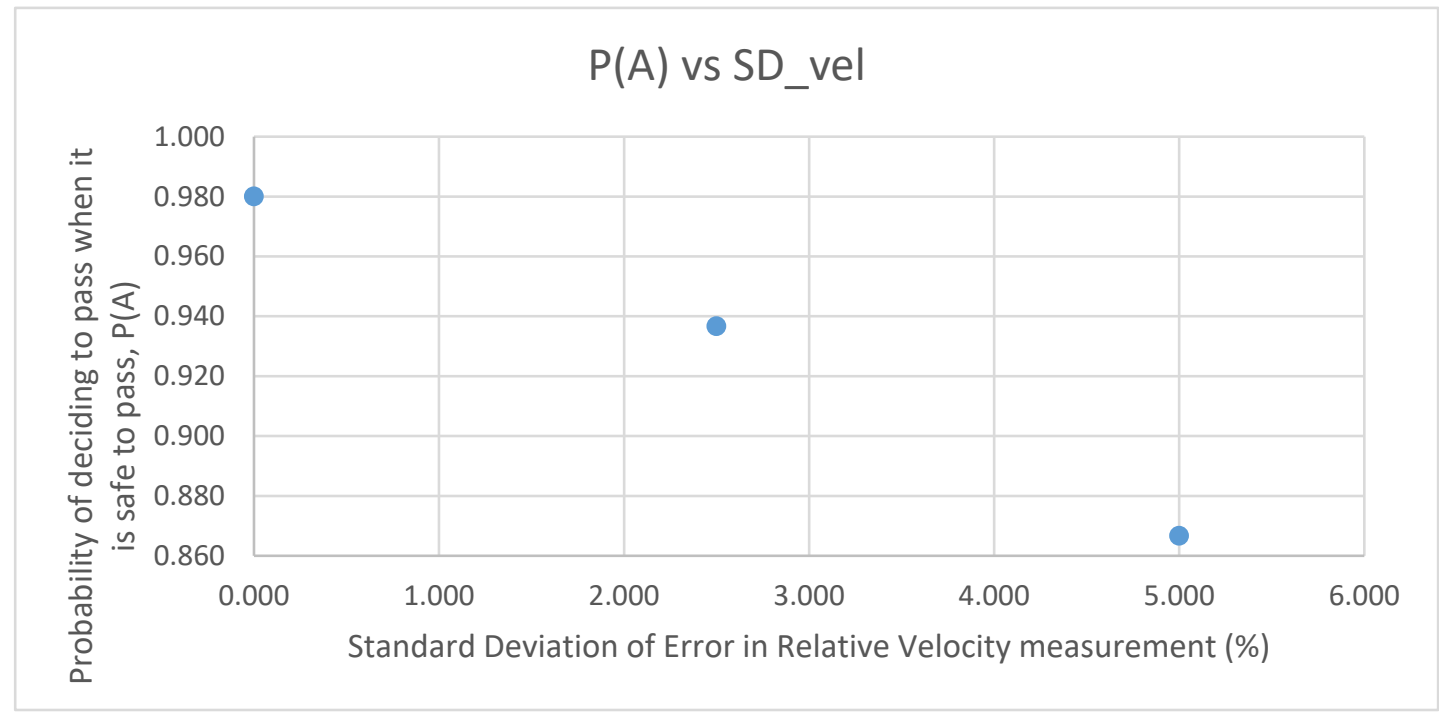

Figure 41: Probability of deciding to pass when it is safe to pass vs Standard deviation of error in Relative Velocity measurement 


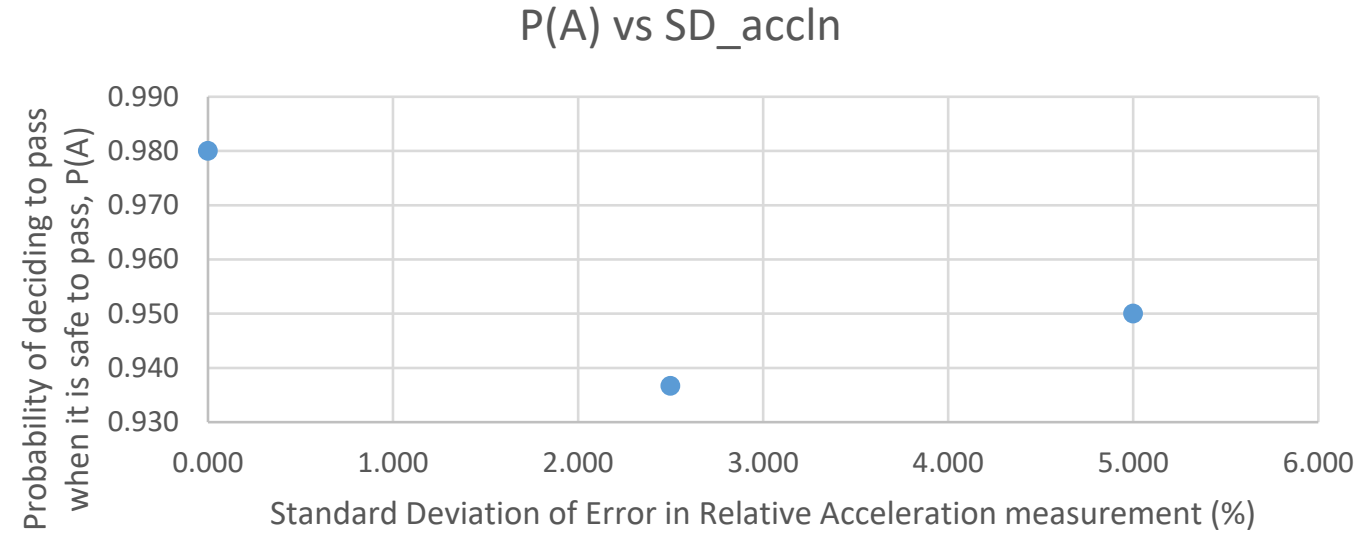

Figure 42: Probability of deciding to pass when it is safe to pass vs Standard deviation of error in Relative Acceleration measurement

Figure 42 shows that probability of deciding to pass given that it is safe to pass is minimum at the nominal value and increases if uncertainty increases or decreases from the nominal value. This observation needs further investigation. Figure 43 shows that increase in clearance distance of car 1 w.r.t. to car 2 at T5 used to make decision whether to execute the passing maneuver, decreases probability of deciding to pass given that it is safe to pass.

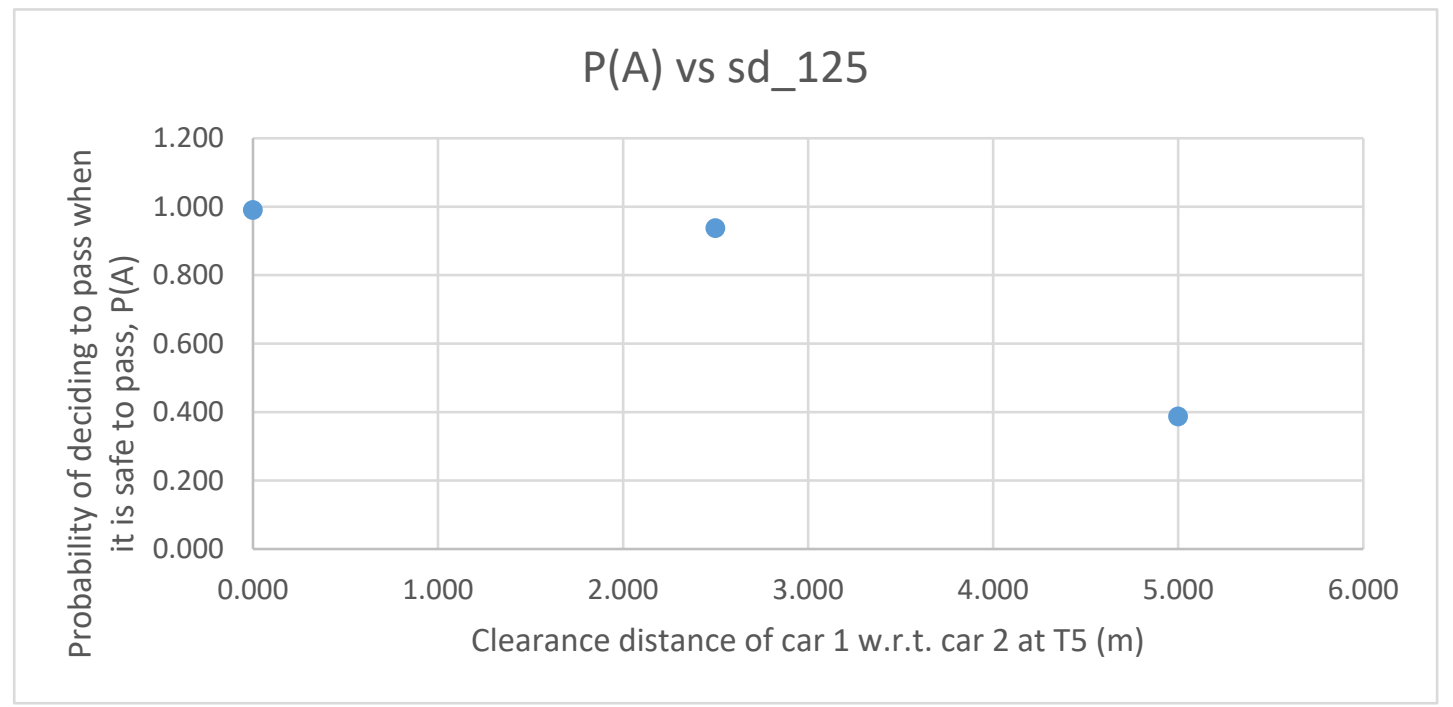

Figure 43: Probability of deciding to pass when it is safe to pass vs clearance distance of car 1 w.r.t. car 2 at $T 5$ 


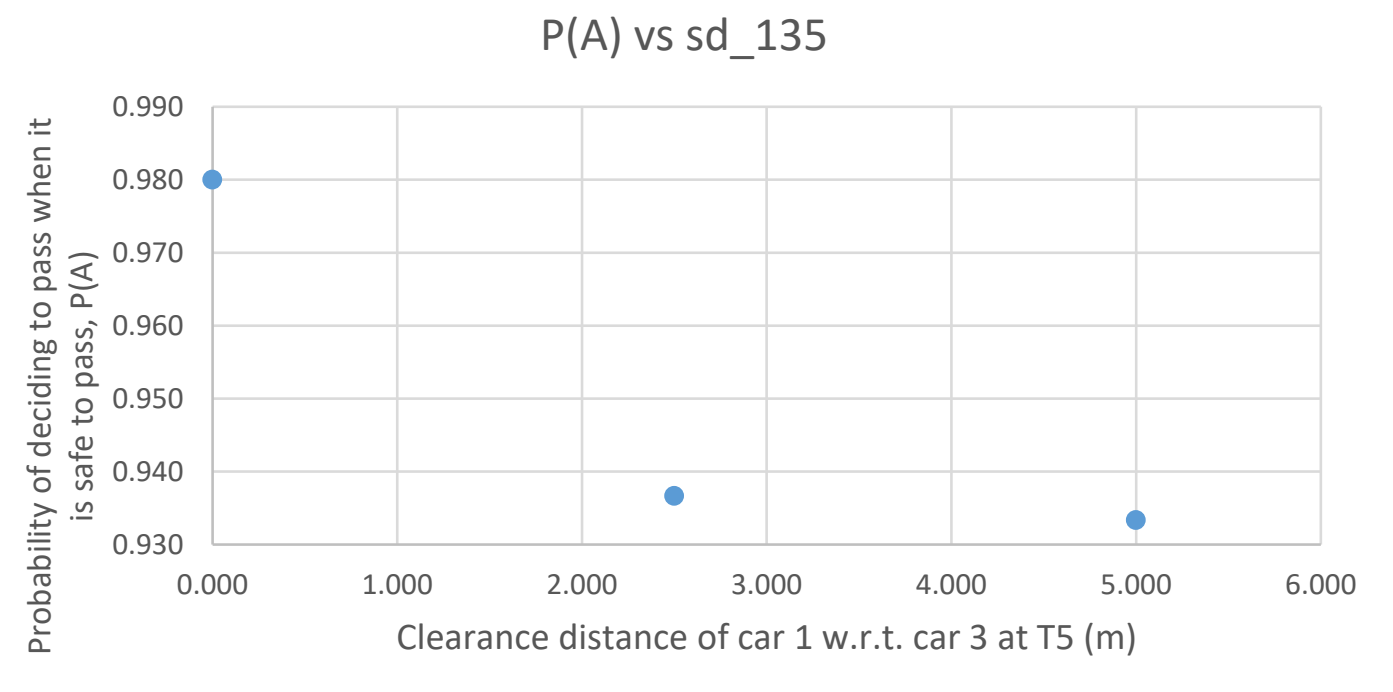

Figure 44: Probability of deciding to pass when it is safe to pass vs clearance distance of car 1 w.r.t. car 3 at $T 5$

Figure 44 shows that increase in clearance distance of car 1 w.r.t. to car 3 at T5 used to make decision whether to execute the passing maneuver, decreases probability of deciding to pass given that it is safe to pass. Figure 45 shows that increase in uncertainty in position measurement decreases probability of not deciding to pass given that it is unsafe to pass.

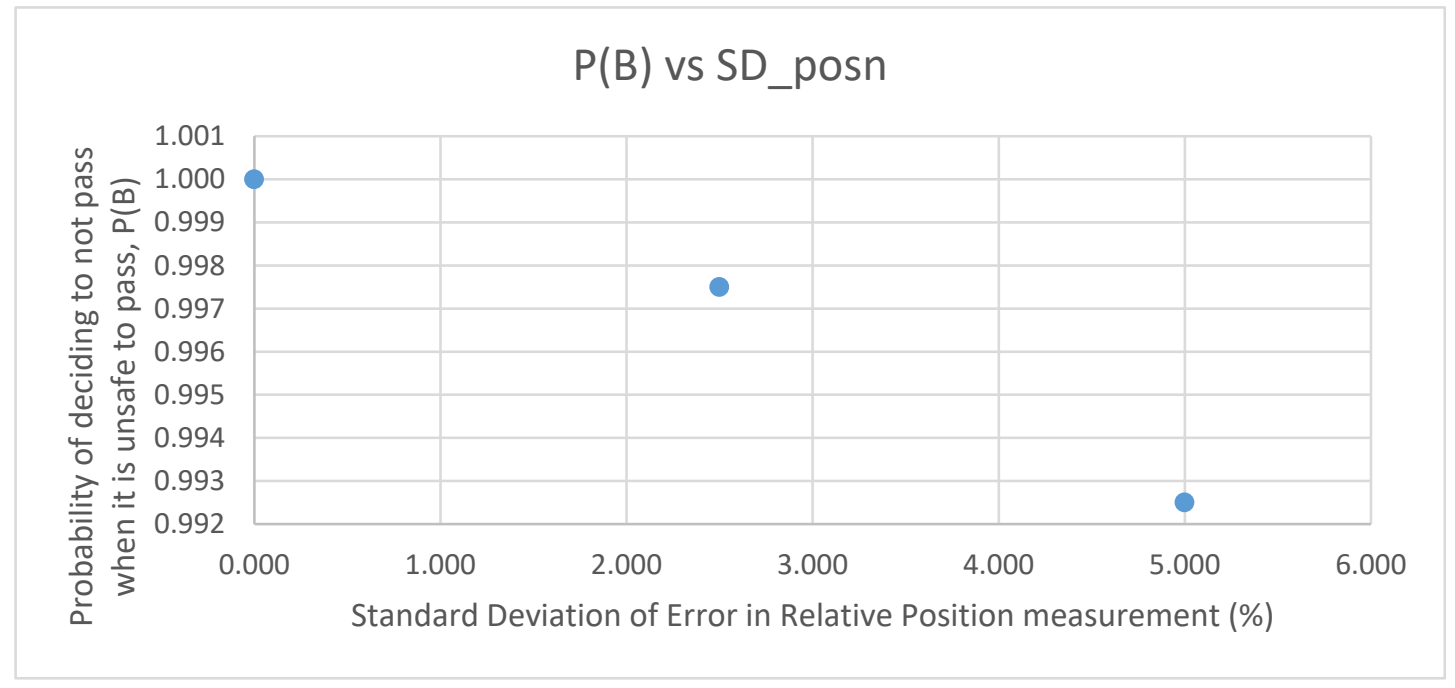

Figure 45: Probability of deciding not to pass when it is unsafe to pass vs Standard Deviation of error in Relative Position Measurement 


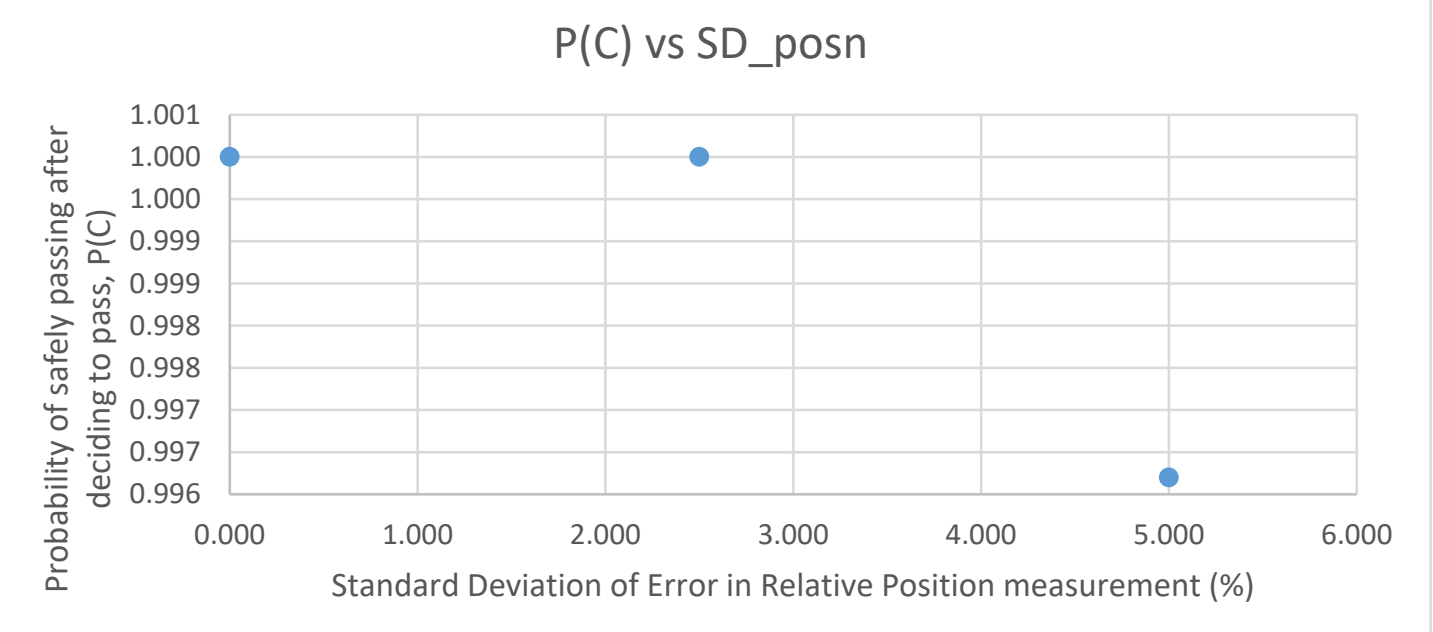

Figure 46: Probability of safely passing after deciding to pass vs Standard deviation of error in Relative Position measurement

Figure 46 shows that increase in uncertainty in position measurement, decreases probability of safely completing the passing maneuver after deciding to pass. Figure 47 shows that increase in clearance distance of car 1 w.r.t. car 2 at T5 used to make the decision whether to pass, increases the probability of not deciding to pass given that it is unsafe to pass, till the nominal value of clearance distance and any further increase in clearance distance decreases the probability $\mathrm{P}(\mathrm{B})$. This is an interesting observation as an increase in clearance distance should indicate higher chances of car 1 deciding to not pass. Thus, this requires further investigation.

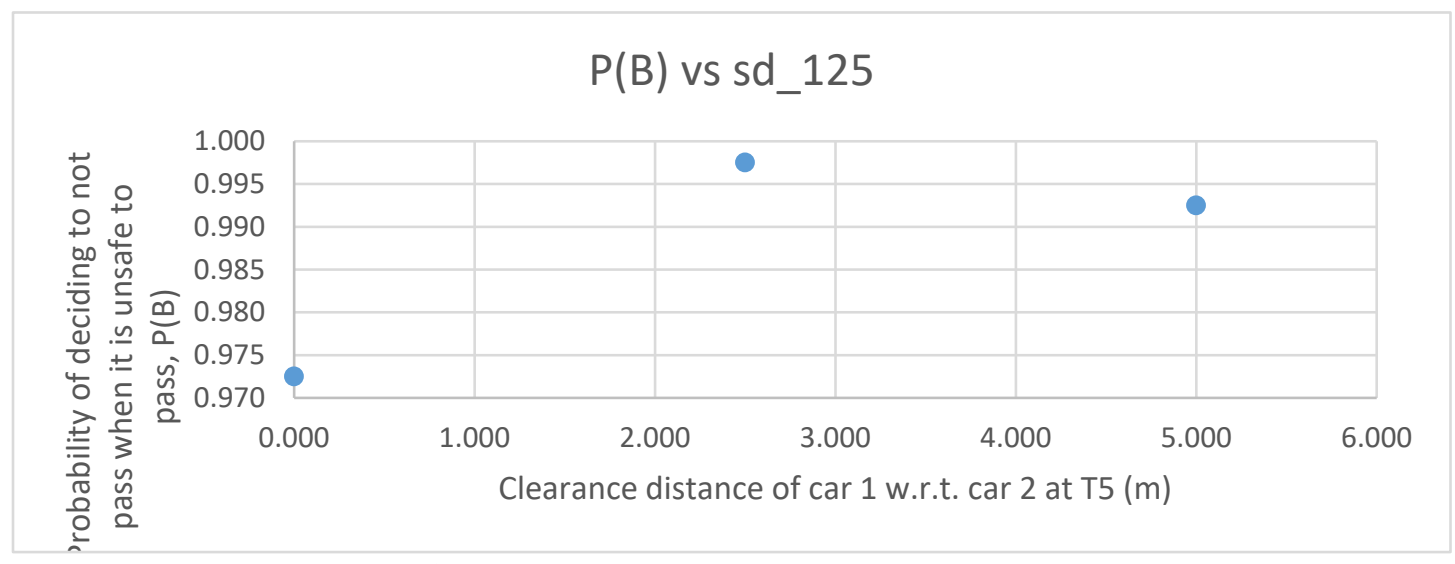

Figure 47: Probability of deciding not to pass when it is unsafe to pass vs clearance distance of car 1 w.r.t. car 2 at $T 5$ 


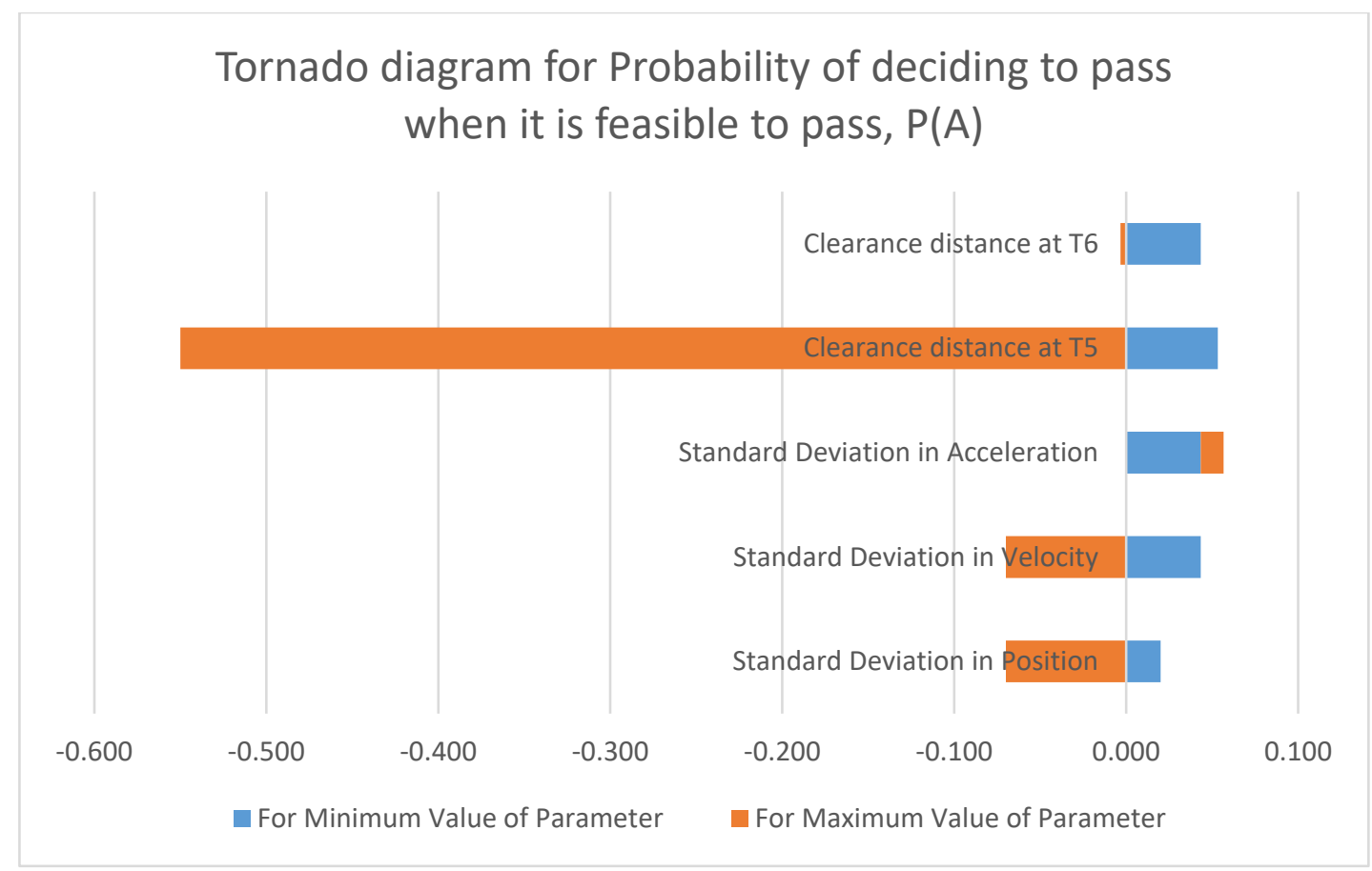

Figure 48: Sensitivity Analysis of $\mathrm{P}(\mathrm{A})$

From the tornado diagram, Figure 48, $\mathrm{P}(\mathrm{A})$ is most sensitive to 'sd125', 'SD posn' and ' $\mathrm{SD}_{\text {vel' }}$ ' with decrease in any of these increases $\mathrm{P}(\mathrm{A})$. For $\mathrm{P}(\mathrm{B})$ and $\mathrm{P}(\mathrm{C})$, change in the value of the probabilities was of the order of $10^{-3}$ and $10^{-4}$ respectively, which are less than the standard error for associated experiments.

\subsection{Trade-off Analysis}

For conducting trade-off analysis, 2 designs options for each position measurement system, velocity measurement system and acceleration measurement system were selected as described below. For position measurement, standard deviation of error is 5\% for monocular visual odometry and 2.5\% for Universal Medium Range Radar sensor. Standard deviation of error in measurement of velocity is $6 \%$ for vision-based constraint optical flow and $2.91 \%$ for Ultrasonic sensor system based on two- 
dimensional state method. Standard deviation of error in measurement of acceleration is assumed to be either $5 \%$ or $2.5 \%$. Further, clearance distance of car 1 w.r.t. car 2 and 3 at T5 can take three values $-20 \mathrm{~m}, 35 \mathrm{~m}$ and $50 \mathrm{~m}$. This gives rise to 72 test cases. For each case, 400 iterations of the model were executed separately for safe and unsafe initial state, followed by calculation of $\mathrm{P}(\mathrm{A}), \mathrm{P}(\mathrm{B})$ and $\mathrm{P}(\mathrm{C})$ as shown in Table 5.

Table 5: Different Design options for Car Passing Maneuver

\begin{tabular}{|c|c|c|c|c|c|c|c|c|}
\hline $\begin{array}{l}\text { S. } \\
\text { No. }\end{array}$ & $\begin{array}{c}\text { SD_posn } \\
\text { (\%) }\end{array}$ & $\begin{array}{c}\text { SD_vel } \\
\text { (\%) }\end{array}$ & $\begin{array}{c}\text { SD_accln } \\
(\%)\end{array}$ & $\begin{array}{c}\text { sd_125 } \\
\text { (m) }\end{array}$ & $\begin{array}{c}\text { sd_135 } \\
\text { (m) }\end{array}$ & $P(A)$ & $P(B)$ & $P(C)$ \\
\hline 1 & 2.5 & 2.91 & 2.5 & 20 & 20 & 1.000 & 0.96 & 0.98 \\
\hline 2 & 2.5 & 2.91 & 2.5 & 20 & 35 & 0.990 & 0.96 & 1.00 \\
\hline 3 & 2.5 & 2.91 & 2.5 & 20 & 50 & 0.990 & 0.99 & 1.00 \\
\hline 4 & 2.5 & 2.91 & 2.5 & 35 & 20 & 0.980 & 0.99 & 1.00 \\
\hline 5 & 2.5 & 2.91 & 2.5 & 35 & 35 & 0.920 & 0.99 & 1.00 \\
\hline 6 & 2.5 & 2.91 & 2.5 & 35 & 50 & 0.930 & 0.99 & 1.00 \\
\hline 7 & 2.5 & 2.91 & 2.5 & 50 & 20 & 0.590 & 0.99 & 1.00 \\
\hline 8 & 2.5 & 2.91 & 2.5 & 50 & 35 & 0.460 & 0.99 & 1.00 \\
\hline 9 & 2.5 & 2.91 & 2.5 & 50 & 50 & 0.240 & 1.00 & 1.00 \\
\hline 10 & 2.5 & 2.91 & 5 & 20 & 20 & 0.980 & 0.97 & 1.00 \\
\hline 11 & 2.5 & 2.91 & 5 & 20 & 35 & 1.000 & 0.99 & 1.00 \\
\hline 12 & 2.5 & 2.91 & 5 & 20 & 50 & 0.960 & 0.98 & 1.00 \\
\hline 13 & 2.5 & 2.91 & 5 & 35 & 20 & 0.920 & 1.00 & 1.00 \\
\hline 14 & 2.5 & 2.91 & 5 & 35 & 35 & 0.950 & 1.00 & 1.00 \\
\hline 15 & 2.5 & 2.91 & 5 & 35 & 50 & 0.920 & 1.00 & 1.00 \\
\hline 16 & 2.5 & 2.91 & 5 & 50 & 20 & 0.490 & 1.00 & 1.00 \\
\hline 17 & 2.5 & 2.91 & 5 & 50 & 35 & 0.290 & 1.00 & 1.00 \\
\hline 18 & 2.5 & 2.91 & 5 & 50 & 50 & 0.170 & 1.00 & 1.00 \\
\hline 19 & 2.5 & 6 & 2.5 & 20 & 20 & 0.980 & 0.90 & 1.00 \\
\hline 20 & 2.5 & 6 & 2.5 & 20 & 35 & 0.980 & 0.97 & 1.00 \\
\hline 21 & 2.5 & 6 & 2.5 & 20 & 50 & 0.970 & 0.99 & 1.00 \\
\hline 22 & 2.5 & 6 & 2.5 & 35 & 20 & 0.910 & 0.99 & 1.00 \\
\hline 23 & 2.5 & 6 & 2.5 & 35 & 35 & 0.860 & 1.00 & 1.00 \\
\hline 24 & 2.5 & 6 & 2.5 & 35 & 50 & 0.810 & 0.99 & 1.00 \\
\hline 25 & 2.5 & 6 & 2.5 & 50 & 20 & 0.500 & 0.99 & 1.00 \\
\hline
\end{tabular}




\begin{tabular}{|c|c|c|c|c|c|c|c|c|}
\hline $\begin{array}{l}\text { S. } \\
\text { No. }\end{array}$ & $\begin{array}{c}\text { SD_posn } \\
\text { (\%) }\end{array}$ & $\begin{array}{c}\text { SD_vel } \\
\text { (\%) }\end{array}$ & $\begin{array}{c}\text { SD_accln } \\
(\%)\end{array}$ & $\begin{array}{c}\text { sd_125 } \\
\text { (m) }\end{array}$ & $\begin{array}{c}\text { sd_135 } \\
\text { (m) }\end{array}$ & $P(A)$ & $\mathrm{P}(\mathrm{B})$ & $P(C)$ \\
\hline 26 & 2.5 & 6 & 2.5 & 50 & 35 & 0.360 & 1.00 & 1.00 \\
\hline 27 & 2.5 & 6 & 2.5 & 50 & 50 & 0.200 & 1.00 & 1.00 \\
\hline 28 & 2.5 & 6 & 5 & 20 & 20 & 1.000 & 0.93 & 1.00 \\
\hline 29 & 2.5 & 6 & 5 & 20 & 35 & 0.990 & 0.95 & 1.00 \\
\hline 30 & 2.5 & 6 & 5 & 20 & 50 & 0.960 & 0.98 & 0.99 \\
\hline 31 & 2.5 & 6 & 5 & 35 & 20 & 0.930 & 0.99 & 1.00 \\
\hline 32 & 2.5 & 6 & 5 & 35 & 35 & 0.830 & 1.00 & 1.00 \\
\hline 33 & 2.5 & 6 & 5 & 35 & 50 & 0.770 & 1.00 & 1.00 \\
\hline 34 & 2.5 & 6 & 5 & 50 & 20 & 0.430 & 1.00 & 1.00 \\
\hline 35 & 2.5 & 6 & 5 & 50 & 35 & 0.350 & 0.99 & 1.00 \\
\hline 36 & 2.5 & 6 & 5 & 50 & 50 & 0.270 & 1.00 & 1.00 \\
\hline 37 & 5 & 2.91 & 2.5 & 20 & 20 & 0.960 & 0.93 & 1.00 \\
\hline 38 & 5 & 2.91 & 2.5 & 20 & 35 & 0.990 & 0.95 & 1.00 \\
\hline 39 & 5 & 2.91 & 2.5 & 20 & 50 & 0.950 & 0.97 & 1.00 \\
\hline 40 & 5 & 2.91 & 2.5 & 35 & 20 & 0.880 & 0.995 & 1.00 \\
\hline 41 & 5 & 2.91 & 2.5 & 35 & 35 & 0.840 & 0.985 & 1.00 \\
\hline 42 & 5 & 2.91 & 2.5 & 35 & 50 & 0.850 & 1.000 & 1.00 \\
\hline 43 & 5 & 2.91 & 2.5 & 50 & 20 & 0.370 & 1.000 & 1.00 \\
\hline 44 & 5 & 2.91 & 2.5 & 50 & 35 & 0.280 & 1.000 & 1.00 \\
\hline 45 & 5 & 2.91 & 2.5 & 50 & 50 & 0.170 & 1.000 & 1.00 \\
\hline 46 & 5 & 2.91 & 5 & 20 & 20 & 0.970 & 0.94 & 1.00 \\
\hline 47 & 5 & 2.91 & 5 & 20 & 35 & 0.970 & 0.93 & 0.99 \\
\hline 48 & 5 & 2.91 & 5 & 20 & 50 & 0.990 & 0.98 & 1.00 \\
\hline 49 & 5 & 2.91 & 5 & 35 & 20 & 0.910 & 1.00 & 1.00 \\
\hline 50 & 5 & 2.91 & 5 & 35 & 35 & 0.890 & 1.00 & 1.00 \\
\hline 51 & 5 & 2.91 & 5 & 35 & 50 & 0.750 & 0.99 & 1.00 \\
\hline 52 & 5 & 2.91 & 5 & 50 & 20 & 0.410 & 1.00 & 1.00 \\
\hline 53 & 5 & 2.91 & 5 & 50 & 35 & 0.240 & 0.99 & 1.00 \\
\hline 54 & 5 & 2.91 & 5 & 50 & 50 & 0.250 & 1.00 & 1.00 \\
\hline 55 & 5 & 6 & 2.5 & 20 & 20 & 0.990 & 0.88 & 0.98 \\
\hline 56 & 5 & 6 & 2.5 & 20 & 35 & 0.950 & 0.94 & 1.00 \\
\hline 57 & 5 & 6 & 2.5 & 20 & 50 & 0.920 & 0.99 & 1.00 \\
\hline 58 & 5 & 6 & 2.5 & 35 & 20 & 0.800 & 0.99 & 1.00 \\
\hline 59 & 5 & 6 & 2.5 & 35 & 35 & 0.780 & 0.99 & 1.00 \\
\hline 60 & 5 & 6 & 2.5 & 35 & 50 & 0.770 & 0.99 & 1.00 \\
\hline 61 & 5 & 6 & 2.5 & 50 & 20 & 0.490 & 0.99 & 1.00 \\
\hline 62 & 5 & 6 & 2.5 & 50 & 35 & 0.320 & 0.99 & 1.00 \\
\hline 63 & 5 & 6 & 2.5 & 50 & 50 & 0.270 & 0.99 & 1.00 \\
\hline 64 & 5 & 6 & 5 & 20 & 20 & 0.980 & 0.90 & 1.00 \\
\hline 65 & 5 & 6 & 5 & 20 & 35 & 0.960 & 0.94 & 1.00 \\
\hline 66 & 5 & 6 & 5 & 20 & 50 & 0.950 & 0.97 & 1.00 \\
\hline
\end{tabular}




\begin{tabular}{|c|r|r|r|r|r|r|r|r|}
\hline $\begin{array}{c}\text { S. } \\
\text { No. }\end{array}$ & $\begin{array}{c}\text { SD_posn } \\
\mathbf{( \% )}\end{array}$ & \multicolumn{1}{c|}{$\begin{array}{c}\text { SD_vel } \\
\mathbf{( \% )}\end{array}$} & $\begin{array}{c}\text { SD_accln } \\
\mathbf{( \% )}\end{array}$ & $\begin{array}{c}\text { sd_125 } \\
\mathbf{( m )}\end{array}$ & $\begin{array}{c}\text { sd_135 } \\
\mathbf{( m )}\end{array}$ & \multicolumn{1}{c|}{$\mathbf{P ( A )}$} & \multicolumn{1}{c|}{$\mathbf{P ( B )}$} & P(C) \\
\hline 67 & 5 & 6 & 5 & 35 & 20 & 0.870 & 0.99 & 0.99 \\
\hline 68 & 5 & 6 & 5 & 35 & 35 & 0.770 & 1.00 & 1.00 \\
\hline 69 & 5 & 6 & 5 & 35 & 50 & 0.620 & 1.00 & 0.98 \\
\hline 70 & 5 & 6 & 5 & 50 & 20 & 0.450 & 1.00 & 1.00 \\
\hline 71 & 5 & 6 & 5 & 50 & 35 & 0.300 & 1.00 & 1.00 \\
\hline 72 & 5 & 6 & 5 & 50 & 50 & 0.230 & 1.00 & 1.00 \\
\hline
\end{tabular}

From the 72 test cases, solutions were filtered by identifying the Pareto dominating solutions. This reduced the number of solutions to 2 solutions shown in Table 6. For position measurement, standard deviation of error is $2.5 \%$, i.e., Universal Medium Range Radar sensor. Standard deviation of error in measurement of velocity is $2.91 \%$, i.e., Ultrasonic sensor system based on two-dimensional state method. Standard deviation of error in measurement of acceleration is 5\%. Further, clearance distance of car 1 w.r.t. car 3 at T5 is 35m. Clearance distance of car 1 w.r.t. car 2 at T5 can be $20 \mathrm{~m}$ or $35 \mathrm{~m}$, depending on choice of design and importance of the three metrics.

Table 6: Pareto Dominant Design options for Car Overtaking Maneuver

\begin{tabular}{|r|r|r|r|r|r|r|r|r|}
\hline S. No. & $\begin{array}{c}\text { SD_posn } \\
\text { (\%) }\end{array}$ & $\begin{array}{c}\text { SD_vel } \\
\mathbf{( \% )}\end{array}$ & $\begin{array}{c}\text { SD_accln } \\
\mathbf{( \% )}\end{array}$ & $\begin{array}{c}\text { sd_125 } \\
\mathbf{( m )}\end{array}$ & $\begin{array}{c}\text { sd_135 } \\
\mathbf{( m )}\end{array}$ & $\mathbf{P ( A )}$ & \multicolumn{1}{c|}{$\mathbf{P ( B )}$} & \multicolumn{1}{c|}{$\mathbf{P ( C )}$} \\
\hline 11 & 2.5 & 2.91 & 5 & 20 & 35 & 1.00 & 0.99 & 1.00 \\
\hline 14 & 2.5 & 2.91 & 5 & 35 & 35 & 0.95 & 1.00 & 1.00 \\
\hline
\end{tabular}

\subsection{Results}

From the analysis of the data collected using simulations, it is observed that:

- To safely pass car 2, autonomous car should reach maximum allowed velocity as soon as possible. 
- Angular orientation of car 1 at T2 and T5 is observed to be 20 degrees and 20 degrees, as determined by the controller. It can be deduced that car 1 should have smaller angle with respect to $\mathrm{x}$-axis while turning.

- Total mean turning time for the maneuver is 16.6 seconds which is comparable real life Passing maneuver execution. The trajectory taken by the controller corresponds to flying Passing.

- For cases with unsafe initial state, it was observed that if both car 2 and car 3 do not cooperate, it always results in crash.

- $\mathrm{P}(\mathrm{A})$ is most sensitive to 'sd $\mathrm{d}_{125}$ ', ' $\mathrm{S} \mathrm{D}_{\text {posn }}$ ' and ' $\mathrm{SD}_{\text {vel' }}$ '.

- 2 dominant solutions were found with Universal Medium Range Radar sensor, Ultrasonic sensor system based on two-dimensional state method, standard deviation of error in measurement of acceleration is $5 \%$, clearance distance of car 1 w.r.t. car 3 at T5 is 35m and clearance distance of car 1 w.r.t. car 2 at T5 can be $20 \mathrm{~m}$ or $35 \mathrm{~m}$. 


\section{Chapter 10: Conclusions and Future Work}

In this work, a functional architecture of the car passing problem has been modelled using rigorous Model-based Systems Engineering to analyze the complex problem. Based on the architecture of the problem, especially behavior identified through state machines, controller for the three cars were modelled. As each component of the car passing problem has clearly defined interfaces and functionality in the model, it can be replaced by models of different design options for that component to analyze system performance. To demonstrate this, a mathematical model of different components, including controller for car 1, was developed in MATLAB, and performance of the system in terms of making the correct decision whether to pass and safety of the passing maneuver if it is executed, was evaluated. Further, some design variables were identified from the model, and different design options were chosen for those design variables. This was followed by a sensitivity analysis and tradeoff analysis based on the performance of the system for different combination of the design options. Different courses of action were narrowed down to two choices using Pareto analysis. The work demonstrates how MBSE approach facilitates managing complexity, making system modular while allowing design space exploration and evaluation.

The results presented are based on analysis of two initial states and one scenario from amongst many scenarios that an autonomous vehicle may face on the road, like moving back after deciding to execute the passing maneuver. Trends obtained from the analysis should hold true in general, but many more factors need to be considered to generate data that will accurately and precisely represent the real-life scenarios. The 
human behavior modelling done in the model is just an approximation of the human behaviors of interest to the analysis and doesn't account for all the quirks of human behavior. Analysis depends highly on the type of distribution of measurement error and its mean and standard deviation. Better results can be obtained by conducting human factors studies to relate cooperativeness of the drivers towards other vehicles passing them and how they react to the situation.

Next step will be to formally verify and validate the simulation. Higher fidelity models for different component like measurement systems and car actuator models can be used to consider factors like actuation delay, measurement delay and processing speed of the controller. These higher fidelity models can be used to evaluate performance of different design combinations using simulations and derive requirements for the components. One must always consider that the car passing problem is critical to human safety and MBSE approach will help towards developing safer and reliable systems. 


\section{Bibliography}

[1] A. H. Ghods and F. . F. Saccomanno, "Development and evaluation of a microscopic overtaking gap acceptance model for two-lane highways," Canadian Journal of Civil Engineering, pp. 573-582, 2016.

[2] C. Liu and Z. Wang, "Passing Segment Length Determination on Two-Lane Highways," Transportation Research Procedia, vol. 25, pp. 491-496, 2017.

[3] M. Bahram, C. Hubmann , A. Lawitzky and . M. Aeberhard, "A Combined Model- and Learning-Based Framework for Interaction-Aware Maneuver Prediction," IEEE Transactions on Intelligent Transportation Systems, vol. 17, no. 6, pp. 1538-1550, 2016.

[4] G. Mwesige, H. Farah, U. Bagampadde and H. N. Koutsopoulos, "A Model and Its Applications for Predicting Passing Rate at Passing Zones on Two-Lane Rural Highways," Journal of Transportation Engineering, vol. 142, no. 3, 2016.

[5] A. Mihály, Z. Á. Milacski, Z. Tösér, A. Lörincz, T. Tettamanti and I. Varga, "Cost and risk sensitive decision making and control for highway overtaking maneuver," in Models and Technologies for Intelligent Transportation Systems (MT-ITS), Budapest, Hungary, 2015.

[6] A. D. Jarnea, R. Dobrescu, D. Popescu and L. Ichim, "Advanced driver assistance system for overtaking maneuver on a highway," in System Theory, Control and Computing (ICSTCC), Cheile Gradistei, Romania, 2015.

[7] R. Chen, K. Kusano and H. Gabler, "Driver Behavior During Overtaking Maneuvers from the 100-Car Naturalistic Driving Study," vol. 16, p. S176\{S181, 2015.

[8] M. Motro, A. Chu, J. Choi and R. W. Heath, "Vehicular ad-hoc network simulations of overtaking maneuvers on two-lane rural highways," Transportation Research Part C Emerging Technologies, pp. 72-60, 2016.

[9] C. Haskins, K. Forsberg, M. Krueger, D. Walden and D. Hamelin, Systems Engineering Handbook, San Diego, CA: INCOSE, 2011.

[10] I. C. o. S. E. (INCOSE), Systems Engineering, September 2007.

[11] J. S. Baras, Key Slides for the Model-based Systems Engineering, 2018.

[12] L. Caihong, W. Fengying, S. Yong, L. Zhenying and W. Zhi, "A Complete Coverage Path Planning Algorithm for Mobile Robot Based on FSM and Rolling Window Approach in Unknown Environment," in Chinese Control Conference, 2015.

[13] C. Katrakazas, M. Quddus, W.-H. Chen and L. Deka, "Real-time motion planning methods for autonomous on-road driving: State-of-the-art and future research directions," 2015. 
[14] M. Naveed, . D. Kitchin and A. Crampton, "Monte-Carlo Planning for Pathfinding in Real-Time Strategy Games," 2010.

[15] "CE 4dr Sedan," Autoblog, [Online]. Available: https://www.autoblog.com/buy/2007-Toyota-Camry-CE_4dr_Sedan/specs/.

[16] A. Kell, "Engineer Explains: Lidar," 5 April 2019. [Online]. Available: https://blog.cometlabs.io/engineer-explains-lidar-748f9ba0c404.

[17] G. Johansson and K. Rumar, "Drivers' Brake Reaction Times," The Journal of the Human Factors and Ergonomics Society, 1972.

[18] "Interstate Highway standards," Wikipedia, [Online]. Available: https://en.wikipedia.org/wiki/Interstate_Highway_standards.

[19] M. Strauss and J. Carnahan, "Observed Errors in Distance Estimates," in SAE Special Publication, Detroit, MI, 2010.

[20] S. K. Rushton and P. A. Duke, "Observers cannot accurately estimate the speed of an," Vision Research, vol. 49, no. 15, pp. 1919-1928, 2009.

[21] M. O. A. Aqel, M. H. Marhaban, M. I. Saripan and N. . B. Ismail, "Review of visual odometry: types, approaches, challenges, and," Springerplus, 28 October 2016.

[22] F. d. P. Müller, "Survey on Ranging Sensors and Cooperative Techniques for Relative Positioning of Vehicles," MDPI, 31 January 2017.

[23] M. Jalalat, M. Nejati and A. Majidi, "Vehicle Detection and Speed Estimation Using Cascade Classifier and Sub-pixel Stereo Matching," in Signal Processing and Intelligent Systems (ICSPIS), Tehran, Iran, 2017.

[24] J. Liu, J. Han, H. Lv and B. Li, "An Ultrasonic Sensor System Based on a TwoDimensional State Method for Highway Vehicle Violation Detection Applications," Sensors, 2015.

[25] V. N. Gadepally, "Estimation of Driver Behavior for Autonomous Vehicle," 2013.

[26] C. Llorca, A. T. Moreno, A. Lenorzer, J. Casas and A. Garcia, "Development of a new microscopic passing maneuver model for two-lane rural roads," Transportation Research Part C: Emerging Technologies, vol. 52, no. March 2015, pp. 152-172, 2015.

[27] A. T. Moreno, C. Llorca, A. García and A. Lenorzer, "Design Criteria for Minimum Passing Zone Lengths," Transportation Research Record Journal of the Transportation, pp. 19-27, 2015. 